\title{
LOW MOMENTUM TRANSFER MEASUREMENTS OF PION ELECTROPRODUCTION AND VIRTUAL COMPTON SCATTERING AT THE DELTA RESONANCE
}

\author{
A Dissertation \\ Submitted to \\ the Temple University Graduate Board
}

In Partial Fulfillment

of the Requirements for the Degree

DOCTOR OF PHILOSOPHY

by

Adam Blomberg

December 2016

Examining Committee Members:

Nikolaos Sparveris, Department of Physics, Advisory chair

Zein-Eddine Meziani, Department of Physics

Andreas Metz, Department of Physics

Alexandre Camsonne, External Member, Jefferson Laboratory 


\section{ABSTRACT}

Non-spherical components of the nucleon wave function are measured through $\mathrm{p}\left(e, e^{\prime} p\right) \pi^{\circ}$ experiment at the $\Delta^{+}(1232)$ resonance for $Q^{2}=0.04,0.09$, and $0.13(\mathrm{GeV} / \mathrm{c})^{2}$ utilizing the Jefferson National Accelerator Facility (JLab) pulsed beam and Hall A spectrometers. The new data extend the measurements of the Coulomb quadrupole amplitude to the lowest momentum transfer ever reached. The results disagree with predictions of constituent quark models and are in reasonable agreement with dynamical calculations that include pion cloud effects, chiral effective field theory and lattice calculations. The reported measurements indicate that improvement is required to the theoretical calculations and provide valuable input that will allow their refinements.

The Coulomb to magnetic multipole ratio (CMR) and generalized polarizability (GP) of the nucleon are also measured through virtual Compton scattering (VCS) for $Q^{2}=0.2$ $(\mathrm{GeV} / \mathrm{c})^{2}$ utilizing the Mainz Microtron (MAMI) continuous beam and A1 spectrometers. This data represents the first low $\mathrm{Q}^{2}$ GP measurement at the $\Delta^{+}(1232)$ resonance. The GP measurement explores a region where previous data and theoretical calculations disagree. The CMR measurement will be the first VCS extraction to compare with world data generated through pion electroproduction. The Dispersion Relation (DR) model used for the VCS extraction provides a new theoretical framework for the data signal and backgrounds that is largely independent from the pion electroproduction models. The independence of the DR from the traditional models provides a strong crosscheck on the ability of the models to isolate the data signal. 


\section{CONTENTS}

ABSTRACT

LIST OF FIGURES $\quad$ v

LIST OF TABLES X xiii

1 INTRODUCTION 1

2 THEORY 7

2.1 Kinematics . . . . . . . . . . . . . . . . . 7

2.2 Cross Sections . . . . . . . . . . . . . . . . . 10

2.3 Multipoles ........................... 11

2.4 Polarizability ............................ 14

2.5 Models ............................. 16

2.5.1 Pion Electroproduction . . . . . . . . . . . . 16

2.5.2 Virtual Compton Scattering . . . . . . . . . . . . 17

2.6 Measurements . . . . . . . . . . . . . . . . . . . 18

2.6.1 Pion Electroproduction . . . . . . . . . . . . . . . 18

2.6.2 Virtual Compton Scattering . . . . . . . . . . . . 20

2.7 Result Extraction . . . . . . . . . . . . . . . . . . . 20

3 EQUIPMENT $\quad 21$

3.1 Jefferson $\mathrm{Lab} \ldots \ldots \ldots \ldots \ldots \ldots$

3.1 .1 The Electron Beam . . . . . . . . . . . . . . . . 21

3.1.1.1 The Beamline . . . . . . . . . . . . . . 21

3.1.1.2 Beam Position and Direction . . . . . . . . . . 22

3.1.1.3 Beam Energy . . . . . . . . . . . . 22 
3.1.1.4 Beam Current . . . . . . . . . . . . . . . 23

3.1.1.5 Beam Polarization . . . . . . . . . . . . . . . . 24

3.1 .2 The Targets . . . . . . . . . . . . . . . . . . 24

3.1 .3 The Detectors . . . . . . . . . . . . . . . . . . . . . 25

3.1.3.1 Drift Chambers . . . . . . . . . . . . . . 28

3.1.3.2 Scintillating Panels . . . . . . . . . . . . . . 28

3.1.3.3 Cherenkov Detector . . . . . . . . . . . . . . . . . 29

3.1.3.4 Calorimeters . . . . . . . . . . . . . . . . . 29

3.2 Mainz Microtron . . . . . . . . . . . . . . . . . . 30

3.2 .1 The Electron Beam . . . . . . . . . . . . . . . . . . . . 31

3.2.1.1 The Beamline . . . . . . . . . . . . . . . 31

3.2.1.2 Beam Position and Direction . . . . . . . . . . . 32

3.2.1.3 Beam Energy . . . . . . . . . . . . . . . 34

3.2.1.4 Beam Current . . . . . . . . . . . . . . . . . . 34

3.2.1.5 Beam Polarization . . . . . . . . . . . . . . . 34

3.2 .2 The Targets . . . . . . . . . . . . . . . . . . . . . 34

3.2 .3 The Detectors . . . . . . . . . . . . . . . . . . . 35

3.2.3.1 Drift Chambers . . . . . . . . . . . . . . 35

3.2.3.2 Scintillating Panels . . . . . . . . . . . . . 38

3.2.3.3 Cherenkov Detector . . . . . . . . . . . . . . 38

4 CAlibrations $\quad 40$

4.1 Beam Current . . . . . . . . . . . . . . . . . 40

4.2 Beam Position . . . . . . . . . . . . . . . . . . . . . 42

4.3 Vertical Drift Chambers . . . . . . . . . . . . . . . . . 44

4.4 Optics Matrix . . . . . . . . . . . . . . . . . . . . 45

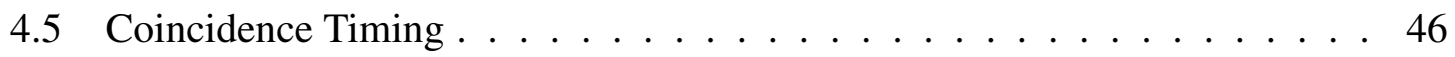

4.6 Calorimeters . . . . . . . . . . . . . . . . . . . . . . 49

4.7 Cherenkov Detectors . . . . . . . . . . . . . . . . 52

4.8 Scintillating Panels $\ldots \ldots \ldots \ldots \ldots \ldots \ldots \ldots$

5 DATA ANALYSIS $\quad 56$

5.1 Background subtraction . . . . . . . . . . . . . 56

5.2 Spectrometer Momentum . . . . . . . . . . . . . . . . . . . . 62

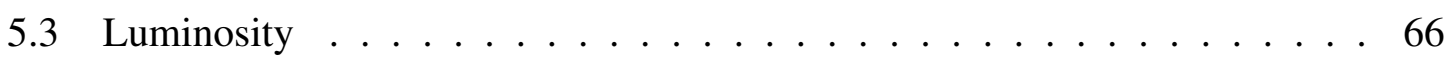


5.4 Proton absorption $\ldots \ldots \ldots \ldots \ldots \ldots \ldots$

5.5 Multiple Track Events . . . . . . . . . . . . . . . . . . . . . . . . 69

5.6 Efficiencies . . . . . . . . . . . . . . . . . . . 74

5.7 Phase space and Simulation . . . . . . . . . . . . . . 77

5.8 Bin centering $\ldots \ldots \ldots \ldots \ldots \ldots \ldots \ldots \ldots \ldots \ldots$

5.9 Fitting $\ldots \ldots \ldots \ldots \ldots \ldots \ldots \ldots \ldots \ldots \ldots \ldots \ldots \ldots$

5.10 Systematic uncertainties $\ldots \ldots \ldots \ldots \ldots$. . . . . . . . 82

6 RESULTS AND DISCUSSION $\quad 84$

6.1 JLab . . . . . . . . . . . . . . . . . . . . . . . . . . 84

6.1 .1 Cross Sections and Asymmetries . . . . . . . . . . . . . . 84

6.1 .2 Fitted Extractions . . . . . . . . . . . . . . . . . . . . 91

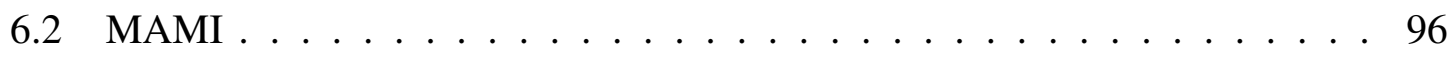

6.2.1 Cross Sections and Asymmetries . . . . . . . . . . . . . 96

6.2 .2 Fitted Extractions . . . . . . . . . . . . . . . . . . . . 98

$\begin{array}{lll}7 & \text { CONCLUSIONS } & 101\end{array}$

$\begin{array}{lc}\text { REFERENCES } & 103\end{array}$ 


\section{LIST OF FIGURES}

1.1 Figure from [30] demonstrating enhancement of pion cloud dynamics (shaded region) at low momentum transfer. . . . . . . . . . . . . . . . 2

1.2 World data and predictions for the multipole ratios in the low momentum transfer regime. Data from [13, 22, 24, 36, 37, 39] are shown with their statistical and systematic uncertainties added in quadrature. Theoretical predictions from MAID [32, 33], DMT [31], SAID [35], Sato-Lee [30], Capstick [8], HQM [49], the Lattice-QCD calculation [27], the ChEFT of Pascalutsa-Vanderhaegen (PV) [47] and the Gail-Hemmert (GH) [48] . . . 3

1.3 Simple model of proton describing its electric and magnetic polarizabilities. 4

1.4 World data [51-55] shown with open boxes were extracted with LEX, and open circles from DR. The solid curve corresponds to HBChPT [81, 82]. The dashed line shows dipole fall off from the DR $[60,61]$ calculation. . . . 5

2.1 Kinematics for pion electroproduction. Figure from [56]. . . . . . . . . 8

2.2 Kinematics for VCS. The scattering plane is defined by $\vec{k}_{i} \times \vec{k}^{\prime}$. The reaction

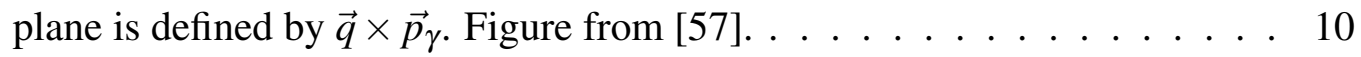

3.1 Overview of the JLab facility layout. Figure from [65]. . . . . . . . . 22

3.2 Target end cap view with beam position and raster shaded. Axes in mm. . . 23 
3.3 Overview of the Hall A beamline and beam monitors. Figure from [65]. . . 24

3.4 Diagram of HRS magnets. Figure from [65] . . . . . . . . . . . . 26

3.5 Overview of LHRS detector package. Figure from [65] . . . . . . . . . . . 27

3.6 Diagram of HRS magnets. Figure from [65]. . . . . . . . . . . . . 27

3.7 Diagram of VDC alignment within each HRS. Track reconstruction allows tagging of the data for particle momentum and number of particles entering the HRS. Figure from [65]. . . . . . . . . . . . . . . . . 28

3.8 Diagram of PID calorimeters in each HRS. Note the careful offsets to ensure no particles pass without traversing the detector. Figure from [65]. . . 30

3.9 Layout of the Mainz Microtron. The electron source and RTMs 1-3 are in the lower right, the HDSM is in the center, and experimental halls are to the right. Figure from [68]. . . . . . . . . . . . . . . 31

3.10 Overview of electron acceleration inside a RTM as seen at MAMI. Figure from [69]. . . . . . . . . . . . . . . . . . . . . . . 32

3.11 Overview of electron acceleration inside the HDSM at MAMI. Figure from [70] . . . . . . . . . . . . . . . . . . 33

3.12 Image of the rastered beam on BeO target taken Sept. 25, 2012. . . . . . . 33

3.13 Electromagnet layout of MAMI A1 Spectrometer A. Figure from [73] . . . 36

3.14 Electromagnet layout of MAMI A1 Spectrometer B. Figure from [73]. . . . 37

3.15 Detector package from A1 spectrometers. Figure from [71]. . . . . . . . . 39 
4.1 Calibration run of the beam current detectors near the CEBAF injection site. OL02 is an RF cavity current monitor. Figure from [58]. . . . . . . . . 41

4.2 Sample run showing voltage from four beam current monitors at JLab. fc and 0L02 (red and blue) are current monitors at the injector site, and the BCMs (green and yellow) are near the beamline entrance to Hall A. Figure from [58] . . . . . . . . . . . . . . . . . . . . . . 41

4.3 Top: Hall A BPM data from test runs with fixed beam positions (see labels). Bottom: CEBAF EPICS data from same runs, the different labeled positions show the need to calibrate the linear transformation matrix before using BPM data. Figure from [58]. . . . . . . . . . . . . . . . . 42

4.4 Test of the BPM calibration. The position read from the Hall A BPMs is plotted vs the EPICS positions. A linear fit shows good agreement after calibration. Figure from [58] . . . . . . . . . . . . . . . . . . . . 43

4.5 VDC wire timing spectrum before calibration. Figure from [58]. . . . . . . 44

4.6 VDC wire timing spectrum after calibration. Figure from [58]. . . . . . . . 45

4.7 Comparison of the $\mathrm{z}$ vertex reconstruction using the standard optics matrices, and the optimized matrices from Helene Fonveille. . . . . . . . . . . . 46

4.8 Coincidence timing plot from MAMI showing resolution comparable to design specifications. . . . . . . . . . . . . . . . . . 47

4.9 Double peak structure of the timing spectrum shown for each bar of the S2 scintillating plane. Bar 5, highlighted in red, shows the double peak from improper triggers. Figure from [58]. . . . . . . . . . . . . . . . . . 47 
4.10 Correcting the coincidence time of flight for correlations with spectrometer variables. Shown is the ILHRS momentum vs coincidence time before (left) and after (right) a timing correction term quadratic in LHRS momen-

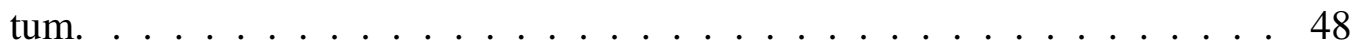

4.11 Coincidence time comparison before (top right) and after calibrations. Note the RF beam structure visible after calibration. . . . . . . . . . . . . . . . . 49

4.12 PMT number vs PMT response before calibration for pion rejection layer 2 in the LHRS. . . . . . . . . . . . . . . . . . . . 50

4.13 PMT number vs calibrated PMT response for pion rejection layer 1 in the LHRS. . . . . . . . . . . . . . . . . . 50

4.14 PMT number vs calibrated PMT response for pion rejection layer 2 in the LHRS. . . . . . . . . . . . . . . . . 51

4.15 PMT number vs calibrated PMT response for the preshower calorimeter in the RHRS. . . . . . . . . . . . . . . . . . . . . 51

4.16 PMT number vs calibrated PMT response for the shower calorimeter in the RHRS.

4.17 Response of the individual Cherenkov PMTs in arbitrary units. Note the wide pedestals in channels half the channels, including the central acceptance. 53

4.18 Expanded view of the Cherenkov pedestal issues. The left column shows problematic PMT pedestals with multipole peaks. The right column pedestals are much narrower and rise more steeply.

5.1 Coincidence time of flight for detected particles at JLab in PMT channels ( 2 channels $=1 \mathrm{~ns})$. The full width at half maximum of the peak is approximately $1.5 \mathrm{~ns}$, and the $2 \mathrm{~ns}$ RF structure of the background is clearly visible. 
5.2 Coincidence time of flight for detected particles at MAMI. The full width at half maximum of the peak is approximately $1.1 \mathrm{~ns} . \ldots \ldots 7$

5.3 The electron arm preshower detector over electron momentum cut spectrum is shown with and without electrons being selected by the Cherenkov detector. . . . . . . . . . . . . . . . . . 58

5.4 The electron arm cherenkov detector spectrum is shown with and without electrons being selected by the preshower detector. . . . . . . . . . . 59

5.5 Lead glass calorimeter particle identification process for electron arm at JLab. The indicated region was cut from the data. . . . . . . . . . . . 59

5.6 Lead glass calorimeter particle identification process for proton arm at JLab. Events below indicated line are cut from the data. . . . . . . . . . 60

5.7 Coincidence time plot for events discarded in the cut shown in figure 5.6. Axes are counts per bin vs channels. Lack of a timing peak indicates events eliminated are not from timing peak. . . . . . . . . . . . 61

5.8 Missing mass peak for VCS events in MAMI experiment A1/03-12 before and after subtracting the pion tail. $\ldots \ldots \ldots \ldots$

5.9 Analysis of spectrometer magnet momentum settings. Each line represents one setting for an asymmetry pair where the spectrometers are moved, but the magnet settings are not changed. For each point, the electron momentum is fixed, and the proton momentum setting tuned to match the missing mass peak at the theoretical value. The intersection of the lines gives the optimized momentum settings. . . . . . . . . . . . . . . . . 62

5.10 Corrections to the missing mass peak from spectrometer momentum optimization and energy loss corrections provide much better agreement in the missing mass spectrum with theory. $\ldots \ldots \ldots \ldots$ 
5.11 Instability in the VCS missing mass peak during the MAMI beamtime. . . . 64

5.12 Optimized electron arm momenta for MAMI beamtime. Note the total vertical scale represents $0.1 \%$ fluctuation from the center. . . . . . . . . . 64

5.13 Optimized proton arm momenta for MAMI beamtime. Note the total vertical scale represents less than $0.1 \%$ fluctuation from the center. . . . . . . . 65

5.14 Comparison of initial and optimized missing mass evolution during the MAMI experiment. . . . . . . . . . . . . . . . 65

$5.15 \mathrm{JLab}$ target length analysis. . . . . . . . . . . . . . . . . 66

5.16 MAMI target density during beam time. Tails at either end correspond to target commissioning and decomissioning. Fluctuations per run are less than $0.25 \%$. . . . . . . . . . . . . . . . . . . . 68

5.17 Filled Red: single track cut cTOF spectrum. Blue: multi track cut cTOF spectrum scaled to match BG plateau height. . . . . . . . . . . . . 71

5.18 Plot comparing the electron particle identification spectrum (energy deposited in the preshower layer divided by electron momentum) for events in the timing peak, and discarded multiple track events. . . . . . . . . . . 72

5.19 Example demonstrating multiplicity contributions from tracks in the VDC and hits in the S2 plane PMT TDC readout. . . . . . . . . . . . 73

5.20 Filled Red: single track cut cTOF spectrum. Blue: Complete cTOF spectrum after multi-track analysis. . . . . . . . . . . . . . . . 74

5.21 Phase space overlap plots for inside and outside kinematics at $\mathrm{Q}^{2}=0.09$ $(\mathrm{GeV} / \mathrm{C})^{2}$. The panels show $\mathrm{Q}^{2}$ vs $\mathrm{W}, \theta_{p q}^{*} \mathrm{vs} \mathrm{W}, \theta_{p q}^{*} \mathrm{vs}^{2}$, and $\theta_{p q}^{*} \mathrm{vs} \phi_{p q}^{*} .76$

5.22 Normalized cross section vs cut fraction . . . . . . . . . . . 78 
5.23 Comparisson of data and simulation for $\mathrm{W}, Q^{2}, \theta_{p q}^{*}$, and $\phi_{p q}^{*}$ yields in spectrometer. . . . . . . . . . . . . . . . 80

5.24 Comparisson of data and simulation for reconstructed mass of undetected pion. ...................... 8

6.1 $\mathrm{Q}^{2}$ evolution of parallel cross section of JLab E08-010 at W $=1232 \mathrm{MeV}$. The Mainz point is from [38]. Errorbars show statistical uncertainty. . . . . 86

6.2 W evolution of parallel cross section of JLab E08-010 at $\mathrm{Q}^{2}=0.13(\mathrm{GeV} / \mathrm{c})^{2}$. Errorbars show statistical uncertainty. . . . . . . . . . . . . . . . . 87

6.3 Summary plots for algebraic extractions at $\mathrm{Q}^{2}=0.040(\mathrm{GeV} / \mathrm{c})^{2}$. Top panel: Asymmetry vs center of mass proton scattering angle $(\theta)$. Middle Panel: longitudinal-transverse partial cross section vs $\theta$. Bottom panel: $\sigma_{0}+\varepsilon \cdot \sigma_{T T}$ vs $\theta$, note $\sigma_{T T} \rightarrow 0$ as $\theta \rightarrow 0$. Inside errorbars show statistical uncertainty, outside show statistical plus systematic uncertainties added in quadrature. ...................... 88

6.4 Summary plots for algebraic extractions at $\mathrm{Q}^{2}=0.090(\mathrm{GeV} / \mathrm{c})^{2}$. Top panel: Asymmetry vs center of mass proton scattering angle $(\theta)$. Middle Panel: longitudinal-transverse partial cross section vs $\theta$. Bottom panel: $\sigma_{0}+\varepsilon \cdot \sigma_{T T}$ vs $\theta$, note $\sigma_{T T} \rightarrow 0$ as $\theta \rightarrow 0$. Inside errorbars show statistical uncertainty, outside show statistical plus systematic uncertainties added in quadrature. . . . . . . . . . . . . . . . . . . 89

6.5 Summary plots for algebraic extractions at $\mathrm{Q}^{2}=0.130(\mathrm{GeV} / \mathrm{c})^{2}$. Top panel: Asymmetry vs center of mass proton scattering angle $(\theta)$. Middle Panel: longitudinal-transverse partial cross section vs $\theta$. Bottom panel: $\sigma_{0}+\varepsilon \cdot \sigma_{T T}$ vs $\theta$, note $\sigma_{T T} \rightarrow 0$ as $\theta \rightarrow 0$. Inside errorbars show statistical uncertainty, outside show statistical plus systematic uncertainties added in quadrature. . . . . . . . . . . . . . . . . . . 9 90 
6.6 $\mathrm{Q}^{2}$ evolution of the $M_{1+}^{3 / 2}$ and $E_{1+}^{3 / 2}$ multipoles. Data from the current work and $[11,22,24,37,39]$ are shown with their statistical and systematic uncertainties added in quadrature. Theoretical predictions from MAID [32, 33], DMT [31], SAID [35], Sato-Lee [30], and the large-Nc calculation [77] are also shown. . . . . . . . . . . . . . . . 93

6.7 $\mathrm{Q}^{2}$ evolution of the CMR $\left(S_{1+}^{3 / 2} / M_{1+}^{3 / 2}\right)$. Data from the current work, and $[13,22,24,36,37,39]$ are shown with their statistical and systematic uncertainties added in quadrature. The theoretical predictions of MAID [32, 33], DMT [31], SAID [35], Sato-Lee [30], Capstick [8], HQM [49], the LatticeQCD calculation [27], the large-Nc calculation [77], the DSEM [78], the ChEFT of Pascalutsa-Vanderhaegen (PV) [47] and the Gail-Hemmert (GH) [48] are also shown. . . . . . . . . . . . . . . . . 95

6.8 Asymmetries extracted at from VCS cross sections $\mathrm{W}=1232 \mathrm{MeV}$ and $\mathrm{Q}^{2}$ $=0.2(\mathrm{GeV} / \mathrm{c})^{2}$ from MAMI A1/03-12 . . . . . . . . . . . 97

6.9 $\mathrm{Q}^{2}$ evolution of the CMR $\left(S_{1+}^{3 / 2} / M_{1+}^{3 / 2}\right)$. Data from the current work, and $[13,22,24,36,37,39]$ are shown with their statistical and systematic uncertainties added in quadrature. The theoretical predictions of MAID [32, 33], DMT [31], SAID [35], Sato-Lee [30], Capstick [8], HQM [49], the LatticeQCD calculation [27], the large-Nc calculation [77], the DSEM [78], the ChEFT of Pascalutsa-Vanderhaegen (PV) [47] and the Gail-Hemmert (GH) $[48]$ are also shown. . . . . . . . . . . . . . . . . . . . 99

6.10 Generalized electric polarizability extracted at from VCS cross sections at $\mathrm{W}=1232 \mathrm{MeV}$ and $\mathrm{Q}^{2}=0.2(\mathrm{GeV} / \mathrm{c})^{2}$ from MAMI A1/03-12. World data [51-55] shown with open boxes were extracted with LEX, and open circles from DR. The solid curve corresponds to HBChPT [81, 82]. The dashed line shows dipole fall off from the DR calculation. . . . . . . . . . . . 100 


\section{LIST OF TABLES}

2.1 Summary of lowest nucleon excitations from [58]. Shaded rows show transition of interest for these experiments. The subscripts $\gamma, \mathrm{N}, \mathrm{R}$, and $\pi$ refer to quantum numbers of the virtual photon, the nucleon, the excited resonance and the pion, respectively. $\mathrm{L}$ and $\mathrm{l}$ are the photon and pion angular momenta, s labels spins, $\mathrm{I}$ isospin and $\mathrm{J}$ the total angular momentum as described in the text. . . . . . . . . . . . . . . . 12

2.2 Notation for the six dipole GPs. The polarization state notation of the first column is described in the text. The multipole notation in the second column uses the magnetic and longitudinal multipoles. The third column gives the correspondence in the RCS limit, defined by $Q^{2} \rightarrow 0 \ldots \ldots \ldots$

2.3 Kinematic values for $Q^{2}, \mathrm{~W}$, proton polar angle $\theta_{p q}^{*}$, and proton azimuthal

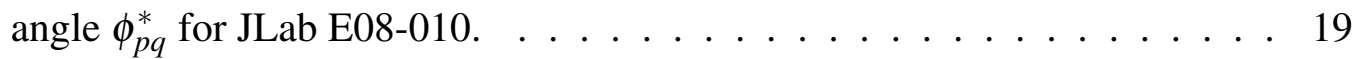

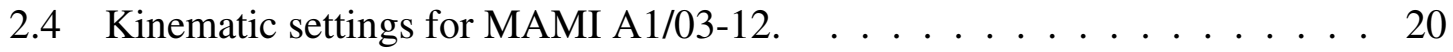

3.1 Summary of targets present during experiment. Thickness shown for the upstream, downstream, and side walls. Data from [67] . . . . . . . 25

3.2 Summary of HRS performance. Data from [65] . . . . . . . . . . 26

3.3 Summary of A1 spectrometer performance. Data from [73] . . . . . . . 35 
3.4 Summary of VDCs in A1 spectrometers. Data from [71] . . . . . . . . 38

5.1 Luminosity factors at JLab. Density Correction is a correction to 0.0723 g/cc. Uncertainties are given as a percentage of the value. . . . . . . . . . 67

5.2 Percentage of events passing each cut. . . . . . . . . . . 70

5.3 Efficiencies and correction factors at JLab. All corrections are presented as a percentage of the cross section. Uncertainties are given as a percentage of the correction. Kinematics with asterisk denote $15 \mathrm{~cm}$ target. All others use $4 \mathrm{~cm}$ target. The density correction shown is the average across all runs. Corrections are made individually for each run according to the average beam current. . . . . . . . . . . . . . . . . . 77

5.4 Cut definitions for the kinematic variables $\mathrm{W}, Q^{2}$, and $\theta_{p q}^{*}$. The cut widths are common among all kinematics sharing the same central value. For $\theta_{p q}^{*}$ there are two widths, with all non-zero central values sharing the same cut. . 79

5.5 Summary of systematic and model uncertainties at JLab. . . . . . . . . 83

5.6 Summary of systematic and model uncertainties at MAMI. . . . . . . . . . 83

6.1 Measured pion electroproduction cross sections from JLab E08-010. Uncertainties are statistical, model, and systematic. . . . . . . . . . 85

6.2 Measured pion electroproduction parallel cross sections from JLab E08010. Uncertainties are statistical, model, and systematic. . . . . . . . . 85

6.3 Pion electroproduction asymmetries and partial cross sections from JLab E08-010 measured at the $\Delta$ resonance $(\mathrm{W}=1232 \mathrm{MeV})$. Uncertainties are statistical, model, and systematic. . . . . . . . . . . . . . . 91 
6.4 Pion electroproduction extracted multipoles from JLab E08-010 measured at the $\Delta$ resonance $(\mathrm{W}=1232 \mathrm{MeV})$. Uncertainties are statistical, systematic, and model. The data at $\mathrm{Q}^{2}=0.04(\mathrm{GeV} / \mathrm{c})^{2}$ show insufficient sensitivity to the EMR for a meaningful fit result to be presented. . . . . . . . . . . . 92

6.5 Measured photon electroproduction cross sections from MAMI A1/03-12. Uncertainties are statistical, and systematic. . . . . . . . . . . . . . 96

6.6 Asymmetries extracted at from VCS cross sections $\mathrm{W}=1232 \mathrm{MeV}$ and $\mathrm{Q}^{2}$ $=0.2(\mathrm{GeV} / \mathrm{c})^{2}$ from MAMI A1/03-12. Uncertainties are statistical, and

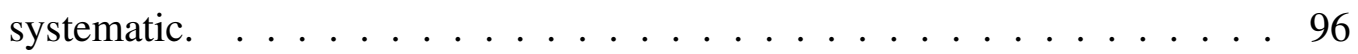

6.7 Results extracted at from VCS cross sections $\mathrm{W}=1232 \mathrm{MeV}$ and $\mathrm{Q}^{2}=$ $0.2(\mathrm{GeV} / \mathrm{c})^{2}$ from MAMI A1/03-12. Uncertainties are statistical, and sys-

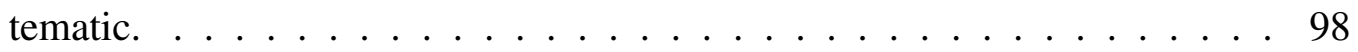




\section{CHAPTER 1}

\section{INTRODUCTION}

Hadrons are the smallest objects known to have finite size and shape, and are the backbone of all the matter seen across the universe. Hadrons are commonly broken into two groups: baryons (systems of three bound quarks) and mesons (bound quark-antiquark pairs), though this system should be expanded to include recently found tetra- and penta- quark exotic states $[1,2]$. Nucleons are the ground state baryons, and the proton is the only baryon that is stable. This makes the proton an important test bed for exploring quark interactions.

As spin 1/2 particles, nucleons are methematically prohibited from having a quadrupole moment. Direct observation of non-spherical behavior is possible through the transition to the $\Delta$ excited state (spin $3 / 2$ ), where the quadrupole moments no longer cancel out. Indeed, pion electroproduction experiments [3-50] have demonstrated the presence of $E_{1+}^{3 / 2}$ and $S_{1+}^{3 / 2}$ multipoles (or E2 and C2 photon absorption quadrupoles, respectively) in the predominantly magnetic multipole $M_{1+}^{3 / 2}$ (or M1 dipole) $\gamma^{*} N \rightarrow \Delta$ transition. Their relative strength is normally quoted in terms of the ratios $\mathrm{EMR}=\operatorname{Re}\left(E_{1+}^{3 / 2} / M_{1+}^{3 / 2}\right)$ and $\mathrm{CMR}$ $=\operatorname{Re}\left(S_{1+}^{3 / 2} / M_{1+}^{3 / 2}\right)$. Previous experimental results are in reasonable agreement with models $[30,31]$ suggesting the presence of non-spherical components in the nucleon wave function.

These amplitudes have been explored up to four momentum transfer squared $Q^{2}=$ $7(\mathrm{GeV} / \mathrm{c})^{2}$ through pion electroproduction H(e,e'p) $\pi^{0}[9-25,36-42]$. Dynamics of the Sato Lee [30] model, however, suggest the pion cloud contribution to the multipoles will be maximized in the low momentum transfer regime, as seen in Figure 1.1.

Focusing at the low momentum transfer regime in Figure 1.2 shows quantum chromodynamic (QCD) models of the quark core (HQM [49] and Capstick [8]) fail to predict the magnitude of multipoles. Additionally, most of the other models predict an increase 


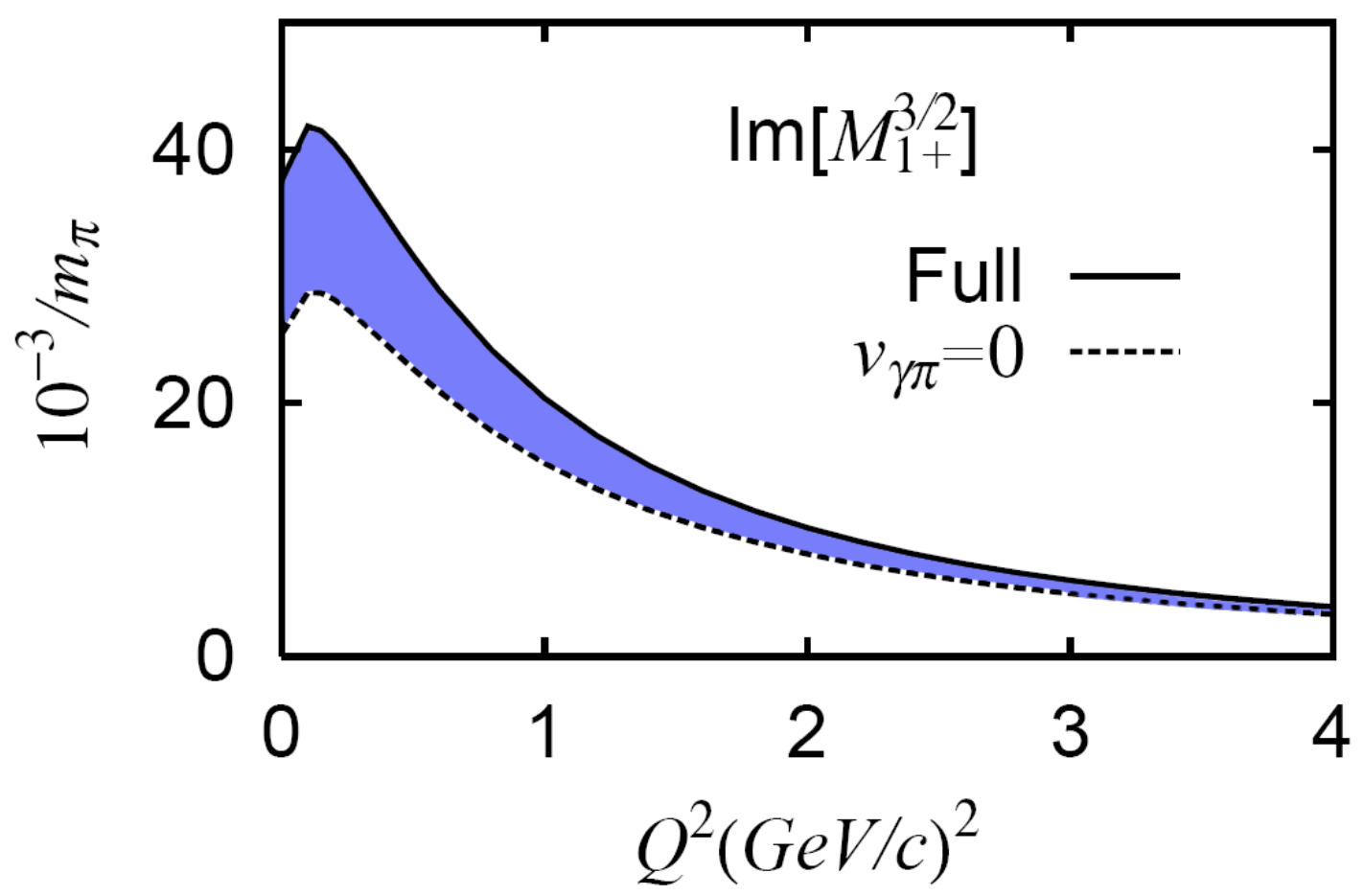

Figure 1.1: Figure from [30] demonstrating enhancement of pion cloud dynamics (shaded region) at low momentum transfer. 
in $\mathrm{CMR}$ as $\mathrm{Q}^{2} \rightarrow 0$ that is not shown in the data. In the case of the Sato Lee model, this increase in CMR corresponds to a situation where the CMR and EMR are equal at the real photon point.

The E08-010 experiment (known as "N-Delta") at Jefferson Lab is designed to map the low momentum transfer regime from $\mathrm{Q}^{2}=0.04$ to $0.13(\mathrm{GeV} / \mathrm{c})^{2}$ with high precision multipole extractions at three points.
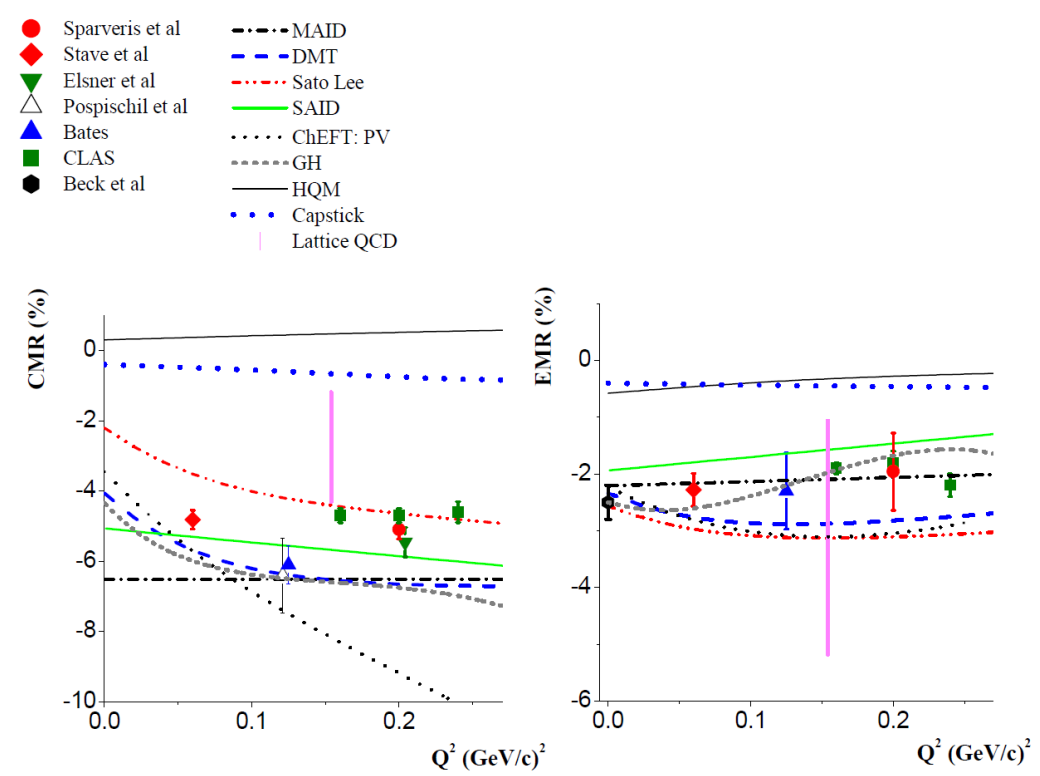

Figure 1.2: World data and predictions for the multipole ratios in the low momentum transfer regime. Data from [13, 22, 24, 36, 37, 39] are shown with their statistical and systematic uncertainties added in quadrature. Theoretical predictions from MAID [32, 33], DMT [31], SAID [35], Sato-Lee [30], Capstick [8], HQM [49], the Lattice-QCD calculation [27], the ChEFT of Pascalutsa-Vanderhaegen (PV) [47] and the Gail-Hemmert (GH) [48].

The nucleon to $\Delta(1232)$ transition can be accessed through three channels. World data in the CMR has been collection through pion electroproduction $\mathrm{H}\left(\mathrm{e}, \mathrm{e}\right.$ 'p) $\pi^{0}$, which has a branching ration of $66 \%$. Similar measurement can be made through the virtual Compton scattering (VCS) reaction channel $\mathrm{H}\left(\mathrm{e}, \mathrm{e}^{\prime} \mathrm{p}\right) \gamma$, but the branching ratio is $<1 \%$ (for completeness, the other $33 \%$ are from $\left.\mathrm{H}\left(\mathrm{e}, \mathrm{e}^{\prime} \pi^{+}\right) \mathrm{n}\right)$. VCS measurements require a different theoretical framework to include different background terms, and the dynamics target proton interacting with the outbound photon. These differences make the measurement of the CMR through VCS a powerful crosscheck of the abilities of the models to isolate the signal of interest from the noise of other processes present in the data. 
The response of the proton to electromagnetic fields is characterized by its electric and magnetic polarizabilities, $\alpha_{E}$ and $\beta_{M}$, respectively. In the pion cloud model of the proton these effects can be naively viewed as is Figure 1.3, where the deformations are a result of simple interactions between the applied fields and the pion cloud or quark core. The electric field applied directly polarizes the pion cloud, resulting in a relatively strong signal. The magnetic field produces opposing effects: paramagnetic alignment proton spin in the quark core, and diamagnetic effect of the pion cloud producing a field opposing the applied field.

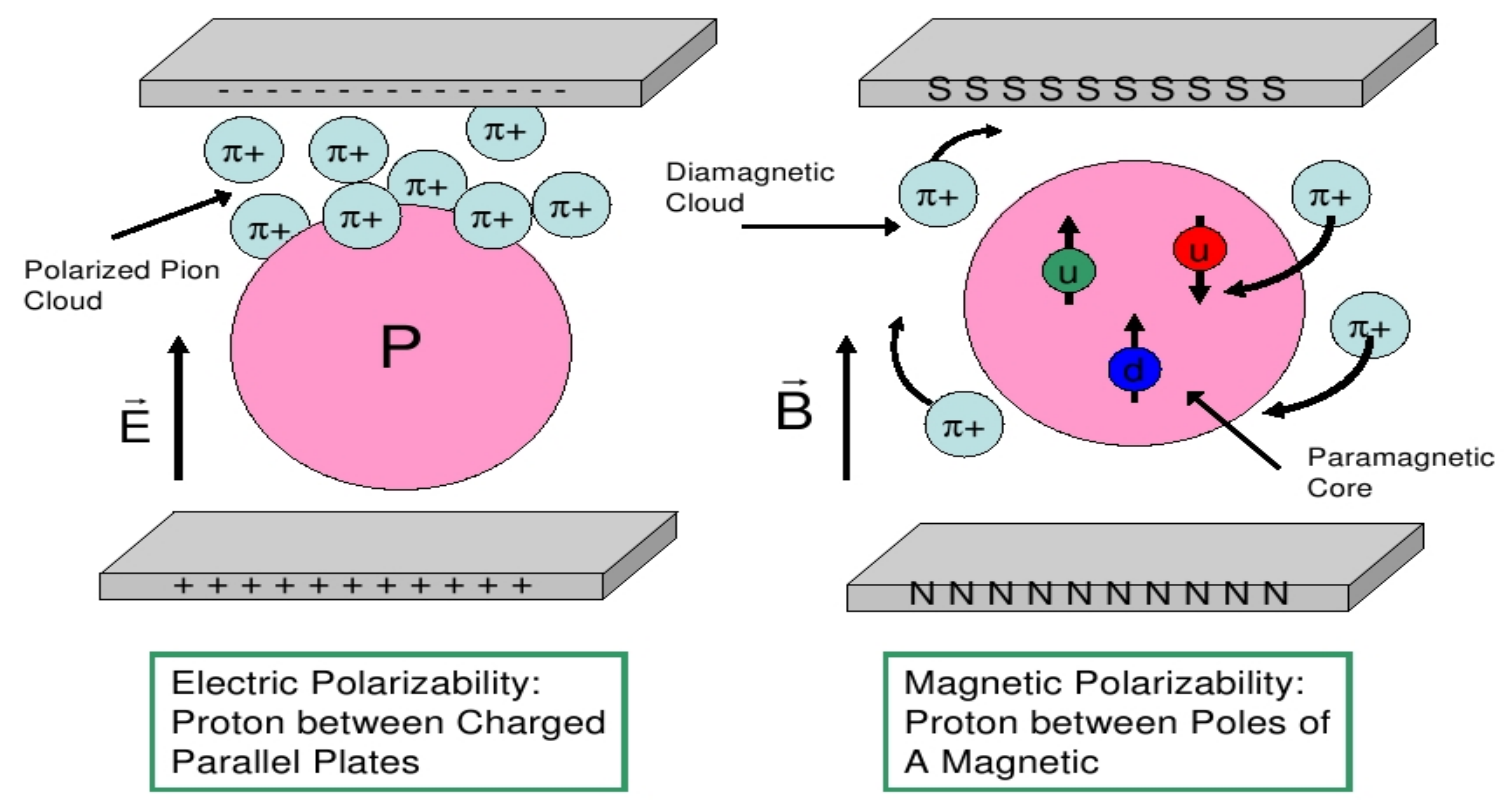

Figure 1.3: Simple model of proton describing its electric and magnetic polarizabilities.

VCS allows the polarizabilities to be generalized as a function of the momentum transfered from the virtual photon mediating the interaction between the scattering electron and proton. Previous experiments [51-55] have demonstrated the magnitude of deformations in the proton are very small (1/1000th the size of the nucleon). Figure 1.4 shows the model predictions in context with the world data. There is a clear discrepancy between predictions and data near $\mathrm{Q}^{2}=0.33(\mathrm{GeV} / \mathrm{c})^{2}$. New advances in Dispersion Relation (DR) [60, 61] calculations allow experiments to be run with higher center of mass energies, where the desired signal is stronger.

Experiment A1/03-12 at the Mainz Microtron (MAMI)is part of an effort to map out the electric generalized polarizability (GP) in the region of the observed discrepancies between data and theory. A1/03-12 will measure both the electric GP and the CMR through the VCS 


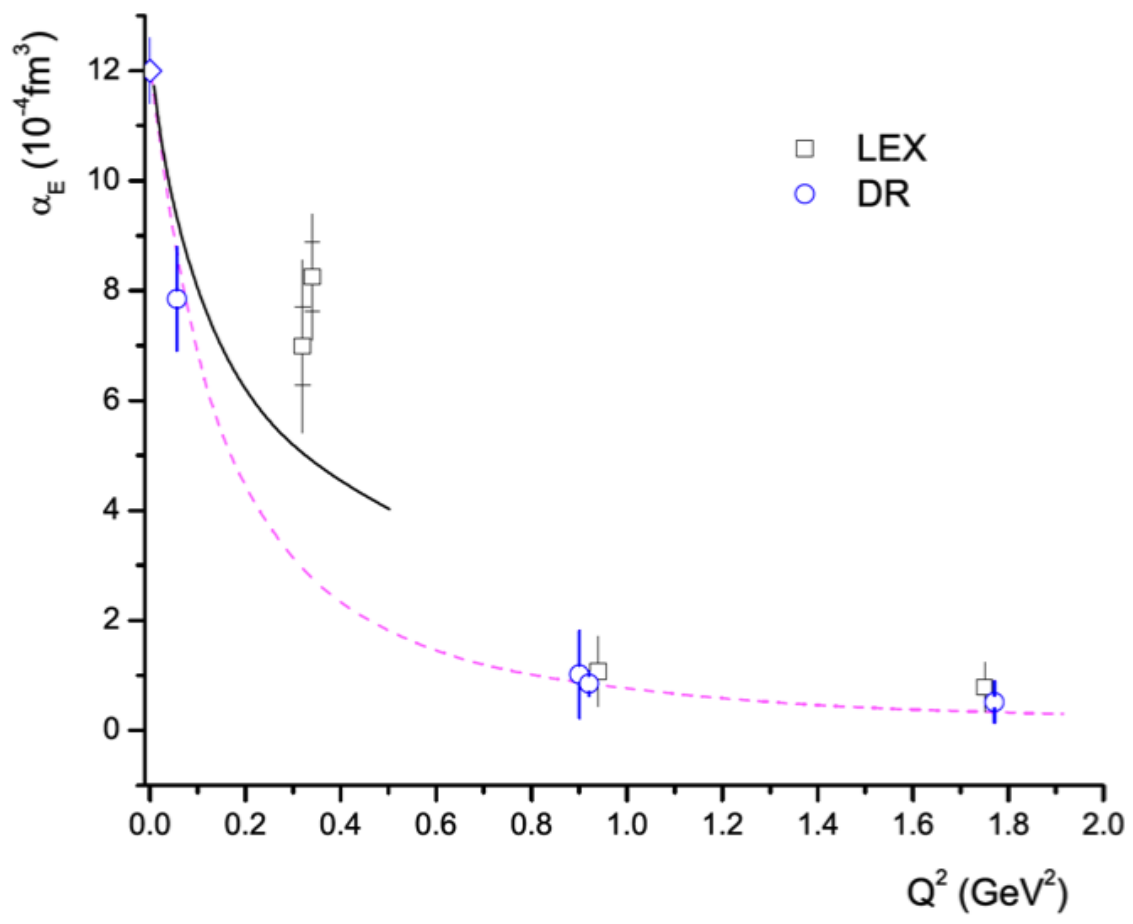

Figure 1.4: World data [51-55] shown with open boxes were extracted with LEX, and open circles from DR. The solid curve corresponds to HBChPT [81, 82]. The dashed line shows dipole fall off from the DR $[60,61]$ calculation. 
channel at $\mathrm{Q}^{2}=0.20(\mathrm{GeV} / \mathrm{c})^{2}$. Previous high precision measurement of the CMR at this point through pion electroproduction will provide a clear indication of how well model extractions of the CMR are understood in this regime. 


\section{CHAPTER 2}

\section{THEORY}

\subsection{Kinematics}

Pion electroproduction and virtual Compton scattering (VCS) have closely related reaction diagrams and kinematical descriptions. Six four-vectors label the particles individually during three stages the reactions are commonly broken into. First in the reaction plane, we have the incident electron, $k_{i}=\left(E_{i}, \vec{k}_{i}\right)$, the scattered electron, $k^{\prime}=\left(E^{\prime}, \overrightarrow{k^{\prime}}\right)$, and the virtual photon, $q=(\omega, \vec{q})$. The reaciton plane contains the target nucleus, $p_{T}=\left(E_{T}, \overrightarrow{p_{T}}\right)$, the scattered proton, $p_{f}=\left(E_{f}, \vec{p}_{f}\right)$, and the undetected pion or photon, $p_{\pi}=\left(E_{\pi}, \vec{p}_{\pi}\right)$ or $p_{\gamma}=$ $\left(E_{\gamma}, \vec{p}_{\gamma}\right)$ in the case of pion electroproduction or VCS, respectively. The electron and proton four vectors are all known through the experiment, while $q$ and $p_{\pi}$ or $p_{\gamma}$ are calculated. $q=k^{\prime}-k_{i}$ defines the momentum transfer direction which is the line of intersection between the scattering and reaction planes. $p_{\pi}$ or $p_{\gamma}=q+p_{T}-p_{f}$ determines which channel of the $\Delta$ decay is being measured in the experiment. Often both channels show up in the data stream of the experiment, at about 100:1 ratio in favor of the pion. Figures 2.1 and 2.2 show how the variables above are related.

Kinematic quantities of interest are derived from these four vectors. The momentum transfer is often summararized by the the frame independent $\mathrm{Q}^{2}=-q^{2}$. The calculation for this is simplified in most experiments since the inbound and outbound electrons are traveling close to the speed of light. Hence, $E_{i} \approx\left|\vec{k}_{i}\right|$ and $E^{\prime} \approx\left|\vec{k}^{\prime}\right|$, providing:

$$
Q^{2}=4 E_{i} E^{\prime} \sin ^{2} \frac{\theta_{e}}{2}
$$




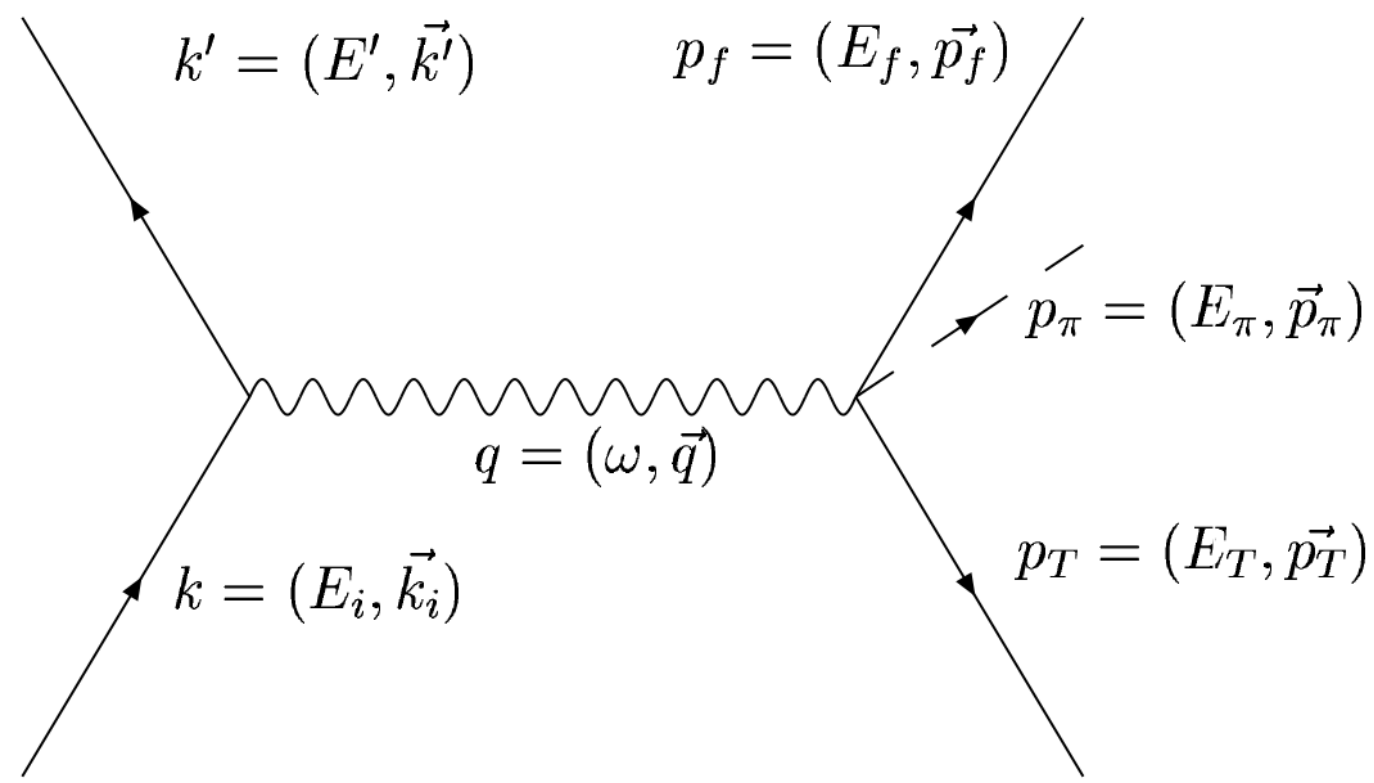

Figure 2.1: Kinematics for pion electroproduction. Figure from [56].

where $\theta_{e}$ is the angle of the scattered electron from the inbound electron in the laboratory frame.

The center of mass energy, $\mathrm{W}$, is also commonly used to describe the system. This can also be quickly calculated from:

$$
W^{2}=\left(q+p_{T}\right)^{2}=-Q^{2}+m_{p}^{2}+2 \omega m_{p}
$$

calculated in the lab frame where the target nucleus is at rest and $p_{T}=\left(m_{p}, \overrightarrow{0}\right)$ with $m_{p}$ being the proton mass.

Properties of the virtual photon are also required. The transverse polarization of the virtual photon, $\varepsilon$, is given by:

$$
\varepsilon=\left(1+\frac{q^{2}}{Q^{2}} \tan ^{2} \frac{\theta_{e}}{2}\right)^{-1}
$$

The virtual photon flux, $\Gamma$ is:

$$
\Gamma=\frac{\alpha}{2 \pi^{2}} \frac{E^{\prime}}{E_{i}} \frac{k_{\gamma}}{Q^{2}} \frac{1}{1-\varepsilon}
$$


where $k_{\gamma}$ is the lab energy required for a real photon to excite the reaction, or the 'photon equivalent energy':

$$
k_{\gamma}=\frac{W^{2}-m_{p}^{2}}{2 m_{p}} .
$$

Several important angles are shown in Figure 2.2. As mentioned earlier, $\theta_{e}$ is the lab frame scattering angle of the electron. $\phi$ is the angle between the scattering and reaction planes. These experiments focus solely on coplanar situations where $\phi=0$ or $\phi=180$ degrees. Finally, $\theta_{\gamma \gamma}$ (or $\theta_{\pi q}$ ) is the scattering angle of the outbound photon (or pion) from the momentum transfer direction $(\vec{q})$. This is often worked in the center of mass frame, and converted to the proton angle $\theta_{p q^{*}}=\pi-\theta_{\pi q^{*}}$ and $\phi_{p q^{*}}=\pi+\theta_{\pi q^{*}}$, or equivalently $\theta_{p q} *=\pi-\theta_{\gamma \gamma} *$ and $\phi_{p q} *=\pi+\theta_{\gamma \gamma} *$.

Finally, it can be useful to discuss results in different reference frames. The Jacobian, J, transforms from the pion center of mass frame to the proton lab frame, where experiments are performed.

$$
J=\frac{d \Omega_{\pi}^{c m}}{d \Omega_{p}^{l a b}}=\frac{p_{f}^{2} \cdot W}{\left|p_{f} *\right| \gamma W\left|p_{f}\right|-E_{f}|q| \mid \cos \theta_{p q}}
$$




\section{REACTION PLANE}

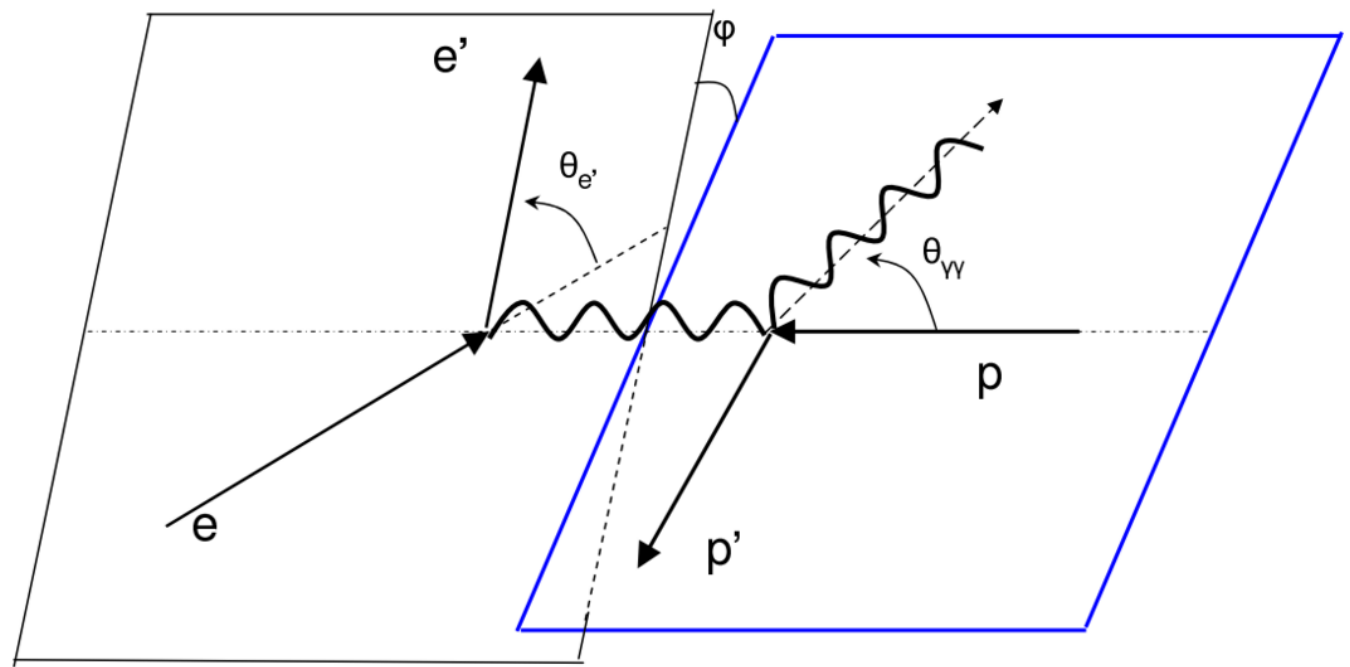

\section{SCATTERING PLANE}

Figure 2.2: Kinematics for VCS. The scattering plane is defined by $\vec{k}_{i} \times \vec{k}^{\prime}$. The reaction plane is defined by $\vec{q} \times \vec{p}_{\gamma}$. Figure from [57].

\subsection{Cross Sections}

The cross section of the $p\left(\vec{e}, e^{\prime} p\right) \pi^{\circ}$ reaction is sensitive to five independent partial responses $\left(\sigma_{T}, \sigma_{L}, \sigma_{L T}, \sigma_{T T}\right.$ and $\left.\sigma_{L T^{\prime}}\right)[34]$ and can be written as:

$$
\begin{aligned}
\frac{d^{5} \sigma}{d \omega d \Omega_{e} d \Omega_{p q}^{c m}}= & \Gamma\left(\sigma_{T}+\varepsilon \cdot \sigma_{L}-v_{L T} \cdot \sigma_{L T} \cdot \cos \phi_{p q}^{*}\right. \\
& \left.+\varepsilon \cdot \sigma_{T T} \cdot \cos 2 \phi_{p q}^{*}-h \cdot v_{L T^{\prime}} \cdot \sigma_{L T^{\prime}} \cdot \sin \phi_{p q}^{*}\right)
\end{aligned}
$$

where $v_{L T}=\sqrt{2 \varepsilon(1+\varepsilon)}$ and $v_{L T^{\prime}}=\sqrt{2 \varepsilon(1-\varepsilon)}$ are kinematic factors, $\varepsilon$ is the transverse polarization of the virtual photon, $\Gamma$ is the virtual photon flux, $h= \pm 1$ is the electron longitudinal polarization, or helicity. The differential cross sections ( $\sigma_{T}, \sigma_{L}, \sigma_{L T}, \sigma_{T T}$, and $\sigma_{L T^{\prime}}$ ) are all functions of the center-of-mass energy $\mathrm{W}$, the four momentum transfer squared $Q^{2}=-q^{2}$ ( $q$ is the momentum transfer four-vector), and the proton center of mass polar angle $\theta_{p q}^{*}$ (measured from the momentum transfer direction) [34]. For convenience, $\sigma_{T}$ and $\sigma_{L}$ are also combined into $\sigma_{0}=\sigma_{T}+\varepsilon \cdot \sigma_{L}$ since they cannot be separated without varying $\varepsilon$.

This formalism can be decomposed further to separate contributions from individual 
components of the wavefunction. This analysis is shown below, and will be useful in extracting the final results.

\subsection{Multipoles}

The excitation process of the scattering experiment can be broken down into distinct logical operations. The virtual photon interacts with the proton, exciting it to a $\Delta^{+}$, which then decays into a proton and either a $\pi^{0}$ or a real photon. The excitation process can be described with a series of vector spherical harmonics as in Equation 2.8 as in [34]. Here, $\lambda$ describes the polarization of the virtual photon, which has spin 1 , relative orbital momentum $\tilde{l}$ with respect to the proton, and $L$ total angular momentum in the initial state. The electric and magnetic transitions correspond to transverse polarizations $\lambda= \pm 1$, while the Coulomb transition corresponds to longitudinal polarization $\lambda=0$. Multipole notation focusing on the virtual photon uses $E L, M L$, and $C L$ for the electric, magnetic, and Coulombic multipoles with angular momentum $L$. The total angular momentum of the initial system is $J=\mid L \pm 1 / 2$.

$$
\mathbf{Y}_{\tilde{l} L M}=\sum_{v} C(1 \lambda, \tilde{l} v \mid L M) \hat{\mathbf{e}}_{\lambda} Y_{\tilde{l} v}(\hat{\mathbf{r}})
$$

Alternatively, one can focus on the final $\pi \mathrm{N}$ system, where the electric, magnetic, and longitudinal multipoles for a given orbital angular momentum, $l$, of the pion with respect to the recoiling proton are $E_{l \pm}, M_{l \pm}$ and $L_{l \pm} . L_{l \pm}$ can also be written as the scalar multipole $S_{l \pm}=\frac{|\overrightarrow{q *}|}{\omega *} L_{l \pm}$, where $\overrightarrow{q *}$ is the momentum of the virtual photon in the center of mass frame, and $\omega *$ is the energy of the virtual photon in the center of mass frame. The \pm refers to whether or not the proton spin and pion orbital momentum are aligned, i.e. total angular momentum of the final system is $J=\mid l \pm 1 / 2$.

Conservation of angular momentum leads to $|L-l| \leq 1$, and parity considerations further refine this for the $\gamma * \mathrm{~N}$ multipoles: $L=l$ for $M L$, and $|L-l|=1$ for $E L$ and $C L$ [34]. Table 2.1 summarizes the lowest nucleon excitations and their multipole descriptions. Note that the $\Delta$ resonance can be reached through multiple multipole transitions. The other transitions will also be present in an experimental data stream, and must be accounted for during the analysis. 
Table 2.1: Summary of lowest nucleon excitations from [58]. Shaded rows show transition of interest for these experiments. The subscripts $\gamma, \mathrm{N}, \mathrm{R}$, and $\pi$ refer to quantum numbers of the virtual photon, the nucleon, the excited resonance and the pion, respectively. $L$ and 1 are the photon and pion angular momenta, s labels spins, I isospin and $\mathrm{J}$ the total angular momentum as described in the text.

\begin{tabular}{|c|c|c|c|c|c|c|c|c|}
\hline$\gamma * \mathrm{~N}$ Multipoles & \multicolumn{2}{|l|}{ Initial State } & \multicolumn{2}{|c|}{ Excited State } & \multicolumn{2}{|c|}{ Final State } & $\pi \mathrm{N}$ Multipoles \\
\hline $\mathrm{C}, \mathrm{E}, \mathrm{M}$ & $\mathrm{L}_{\gamma}^{\pi}$ & $\mathrm{s}_{N}^{\pi}$ & $\mathrm{J}_{R}^{\pi}$ & \multicolumn{2}{|c|}{$\mathrm{N}^{*} \mathrm{I}_{2 I, 2 J} \Delta$} & $\mathrm{s}_{N}^{\pi}$ & $\mathrm{I}_{\pi}^{\pi}$ & $\mathrm{L}_{\ell \pm}, \mathrm{E}_{\ell \pm}, \mathrm{M}_{\ell \pm}$ \\
\hline $\mathrm{C} 0$ & $0^{+}$ & $1 / 2^{+}$ & $1 / 2^{+}$ & $\mathrm{P}_{11}$ & $\mathrm{P}_{31}$ & $1 / 2^{+}$ & $1^{+}$ & $\mathrm{L}_{1-}$ \\
\hline \multirow{2}{*}{$\mathrm{C} 1, \mathrm{E} 1$} & $1^{-}$ & $1 / 2^{+}$ & $1 / 2^{-}$ & $\mathrm{S}_{11}$ & $\mathrm{~S}_{31}$ & $1 / 2^{+}$ & $0^{-}$ & $\mathrm{L}_{0+}, \mathrm{E}_{0+}$ \\
& & $1 / 2^{+}$ & $3 / 2^{-}$ & $\mathrm{D}_{13}$ & $\mathrm{D}_{33}$ & $1 / 2^{+}$ & $2^{-}$ & $\mathrm{L}_{2-}, \mathrm{E}_{2-}$ \\
\hline & $1^{+}$ & $1 / 2^{+}$ & $1 / 2^{+}$ & $\mathrm{P}_{11}$ & $\mathrm{P}_{31}$ & $1 / 2^{+}$ & $1^{+}$ & $\mathrm{M}_{1-}$ \\
\hline $\mathrm{M} 1$ & & $1 / 2^{+}$ & $3 / 2^{+}$ & $\mathrm{P}_{13}$ & $\mathrm{P}_{33}$ & $1 / 2^{+}$ & $1^{+}$ & $\mathrm{M}_{1+}$ \\
\hline $\mathrm{C} 2, \mathrm{E} 2$ & $2^{+}$ & $1 / 2^{+}$ & $3 / 2^{+}$ & $\mathrm{P}_{13}$ & $\mathrm{P}_{33}$ & $1 / 2^{+}$ & $1^{+}$ & $\mathrm{L}_{1+}, \mathrm{E}_{1+}$ \\
& & $1 / 2^{+}$ & $5 / 2^{+}$ & $\mathrm{F}_{15}$ & $\mathrm{~F}_{35}$ & $1 / 2^{+}$ & $3^{+}$ & $\mathrm{L}_{3-}, \mathrm{E}_{3-}$ \\
\hline \multirow{2}{*}{$\mathrm{M} 2$} & $2^{-}$ & $1 / 2^{+}$ & $3 / 2^{-}$ & $\mathrm{D}_{13}$ & $\mathrm{D}_{33}$ & $1 / 2^{+}$ & $2^{-}$ & $\mathrm{M}_{2-}$ \\
& & $1 / 2^{+}$ & $5 / 2^{-}$ & $\mathrm{D}_{15}$ & $\mathrm{D}_{35}$ & $1 / 2^{+}$ & $2^{-}$ & $\mathrm{M}_{2+}$ \\
\hline
\end{tabular}

This experiment focuses on the $\Delta(1232)$ or $P_{33}$ resonance, which is accessed through three multipoles. These multipoles, in turn, have three equivalent notations as in Equation 2.10. The first two notations are as discussed above, while the third is an expansion of the second with the total angular momentum added as a superscript, e.g. $M_{l \pm}^{J}$.

$$
\begin{aligned}
M 1 & \Leftrightarrow M_{1+} \Leftrightarrow M_{1+}^{3 / 2} \\
E 2 & \Leftrightarrow E_{1+} \Leftrightarrow E_{1+}^{3 / 2} \\
C 2 & \Leftrightarrow S_{1+} \Leftrightarrow S_{1+}^{3 / 2}
\end{aligned}
$$

These multipoles of interest vary widely in scale, in particular the M1 multipole is much larger than the others, and is often found interfering in terms that would otherwise be dominated by $\mathrm{E} 2$ or $\mathrm{C} 2$. The electric to magnetic ratio (EMR) and Coulombic to magnetic ratio (CMR) are defined to reduce the $\mathrm{M} 1$ dominance in reported results.

$$
\begin{aligned}
& E M R \equiv \frac{E_{1+}^{3 / 2}}{M_{1+}^{3 / 2}}=\frac{E 2}{M 1} \\
& C M R \equiv \frac{S_{1+}^{3 / 2}}{M_{1+}^{3 / 2}}=\frac{C 2}{M 1}
\end{aligned}
$$


The multipoles are combined with Legendre Polynomials and their derivatives $\left(P_{l}, P_{l}^{\prime}\right.$, and $P_{l}^{\prime \prime}$ ) to make six structure functions from Chew, Goldberger, Low, and Nambu (or CGLNFs)[34].

$$
\begin{aligned}
& F_{1}=\sum_{l \geq 0}\left\{\left(l M_{l+}+E_{l+}\right) P_{l+1}^{\prime}+\left[(l+1) M_{l-}+E_{l-}\right] P_{l-1}^{\prime}\right\} \\
& F_{2}=\sum_{l \geq 1}\left[(l+1) M_{l+}+l M_{l-}\right] P_{l}^{\prime} \\
& F_{3}=\sum_{l \geq 1}\left[\left(E_{l+}-M_{l+}\right) P_{l+1}^{\prime \prime}+\left(E_{l-}+M_{l-}\right) P_{l-1}^{\prime \prime}\right] \\
& F_{4}=\sum_{l \geq 2}\left(M_{l+}-E_{l+}-M_{l-}-E_{l-}\right) P_{l}^{\prime \prime} \\
& F_{5}=\sum_{l \geq 0}\left[(l+1) L_{l+} P_{l+1}^{\prime}-l L_{l-} P_{l-1}^{\prime}\right] \\
& F_{6}=\sum_{l \geq 1}\left[l L_{l-}-(l+1) L_{l+}\right] P_{l}^{\prime}
\end{aligned}
$$

The CGLNFs are then used to build up the response functions[34], labeled according to the polarization components of the virtual photon that they describe. L for longitudinal, $\mathrm{T}$ for transverse, and LT and TT for the interference response between the two polarizations. The prime label (in LT') indicates dependence on beam helicity.

$$
\begin{aligned}
R_{T}= & \left|F_{1}\right|^{2}+\left|F_{2}\right|^{2}+\frac{1}{2} \sin ^{2} \theta\left(\left|F_{3}\right|^{2}+\left|F_{4}\right|^{2}\right) \\
& -\operatorname{Re}\left\{2 \cos \theta F_{1}^{*} F_{2}-\sin ^{2} \theta\left(F_{1}^{*} F_{4}+F_{2}^{*} F_{3}+\cos \theta F_{3}^{*} F_{4}\right)\right\} \\
R_{L}= & \left|F_{5}\right|^{2}+\left|F_{6}\right|^{2}+2 \cos \theta \operatorname{Re}\left\{F_{5}^{*} F_{6}\right\} \\
R_{L T}= & -\sin \theta \operatorname{Re}\left\{\left(F_{2}^{*}+F_{3}^{*}+\cos \theta F_{4}^{*}\right) F_{5}+\left(F_{1}^{*}+F_{4}^{*}+\cos \theta F_{3}^{*}\right) F_{6}\right\} \\
R_{T T}= & \sin ^{2} \theta\left[\frac{1}{2}\left(\left|F_{3}\right|^{2}+\left|F_{4}\right|^{2}\right)+\operatorname{Re}\left\{F_{1}^{*} F_{4}+F_{2}^{*} F_{3}+\cos \theta F_{3}^{*} F_{4}\right\}\right] \\
R_{L T^{\prime}}= & -\sin \theta \operatorname{Im}\left\{\left(F_{2}^{*}+F_{3}^{*}+\cos \theta F_{4}^{*}\right) F_{5}+\left(F_{1}^{*}+F_{4}^{*}+\cos \theta F_{3}^{*}\right) F_{6}\right\}
\end{aligned}
$$

The response functions can then be assembled into the partial cross sections used in Equation 2.7. 


$$
\begin{aligned}
\sigma_{T} & =\frac{\left|\vec{q}^{*}\right|}{k_{\gamma}} R_{T} \\
\sigma_{L} & =\frac{\left|\vec{q}^{*}\right|}{k_{\gamma}} R_{L}\left(\frac{Q^{2}}{w_{c m}^{2}}\right) \\
\sigma_{L T} & =\frac{\left|\vec{q}^{*}\right|}{k_{\gamma}} R_{L T}\left(\frac{Q}{w_{c m}}\right) \\
\sigma_{T T} & =\frac{\left|\vec{q}^{*}\right|}{k_{\gamma}} R_{T T} \\
\sigma_{L T^{\prime}} & =\frac{\left|\vec{q}^{*}\right|}{k_{\gamma}} R_{L T^{\prime}}\left(\frac{Q}{w_{c m}}\right)
\end{aligned}
$$

\subsection{Polarizability}

Polarizability describes the nucleon response to external electromagnetic fields. These are elementary structure constants, just like size and shape, and can be accessed through Compton scattering. The use of virtual Compton scattering (VCS) allows the polarizabilities to be mapped as a function of the length scale of the probe. This scale is set by the virtuality of the incoming virtual photon, defined be $\mathrm{Q}^{2}$. The outgoing photon determines the magnitude of the perturbing electromagnetic fields. The structure dependence is parametrized by the Generalized Polarizabilities (GPs).

The role of the GPs in the cross section can be demonstrated using the Low Energy Theorem [59], expressing the scattering amplitude $T^{e p \gamma}$ as an expansion in powers of $q_{c m}^{\prime}$. This leads to the lowest order:

$$
\frac{d^{5} \sigma}{d k_{\text {elab }}^{\prime} d \Omega_{\text {elab }}^{\prime} d \Omega_{c m}}=\frac{d^{5} \sigma^{B H+B o r n}}{d k_{\text {elab }}^{\prime} d \Omega_{\text {elab }}^{\prime} d \Omega_{c m}}+q_{c m}^{\prime} \cdot \phi \cdot \Psi_{0}+\mathcal{O}\left(q_{c m}^{\prime 2}\right)
$$

where the Beithe-Heitler and Born contributions to the cross section are from bremsstrahlung radiation of the electron and proton, respectively, and can be calculated fully. $\phi$ is a phase space factor, and $\Psi_{0}$ contains the leading order contributions of the GPs. $\Psi_{0}$ is further separated into structure functions in a fashion similar to as in pion electroproduction:

$$
\Psi_{0}=v_{1} \cdot\left(P_{L L}-\frac{1}{\varepsilon} P_{T T}\right)+v_{2} \cdot P_{L T}
$$


Where the structure functions can further be decomposed and related to the GPs:

$$
\begin{aligned}
& P_{L L}=\frac{4 M}{\alpha_{e m}} \cdot G_{E}^{p}\left(Q^{2}\right) \cdot \alpha_{E}\left(Q^{2}\right) \\
& P_{T T}=\left[P_{T T \text { spin }}\right] \\
& P_{L T}=-\frac{2 M}{\alpha_{e m}} \sqrt{\frac{q_{c m}^{2}}{Q^{2}}} \cdot G_{E}^{p}\left(Q^{2}\right) \cdot \beta_{M}\left(Q^{2}\right)+\left[P_{L T \text { spin }}\right]
\end{aligned}
$$

The scalar GPs $\alpha_{E}\left(Q^{2}\right)$ and $\beta_{M}\left(Q^{2}\right)$ are shown extracted for comparison to the real Compton scattering (RCS) limit.

$$
\begin{aligned}
& \alpha_{E}\left(Q^{2}\right)=-P^{(L 1, L 1) 0}\left(Q^{2}\right) \cdot\left(\frac{e^{2}}{4 \pi} \sqrt{\frac{3}{2}}\right) \\
& \beta_{M}\left(Q^{2}\right)=-P^{(M 1, M 1) 0}\left(Q^{2}\right) \cdot\left(\frac{e^{2}}{4 \pi} \sqrt{\frac{3}{8}}\right)
\end{aligned}
$$

The spin part of the structure functions is also separated for clarity:

$$
\begin{aligned}
P_{T T \text { spin }}= & -3 G_{M}^{p}\left(Q^{2}\right) \frac{q_{c m}^{2}}{\tilde{q}^{0}} \cdot\left(P^{(M 1, M 1) 1}\left(Q^{2}\right)\right. \\
& -\sqrt{2} \tilde{q}^{0} \cdot P^{(L 1, M 2) 1}\left(Q^{2}\right) \\
P_{L T \text { spin }}= & \frac{3}{2} \frac{q_{c m} \sqrt{Q^{2}}}{\tilde{q}^{0}} G_{M}^{p}\left(Q^{2}\right) \cdot P^{(L 1, L 1) 1}\left(Q^{2}\right)
\end{aligned}
$$

In these Equations 2.35 to $2.41, \alpha_{e m}$ is the fine structure constant, $G_{E}^{p}$ and $G_{M}^{p}$ are the electric and magnetic structure functions of the proton, and $\tilde{q}^{0}$ is the CM energy of the virtual photon in the limit the outgoing center of mass photon energy goes to zero $\left(E_{g} a m m a^{*} \rightarrow 0\right)$.

The GP notation $P^{\left(\rho^{\prime} L^{\prime}, \rho L\right) S}$ uses the polarization state $\rho\left(\rho^{\prime}\right)$ of the initial (final) photon, the angular momentum $L\left(L^{\prime}\right)$ of the transition, and the non spin-flip $(S=0)$ or spin-flip $(S=1)$ of the nucleon. The GPs are divided into two groups: scalar $(\mathrm{S}=0)$ and vector $(\mathrm{S}=1)$, 
with the scalar GPs having a direct analog to the RCS case. The notation and extrapolation of the GPs is summarized in Table 2.2.

Table 2.2: Notation for the six dipole GPs. The polarization state notation of the first column is described in the text. The multipole notation in the second column uses the magnetic and longitudinal multipoles. The third column gives the correspondence in the RCS limit, defined by $Q^{2} \rightarrow 0$.

\begin{tabular}{|c|c|c|}
\hline$P^{\left(\rho^{\prime} L^{\prime}, \rho L\right) S}\left(q_{c m}\right)$ & $P^{(f, i) S}\left(q_{c m}\right)$ & RCS limit \\
\hline$P^{(01,01) 0}$ & $P^{(L 1, L 1) 0}$ & $-\frac{4 \pi}{e^{2}} \sqrt{\frac{2}{3}} \alpha_{E}$ \\
\hline$P^{(11,11) 0}$ & $P^{(M 1, M 1) 0}$ & $-\frac{4 \pi}{e^{2}} \sqrt{\frac{8}{3}} \beta_{M}$ \\
\hline$P^{(01,01) 1}$ & $P^{(L 1, L 1) 1}$ & 0 \\
\hline$P^{(11,11) 1}$ & $P^{(M 1, M 1) 1}$ & 0 \\
\hline$P^{(01,12) 1}$ & $P^{(L 1, M 2) 1}$ & $-\frac{4 \pi}{e^{2}} \frac{\sqrt{2}}{3} \gamma_{3}$ \\
\hline$P^{(11,02) 1}$ & $P^{(M 1, L 2) 1}$ & $-\frac{4 \pi}{e^{2}} \frac{2 \sqrt{2}}{3 \sqrt{3}}\left(\gamma_{2}+\gamma_{4}\right)$ \\
\hline
\end{tabular}

\subsection{Models}

\subsubsection{Pion Electroproduction}

Many models describing pion electroproduction off the proton have been developed and are evolving as more data becomes available. The models can largely be classified into two distinct categories: those that focus on the quark-gluon core as described directly via quantum chromodynamics (QCD), and those that also include effects from a pion cloud. Despite these core differences, both types of models predict the multipole amplitudes of the proton, then combine them to form cross section predictions for pion electroproduction and the competing background processes of the experiment.

The model of Capstick [8] and hypercentral quark model (HQM) [49] are two examples of QCD models that only include the quark-gluon core degrees of freedom. These models predict very small magnitudes for the CMR and EMR at low $\mathrm{Q}^{2}$, less than $1 \%$, but world data from CLAS, Bates, and MAMI [cite] clearly show magnitudes larger than $4 \%$.

A mechanism describing this discrepancy is explained by Sato and Lee (SL) using a dynamical model [30]. Their model shows agreement with QCD from "bare quark" contributions, and introduces a pion cloud that accounts for the dynamics shown in the data 
at low momentum transfer. The pion cloud concept is used in three other models used to extract results in this thesis.

MAID and SAID are two computational models based on scattering amplitudes with parameterizations. MAID is derived from the Mainz Unitary Isobar Model [32, 33] and SAID is derived from a phase shift analysis of the world data[35]. The Dubna Mainz Taipei (DMT) model is a dynamical model with the same resonance terms as the MAID model, but different background terms [31].

\subsubsection{Virtual Compton Scattering}

Models of VCS must include information from both multipole amplitudes similar to those of pion electroproduction, and also information about the GPs. The two most commong VCS models are the Low Energy Expansion (LEX) [59] and Dispersion Relations (DR) model $[60,61]$. In LEX, the scattering matrix is rewritten as an expansion of the scattering matrix in powers of the outgoing photon energy. This is model independent, and widely applicable considering sufficient number of terms are included. Including restrictions on the outgoing photon energy (i.e. below pion threshold) and assuming single photon exchange collapses the expansion to first order terms, leading to 2.29), the typical LEX model prediction.

The DR model takes a different expansion of the scattering matrix that is not energy dependent. This set of tensors is chosen with respect to symmetry in photon crossing and are constrained by charge conjugation to provide many cancelling terms to simplify calculations, particularly in the real Compton scattering limit $[60,61]$. At nonzero $\mathrm{Q}^{2}$, the invariant tensors of the DR model are written with dispersive integrals from [60] of the form:

$$
\operatorname{ReF}_{i}^{N B}\left(Q^{2}, v, t\right)=\frac{2}{\pi} \int_{v_{t h r}}^{+\mathrm{inf}} d v^{\prime} \frac{v^{\prime} \operatorname{Im}_{s} F_{i}\left(Q^{2}, v^{\prime}, t\right)}{v^{\prime 2}-v^{2}}
$$

where $\operatorname{ReF}_{i}^{N B}\left(Q^{2}, v, t\right)$ are the Non-Born parts of the invariant amplitudes, $\operatorname{Im}_{S} F_{i}\left(Q^{2}, v^{\prime}, t\right)$ are the discontinuities across s-channel cuts of the VCS process, and $v_{t h r}$ is the pion threshold energy $v_{t h r}=m_{\pi}+\left(m_{\pi}^{2}+t / 2+Q^{2} / 2\right) /(2 M)$, with $\mathrm{m}_{\pi}$ and $\mathrm{M}$ being the pion and proton masses and the typical Mandelstam variable.

The dispersive integral shown in Equation 2.42 is solved using unitarity, but is limited to the $\pi \mathrm{N}$ intermediate states, presumed dominant in VCS up to the $\Delta(1232)$ resonance 
region. The calculation uses pion photo- and electroproduction multipoles [60] in which both resonant and non-resonant production mechanisms are included. However, there are additional physics above the $\pi \mathrm{N}$ states, which requires experimental data to include. Two of these extra integrals depend on the scalar GP $\beta_{M}$ and the sum $\left(\beta_{M}+\alpha_{E}\right)$, which can be separated into the known values of the scalar GP and the $\pi \mathrm{N}$ contribution at the RCS limit, as well as a scaling factor to be tuned in the current experiment.

$$
\begin{aligned}
& \beta_{M}\left(Q^{2}\right)=\beta^{\pi N}\left(Q^{2}\right)+\Delta \beta \\
& \Delta \beta=\frac{\left[\beta^{\exp }-\beta^{\pi N}\right]_{Q^{2}=0}}{\left(1+Q^{2} / \Lambda_{\beta}^{2}\right)^{2}} \\
& \alpha_{E}\left(Q^{2}\right)=\alpha^{\pi N}\left(Q^{2}\right)+\Delta \alpha \\
& \Delta \alpha=\frac{\left[\alpha^{\exp }-\alpha^{\pi N}\right]_{Q^{2}=0}}{\left(1+Q^{2} / \Lambda_{\alpha}^{2}\right)^{2}}
\end{aligned}
$$

The form of the scaling factor is a dipole drop off from the RCS limits. This form is completely arbitrary, and the mass parameters $\Lambda_{\alpha}$ and $\Lambda_{\beta}$ can be tuned separately for every value of $\mathrm{Q}^{2}$, yielding arbitrary functional forms for the scalar GPs as functions of momentum transfer.

\subsection{Measurements}

\subsubsection{Pion Electroproduction}

Sequential measurements varying only $\theta_{p q}^{*}$ and $\phi_{p q}^{*}$ were made at each $Q^{2}$ setting to separate the partial cross sections. For each $\theta_{p q}^{*}$ setting the proton spectrometer was sequentially placed at $\phi_{p q}^{*}=0^{\circ}$ and $180^{\circ}$, while keeping the electron position fixed, and maintaining a constant momentum setting for both spectrometers. Thus, $\sigma_{L T}$ and the $\sigma_{0}+\varepsilon \cdot \sigma_{T T}$ responses are separated according to: 


$$
\begin{array}{r}
\sigma_{L T}=\frac{\sigma_{\phi_{p q}=0}-\sigma_{\phi_{p q}=\pi}}{2 \sqrt{2 \varepsilon(1+\varepsilon)}} \\
\sigma_{0}+\varepsilon \cdot \sigma_{T T}=\frac{\sigma_{\phi_{p q}=0}+\sigma_{\phi_{p q}=\pi}}{2}
\end{array}
$$

The in-plane azimuthal asymmetry of the cross section with respect to the momentum transfer direction,

$$
A_{\left(\phi_{p q}=0, \pi\right)}=\frac{\sigma_{\phi_{p q}=0}-\sigma_{\phi_{p q}=\pi}}{\sigma_{\phi_{p q}=0}+\sigma_{\phi_{p q}=\pi}}
$$

was also determined. For the pair of $\phi_{p q}^{*}=0^{\circ}$ and $180^{\circ}$ measurements the cross sections, responses, and asymmetries were obtained with the phase space matched in $\left(\mathrm{W}, Q^{2}, \theta_{p q}^{*}\right)$. Measurements of the parallel cross section $\sigma_{\text {thet }_{p q}=0}$ were also performed in the range of $\mathrm{W}=1170 \mathrm{MeV}$ to $1232 \mathrm{MeV}$. The complete list of kinematic settings can be seen in Table 2.3.

Table 2.3: Kinematic values for $Q^{2}, \mathrm{~W}$, proton polar angle $\theta_{p q}^{*}$, and proton azimuthal angle $\phi_{p q}^{*}$ for JLab E08-010.

\begin{tabular}{|c|c|c|c|}
\hline$Q^{2}(\mathrm{GeV} / \mathrm{c})^{2}$ & $\mathrm{~W}(\mathrm{MeV})$ & $\theta_{p q}^{*}\left(^{\circ}\right)$ & $\phi_{p q}^{*}\left(^{\circ}\right)$ \\
\hline 0.04 & 1232 & 0 & - \\
\hline 0.04 & 1232 & 35 & 0 \\
\hline 0.04 & 1232 & 35 & 180 \\
\hline 0.09 & 1232 & 0 & - \\
\hline 0.09 & 1232 & 47 & 0 \\
\hline 0.09 & 1232 & 47 & 180 \\
\hline 0.13 & 1232 & 0 & - \\
\hline 0.13 & 1232 & 32 & 0 \\
\hline 0.13 & 1232 & 32 & 180 \\
\hline 0.13 & 1232 & 52 & 0 \\
\hline 0.13 & 1232 & 52 & 180 \\
\hline 0.13 & 1170 & 0 & - \\
\hline 0.13 & 1200 & 0 & - \\
\hline
\end{tabular}




\subsubsection{Virtual Compton Scattering}

In plane asymmetries are the focus of the VCS measurements at MAMI A1/03-12. Four kinematics, split into two pairs as shown in Table 2.4 were made. These measurements are at the $\Delta$ resonance for increased sensitivity to the polarizabilities, and at $\mathrm{Q}^{2}=0.2(\mathrm{GeV} / \mathrm{c})^{2}$, a value not previously explored in electric GP measurements.

Table 2.4: Kinematic settings for MAMI A1/03-12.

\begin{tabular}{|c|c|c|c|}
\hline$Q^{2}(\mathrm{GeV} / \mathrm{c})^{2}$ & $\mathrm{~W}(\mathrm{MeV})$ & $\theta_{\gamma \gamma}^{*}\left(^{\circ}\right)$ & $\phi_{\gamma \gamma}^{*}\left({ }^{\circ}\right)$ \\
\hline 0.20 & 1232 & 128 & 0 \\
\hline 0.20 & 1232 & 128 & 180 \\
\hline 0.20 & 1232 & 138 & 0 \\
\hline 0.20 & 1232 & 138 & 180 \\
\hline
\end{tabular}

\subsection{Result Extraction}

Two methods are used to extract the multipole amplitudes and the polarizabilities from the experimental cross sections. The first is A Model Independent Analysis Scheme (AMAIS) developed by Papa Stiliaris and Papanicolas [62]. This is a Monte Carlo fitting method where the fit parameters are all varied independently from one another. In a first pass of the data, the pion electroproduction multipoles up to $\mathrm{L}=5$ were all included to check that sensitivity to the data was limited to the three multipoles of choice ( $E_{l \pm}, M_{l \pm}$ and $L_{l \pm}$ ). Once this check is completed, a fit on just those three parameters was performed with the real and imaginary parts varying freely, while keeping the others fixed at the MAID predicted values (only one model is used, having just shown the result is not sensitive to this choice). These results were used to verify no local minima or other was influencing the second fitting algorithm described next.

The final fitting was performed using Minuit[63] in the method described in [56]. For the JLab data, the $E_{l \pm}, M_{l \pm}$ and $L_{l \pm}$ multipoles are varied as a fraction of the DMT, MAID, SAID, and Sato Lee model predictions, while the rest are fixed at the predicted value. This gives a fit result that is dependent on the model used, allowing an analysis of the model uncertainties in the same manner as previous studies [19, 37, 38, 42, 56]. 


\section{CHAPTER 3}

\section{EQUIPMENT}

\subsection{Jefferson Lab}

The experiment ran in February 27th to March 7th, 2011 at Hall A of Thomas Jefferson National Accelerator Facility (Jefferson Lab) in Newport News Virginia before the $12 \mathrm{GeV}$ upgrade. E08-010, or "N-Delta" ran as part of a series of four similar experiments that shared beamtime, detectors, and shift personnel.

\subsubsection{The Electron Beam}

The electron beam at Jefferson Laboratory (JLab) is known provided by the Continuous Electron Beam Accelerator Facility (CEBAF), consisting of a racetrack design beginning at the injector site, passing linear accelerators in the two straights, recirculation arcs in the curves, and finishing at four experimental halls, see Figure 3.1. The CEBAF accelerates packets of electrons through as many as five laps, and can deliver up to a $5.7 \mathrm{GeV}$ beam at currents up to $150 \mu \mathrm{A}$ [64]. The polarization vector of the electrons in the beam is controlled with a Wein filter at the injection site. For this experiment a 10-80 $\mu \mathrm{A}$ beam of unpolarized, $1.1 \mathrm{GeV}$ electrons entered experimental Hall A after just one pass through the linear accelerators.

\subsubsection{The Beamline}

Upon entering Hall A, the electron beam is guided in a steel vacuum sealed tube toward the reaction point. Several devices are stationed along this beamline to monitor the qualities and position of the beam. Some of the measurements are destructive to the integrity of the 


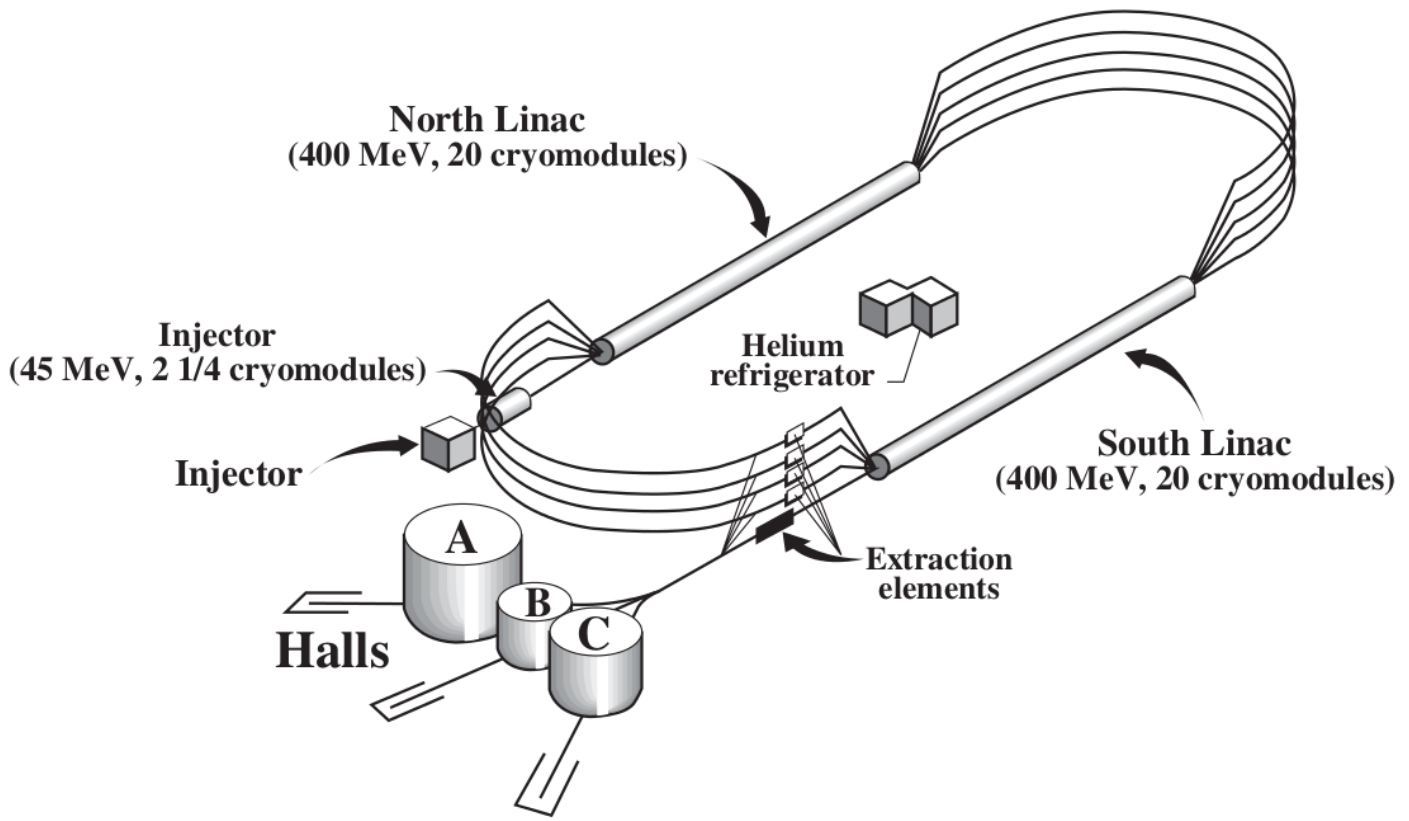

Figure 3.1: Overview of the JLab facility layout. Figure from [65].

beam, and must be utilized between experiments, while others monitor continuously while experimental data is being collected.

\subsubsection{Beam Position and Direction}

Absolute beam position is measured with two wire scanners (superharps) and two beam position monitors (BPMs). The superharps can locate the beam to within $200 \mu \mathrm{m}[65]$, and are calibrated during regular Hall A surveys. The BPMs record the relative position of the beam to within $100 \mu \mathrm{m}$, and their data is recorded in the EPICS data stream. Additionally, the beam is blurred to reduce localized heating effects. The raster for this experiment was a rectangular area approximately $2 \mathrm{~mm}$ by $3 \mathrm{~mm}$, centered roughly $5 \mathrm{~mm}$ from the target centerline, see Figure 3.2.

\subsubsection{Beam Energy}

The absolute energy of the electron beam is measured by either eP scattering in a detector $17 \mathrm{~m}$ upstream from the target, or measuring the radius of curvature in the recirculating arc of the CEBAF. Neither method can not be utilized during data runs, and are calibrated before the experiments begin. The Arc method results in uncertainty of $\Delta E / E$ of less than $1.2 \times 10^{-4}$, and the eP scattering results are better than $3 \times 10^{-4}$ [65]. 


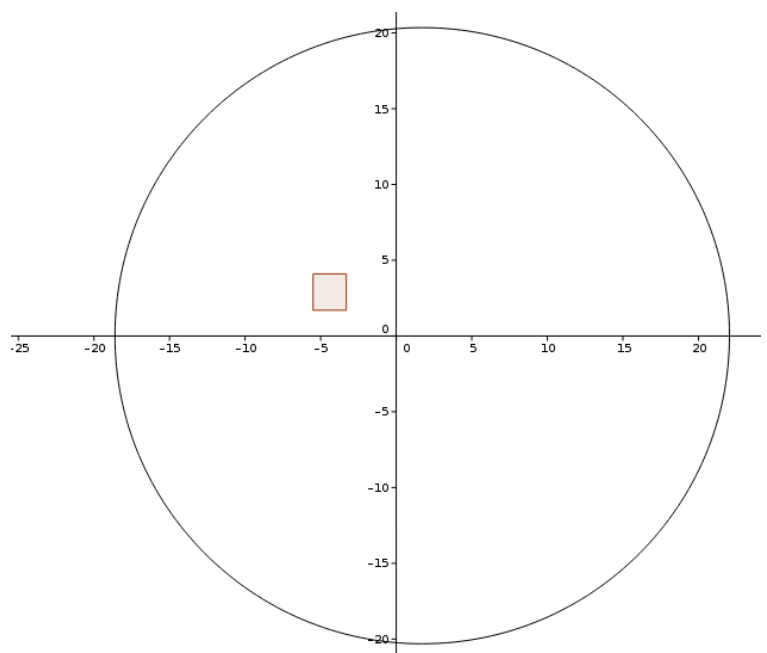

Figure 3.2: Target end cap view with beam position and raster shaded. Axes in mm.

\subsubsection{Beam Current}

The beam current and accumulated charge define the luminosity for the experimental cross section, and are measured by the same probes, which are calculated very carefully. There are three methods used to count passing charge. First, a Faraday Cup (FC) at the injector site of the CEBAF is used to provide a baseline during calibrations. The FC is very accurate, but destructive to the beam. No current reaches the experimental hall while the FC is in use. An Unser monitor and two RF cavities are located near the beamline entrance into Hall A, and were calibrated against the FC at the begining of the experiment. The Unser monitor is a Parametric Current Transformer providing an absolute measurement of the current. The value read out by the Unser monitor wanders slightly with a period of several minutes [65], so its value cannot be used for continuous measurements. For instantaneous beam current measurements, the two resonant RF cavities (tuned to the beam frequency at $1.497 \mathrm{GHz}$ ) provide a voltage output proportional to the charge passing in the beam. The signals from these beam current monitors (BCMs) are split and scaled to provide accuracy and prevent saturation across large current ranges. Half the signals are integrated to calculate charge passing by in consistent intervals. All together, six data streams from each BCM are stored in the scaler data stream every few seconds. In summary, careful calibration of the BCMs using the Faraday Cup and Unser monitor allows determination of the total luminosity to a temporal resolution of a few seconds and uncertainty better than $0.5 \%$. 


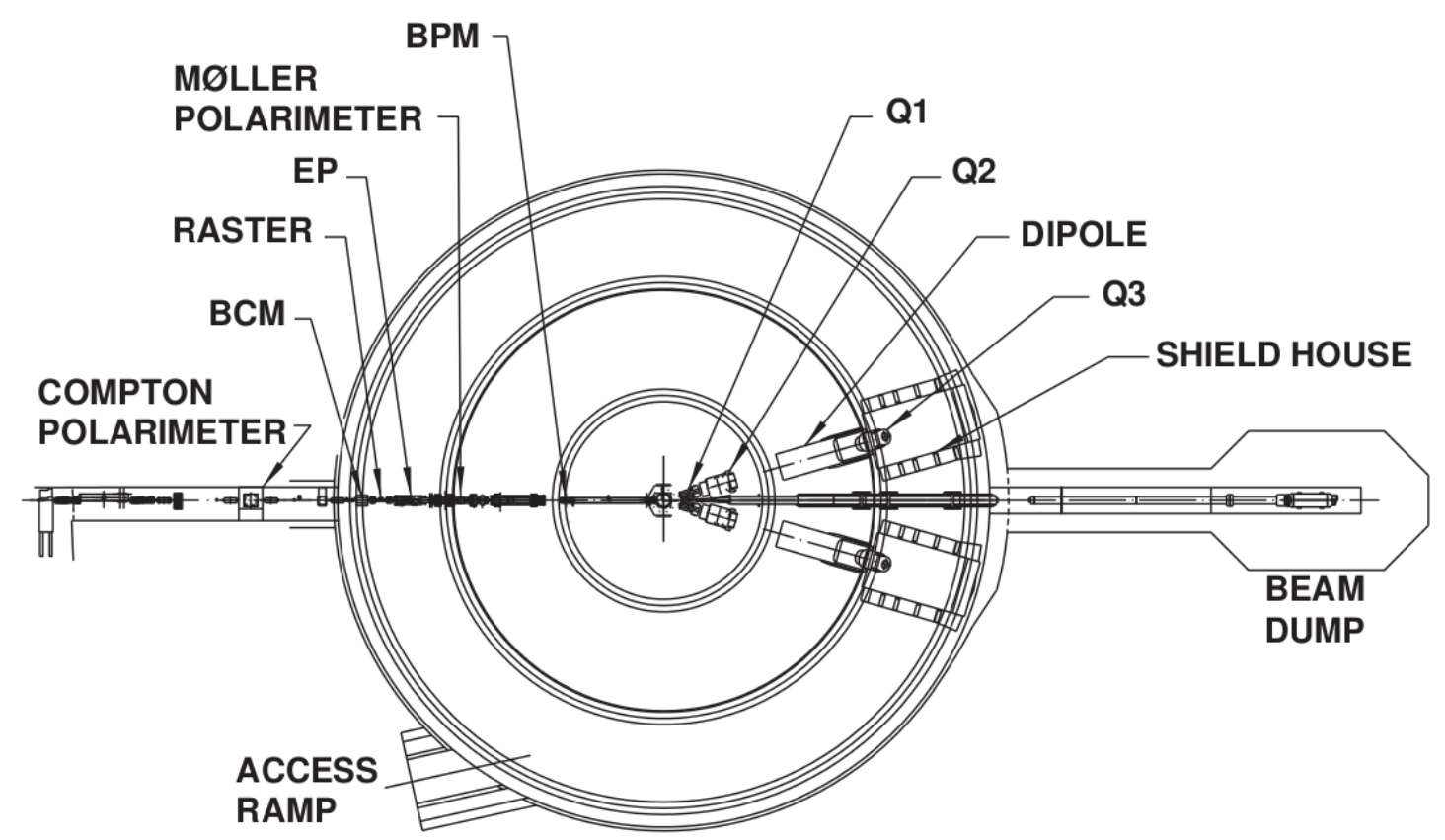

Figure 3.3: Overview of the Hall A beamline and beam monitors. Figure from [65].

\subsubsection{Beam Polarization}

The electron beam can be up to 85\% polarized by CEBAF[64]. Møller and Compton polarimeters are in the beamline to measure the polarization of the beam to 1\%. The Møller polarimeter requires scattering the beam off a magnetic foil, so it can not be used while collecting data. The Compton polarimeter measures the counting rate asymmetry of circularly polarized photons scattered off the electron beam, and is designed to monitor the beam polarization during data runs. The two polarimeters are cross calibrated to ensure proper monitoring[65].

\subsubsection{The Targets}

The Hall A laboratory is centered around a vacuum chamber holding a ladder of targets used in the experiments and calibration runs, as well as all of the ports and connections and equipment required to maintain their stability. The chamber is kept at pressures below $0.13 \mathrm{mPa}$, with the typical reading somewhat lower at $0.05 \mathrm{mPa}$ [66]. Two liquid hydrogen $\left(\mathrm{LH}_{2}\right)$ cryotargets of lengths $4 \mathrm{~cm}$ and $15 \mathrm{~cm}$ and $4 \mathrm{~cm}$ diameter were used for this experiment. Liquid deuterium ( $\left.\mathrm{LD}_{2}\right)$ cells of the same lengths were also present, but not used. Additional solid targets of carbon, beryllium oxide $(\mathrm{BeO})$ and aluminum were used 
for calibration purposes. Details of these targets can be found in the Table 3.1.

The cryogenic loops are cigar shaped tubes with length $4 \mathrm{~cm}$ and $15 \mathrm{~cm}$. Their lengths are regularly surveyed at room temperature between experiments. The effective length of the target differs from length contraction as the cryotargets are cooled, removing the thickness of the aluminum in the end caps, and also due to the beam offset and rastering on the curved surface of the end cap. The effect of thermal contraction is estimated to be $0.5 \%$ citationMeekins, $\mathrm{CRC}$ handbook. The metal in the end caps account for nearly 0.3 $\mathrm{mm}$, as seen in Table 3.1. Finally, the adjustment for end cap curvature is about $0.95 \mathrm{~mm}$, as calculated via Monte Carlo sampling using the measured beamline offset of $4.22 \pm 0.1$ $\mathrm{mm}$ in $\mathrm{x}$ and $2.74 \pm 0.1 \mathrm{~mm}$ in $\mathrm{y}$ and the raster of $2.19 \pm 0.1 \mathrm{~mm}$ in $\mathrm{x}$ and $2.36 \pm 0.1 \mathrm{~mm}$ in $y$ on the end cap of radium $20.32 \mathrm{~mm}$.

In summary, the effective target length is $14.8 \pm 0.02 \mathrm{~cm}$ for the $15 \mathrm{~cm}$ cell, and 3.86 $\pm 0.004 \mathrm{~cm}$ for the $4 \mathrm{~cm}$ cell.

Table 3.1: Summary of targets present during experiment. Thickness shown for the upstream, downstream, and side walls. Data from [67]

\begin{tabular}{|c|c|c|c|}
\hline Target Description & Upstream $\left(\mathrm{g} / \mathrm{cm}^{2}\right)$ & Downstream $\left(\mathrm{g} / \mathrm{cm}^{2}\right)$ & Wall $\left(\mathrm{g} / \mathrm{cm}^{2}\right)$ \\
\hline $15 \mathrm{~cm} \mathrm{LD}_{2}$ & $0.128 \pm 0.002$ & $0.194 \pm 0.009$ & - \\
\hline $4 \mathrm{~cm} \mathrm{LD}_{2}$ & $0.110 \pm 0.004$ & $0.147 \pm 0.008$ & $0.142 \pm 0.021$ \\
\hline $15 \mathrm{~cm} \mathrm{LH}_{2}$ & $0.081 \pm 0.004$ & $0.207 \pm 0.055$ & - \\
\hline $4 \mathrm{~cm} \mathrm{LH}_{2}$ & $0.145 \pm 0.004$ & $0.149 \pm 0.008$ & $0.141 \pm 0.014$ \\
\hline $15 \mathrm{~cm} \mathrm{Al} \mathrm{Dummy}$ & $0.275 \pm 0.003$ & $0.270 \pm 0.003$ & - \\
\hline $4 \mathrm{~cm} \mathrm{Al} \mathrm{Dummy}$ & $0.114 \pm 0.002$ & $0.116 \pm 0.002$ & - \\
\hline $\mathrm{BeO}$ & $0.149 \pm 0.001$ & - & - \\
\hline Carbon - Solid & $0.8918 \pm 0.0008$ & - & - \\
\hline Carbon - Slanted & $0.0419 \pm 0.0005$ & - & - \\
\hline $\mathrm{C}-(13$ foils to $\pm 15 \mathrm{~cm})$ & $0.0248 \pm 0.001$ & - & - \\
\hline
\end{tabular}

\subsubsection{The Detectors}

Two of the standard Hall A High Resolution Spectrometers (HRSs) detectors were used in this experiment. These are a package of several combined detectors working in concert to measure the trajectory of scattered electrons and protons very accurately. Table 3.2 provides the performance summary of the HRS detectors. The spectrometers use a series of electromagnets (4 magnets of quadrupole, quadrupole, dipole, quadrupole configuration) 
to direct the scattered particles of interest out of the reaction plane and through a series of individual detectors designed to calculate particles' the angle and momentum.

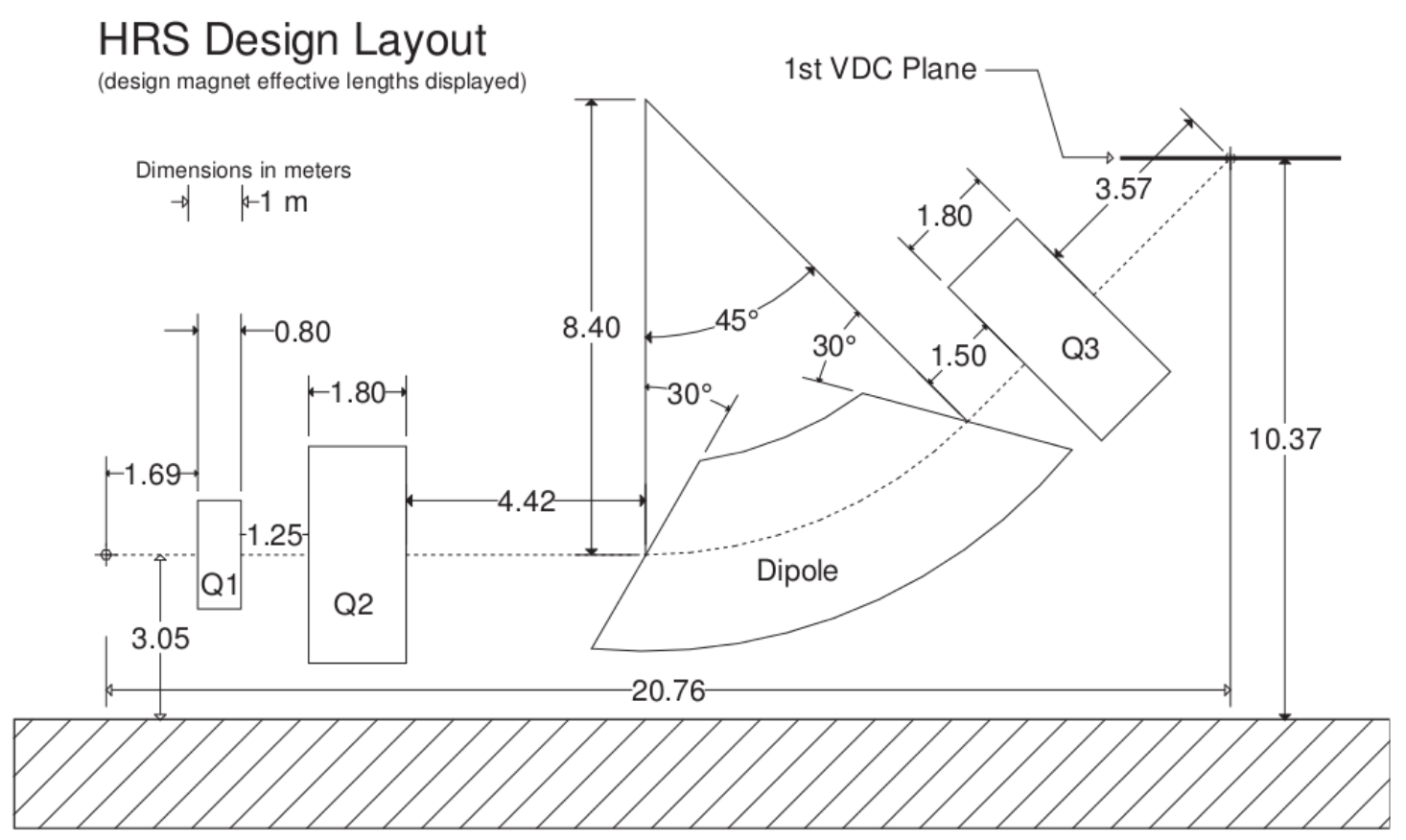

Figure 3.4: Diagram of HRS magnets. Figure from [65].

Table 3.2: Summary of HRS performance. Data from [65].

\begin{tabular}{|c|c|}
\hline Property & Value \\
\hline Momentum Range & $0.3-4.0 \mathrm{GeV} / \mathrm{c}$ \\
\hline Momentum Acceptance & $\left|\delta_{p} / p\right|<4.5 \%$ \\
\hline Momentum Resolution (FWHM) & $1 \times 10^{-4}$ \\
\hline Dispersion (D) & $12.4 \mathrm{~cm} / \%$ \\
\hline Horizontal Acceptance & $\pm 28 \mathrm{mrad}$ \\
\hline Vertical Acceptance & $\pm 60 \mathrm{mrad}$ \\
\hline Horizontal Resolution: (FWHM) & $0.6 \mathrm{mrad}$ \\
\hline Vertical Resolution: (FWHM) & $2.0 \mathrm{mrad}$ \\
\hline Transverse Length Acceptance & $\pm 5 \mathrm{~cm}$ \\
\hline Transverse Position Resolution (FWHM) & $1.5 \mathrm{~mm}$ \\
\hline Spectrometer Angle Determination Accuracy & $0.1 \mathrm{mrad}$ \\
\hline
\end{tabular}




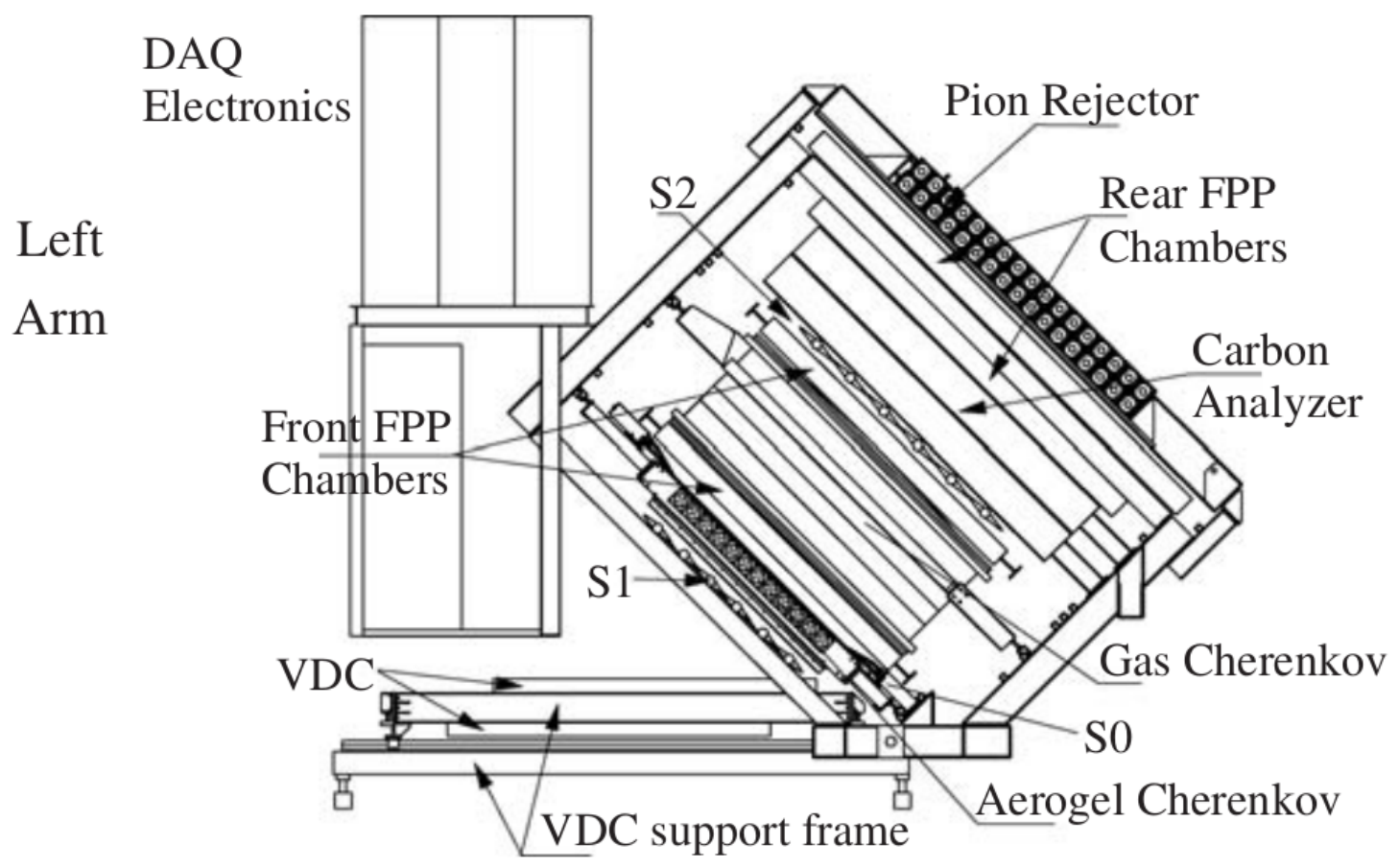

Figure 3.5: Overview of LHRS detector package. Figure from [65].

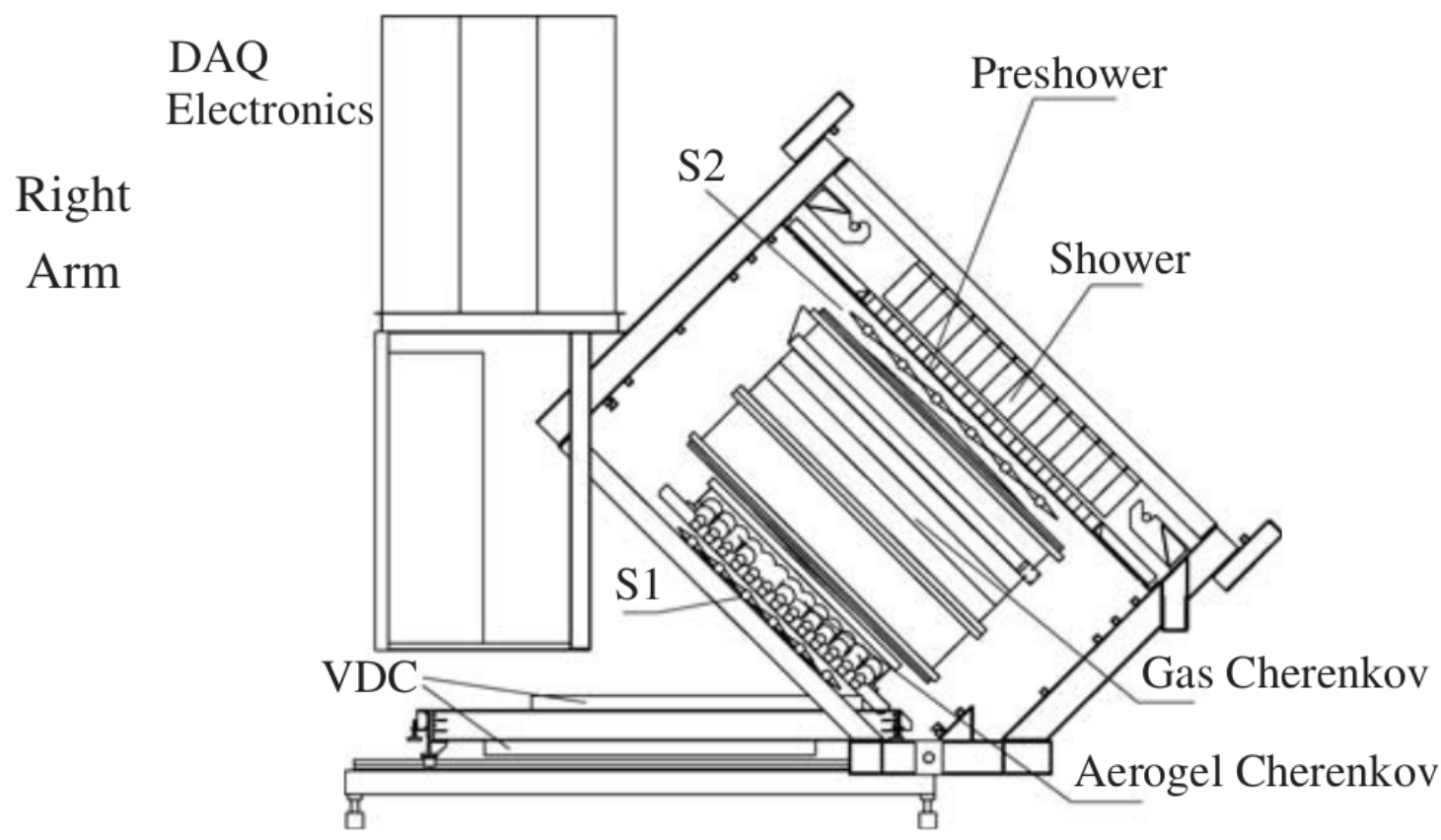

Figure 3.6: Diagram of HRS magnets. Figure from [65]. 


\subsubsection{Drift Chambers}

Preliminary tracking information comes from two vertical drift chambers (VDCs) in each HRS. Each VDC contains two planes of 368 tightly spaced parallel wires and is filled with a mixture of argon (62\%) and ethane (38\%) gas. The wires in each plane are $4.24 \mathrm{~mm}$ apart and kept at high voltage $(4 \mathrm{kV})$. The planes of wires are parallel, but the wires in each plane run perpendicular to the wires in the other. $26 \mathrm{~mm}$ separate the planes of wires within each VDC, and $335 \mathrm{~mm}$ separate the two VDCs. All of the planes are oriented such that the stream of detected particles makes an angle of 45 degrees to the normal vector [65].

Charged particles passing through the gas of the VDC cause a cascade of charged particles interacting with the high voltage wires creating a pulse of current in each wire that is correlated with the distance between the wire and the particle path. The orthogonality of the wires within each plane of the VDC allows a two dimensional reconstruction of the particle position within each VDC. $\chi^{2}$ fitting of the signal in each of the four planes identifies and scores a series of tracks through the detector for the rest of the analysis in each event.

Side view

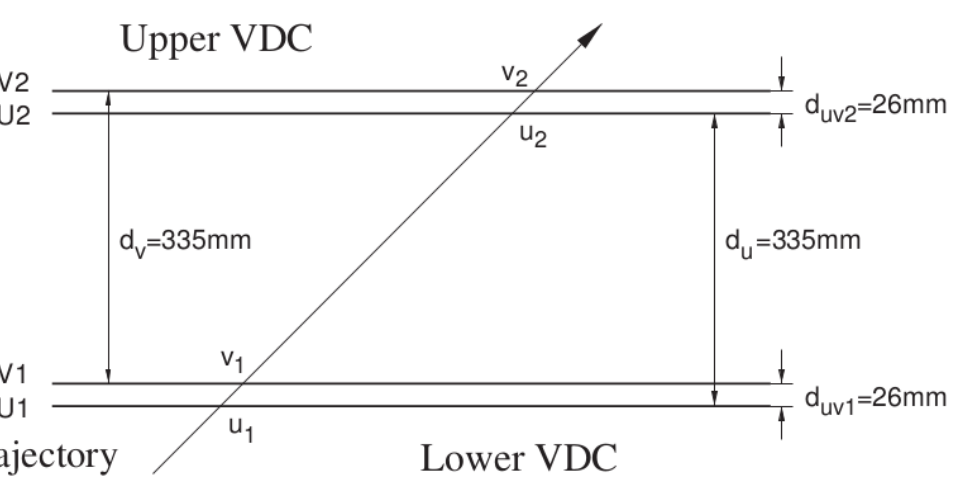

Figure 3.7: Diagram of VDC alignment within each HRS. Track reconstruction allows tagging of the data for particle momentum and number of particles entering the HRS. Figure from [65].

\subsubsection{Scintillating Panels}

The data acquisition trigger and timing information is provided by a series of three scintillating panels with two inch diameter Burle 8575 photomultiplier tube (PMT) detectors attached at both ends. These layers are labeled $\mathrm{S} 0, \mathrm{~S} 1$, and $\mathrm{S} 2 \mathrm{~m}$, and have slightly different constructions. S0 is a single bar of BICRON 408 plastic $10 \mathrm{~mm}$ thick and $170 \mathrm{~cm}$ long by 
$25 \mathrm{~cm}$ wide mounted with the short side $(25 \mathrm{~cm})$ parallel to the floor. The $\mathrm{S} 1$ plane has six $5 \mathrm{~mm}$ thick BICRON 408 panels that are $29.5 \mathrm{~cm}$ tall and $35.5 \mathrm{~cm}$ wide and mounted perpendicular to the S0 plane, with a $10 \mathrm{~mm}$ overlap between the bars. The $\mathrm{S} 2 \mathrm{~m}$ plane has been updated, and now consists of sixteen $5 \mathrm{~cm}$ thick panels of EJ-230 that are $14 \mathrm{~cm}$ by $43.2 \mathrm{~cm}$ and mounted with no overlap. Each bar has a timing resolution of roughly $0.3 \mathrm{~ns}$ citation [hrs].

The scintillator planes were used to define the trigger for data acquisition during the experiment. The single arm trigger T1 (T3) in the right (left) arm is defined by having the two PMTs at either end of a bar in both the S1 and S2m planes of the spectrometer (i.e. 2 matched pairs of PMTs) fire in coincidence. Should both a T1 and a T3 occur within the coincidence timing window, a T5 trigger is thrown and the event is stored for the final data stream. Additional triggers T2 (T4) are defined when a particle triggers a panel in the S0 plane of the right (left) HRS, but only one of S1 or S2m planes. The number of each type of trigger is stored, and the number of events triggering 2 of the planes, but not all three is used to calculate the efficiency of the triggering system.

\subsubsection{Cherenkov Detector}

The standard HRS package includes a $\mathrm{CO}_{2}$ Cherenkov detector between the $\mathrm{S} 1$ and $\mathrm{S} 2 \mathrm{~m}$ planes. Cherenkov detectors allows to discern particles based on the speed they are traveling through the gas of the detector. Particles entering the detector traveling faster than the speed of light in the gas will emit Cherenkov radiation an a manner analogous to an airplane breaking the sound barrier and emitting shock waves.

This detector was removed from the proton arm to prevent any losses from low energy protons being stopped in the $\mathrm{CO}_{2}$ before triggering the data acquisition defined with the $\mathrm{S} 2 \mathrm{~m}$ plane. Additionally, the Cherenkov was not found to be useful in the electron arm due to a high noise floor in the PMTs discovered during calibrations.

\subsubsection{Calorimeters}

The final subsystem in the HRS package is a detector made of two layers of lead-glass with PMTs mounted to each block. In the proton arm, these planes of blocks are labeled pion rejectors 1 and 2. In the electron arm, they are known as the pre-shower and shower calorimeters. Particles entering the blocks lose energy via bremsstrahlung and pair production, which are then subject to the same processes. This creates an electromagnetic cascade that is measured via PMTs behind the blocks. 
Each layer of the pion rejector contains two rows of $1714.7 \mathrm{~cm}$ square by $35 \mathrm{~cm}$ SF5 glass blocks. The two layers are offset so the second covers any gaps in the first. The preshower has two rows of 24 TF1 glass blocks, each $10.0 \mathrm{~cm}$ square by $35.0 \mathrm{~cm}$. 75 blocks make up the shower layer in five rows of 15 SF5 blocks, each $15 \mathrm{~cm}$ square by $32.5 \mathrm{~cm}$ [65].

Due to high noise levels in many PMTs, only the shower and PRL1 detectors are used for particle identification in this experiment.
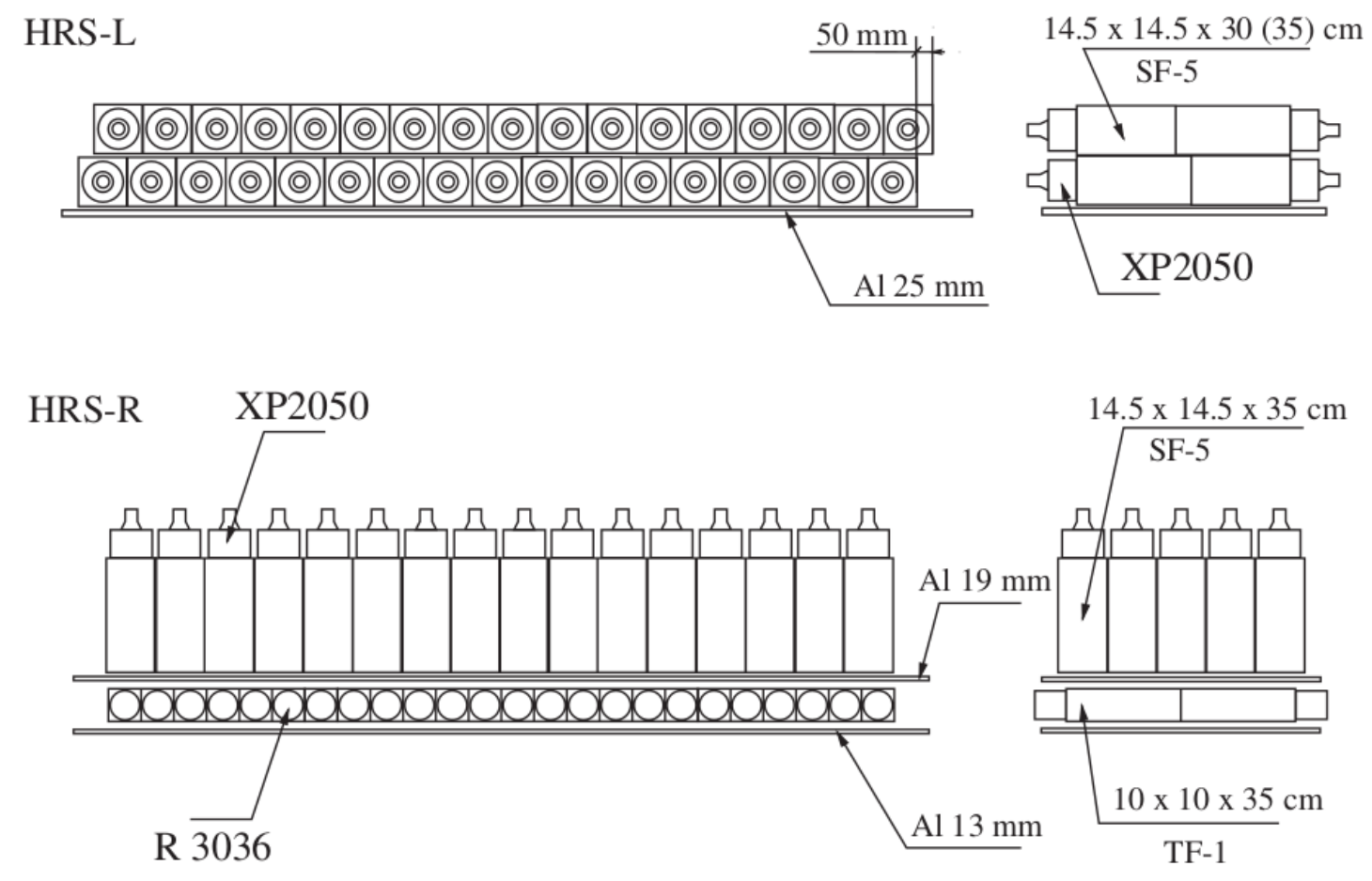

Figure 3.8: Diagram of PID calorimeters in each HRS. Note the careful offsets to ensure no particles pass without traversing the detector. Figure from [65].

\subsection{Mainz Microtron}

The experiment ran in September 10th to October 1st, 2012 at A1 Hall of the Institute for Nuclear Physics at the Johannes Gutenberg University in Mainz, Germany. Experiment A1/03-12, or "VCS-Delta" is part of a series of similar experiments to measure the generalized polarizabilities of the proton at small momentum transfer values. The hardware utilized in Mainz is very similar to that used at JLab. 


\subsubsection{The Electron Beam}

The electron accelerator facility in Mainz is the Mainz Microtron (MAMI). MAMI provides a continuous beam up to $1.5 \mathrm{GeV}$ at $100 \mu \mathrm{A}$. Polarized beams are achieved by changing the electron source. This experiment utilized an unpolarized beam of $1.1 \mathrm{GeV}$. Figure 3.9 provides an outline of the facility. The electron source is in the lower left of the diagram, with the beam continuing through the center of the facility and through to the experimental halls on the right, with A1 in particular at the lower right.

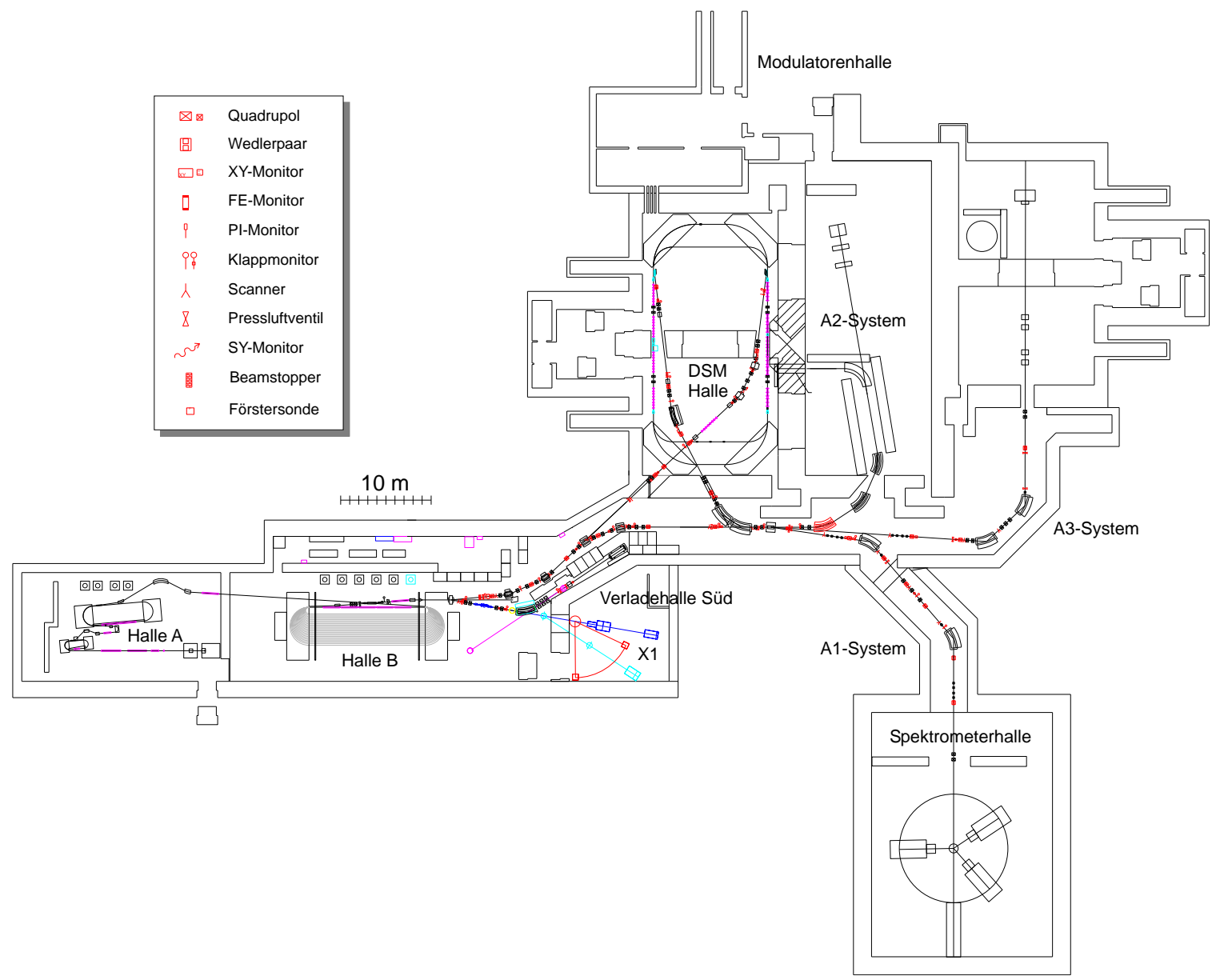

Figure 3.9: Layout of the Mainz Microtron. The electron source and RTMs 1-3 are in the lower right, the HDSM is in the center, and experimental halls are to the right. Figure from [68].

\subsubsection{The Beamline}

MAMI incorporates a set of four cascading accelerators to successively energize electrons in a continuous beam. The microtrons at MAMI have the same basic operating principle as 
the CEBAF at JLab, but accelerate the electrons by completing many more loops through slower acceleration linear accelerators as seen in Figure 3.10. Additionally, MAMI's final stage is a slightly different design, utilizing a harmonic double sided microtron (HDSM) with four dipole magnets that each bend the beam 90 degrees, rather than two dipole magnets affecting 180 degree turns as seen in Figure 3.11.

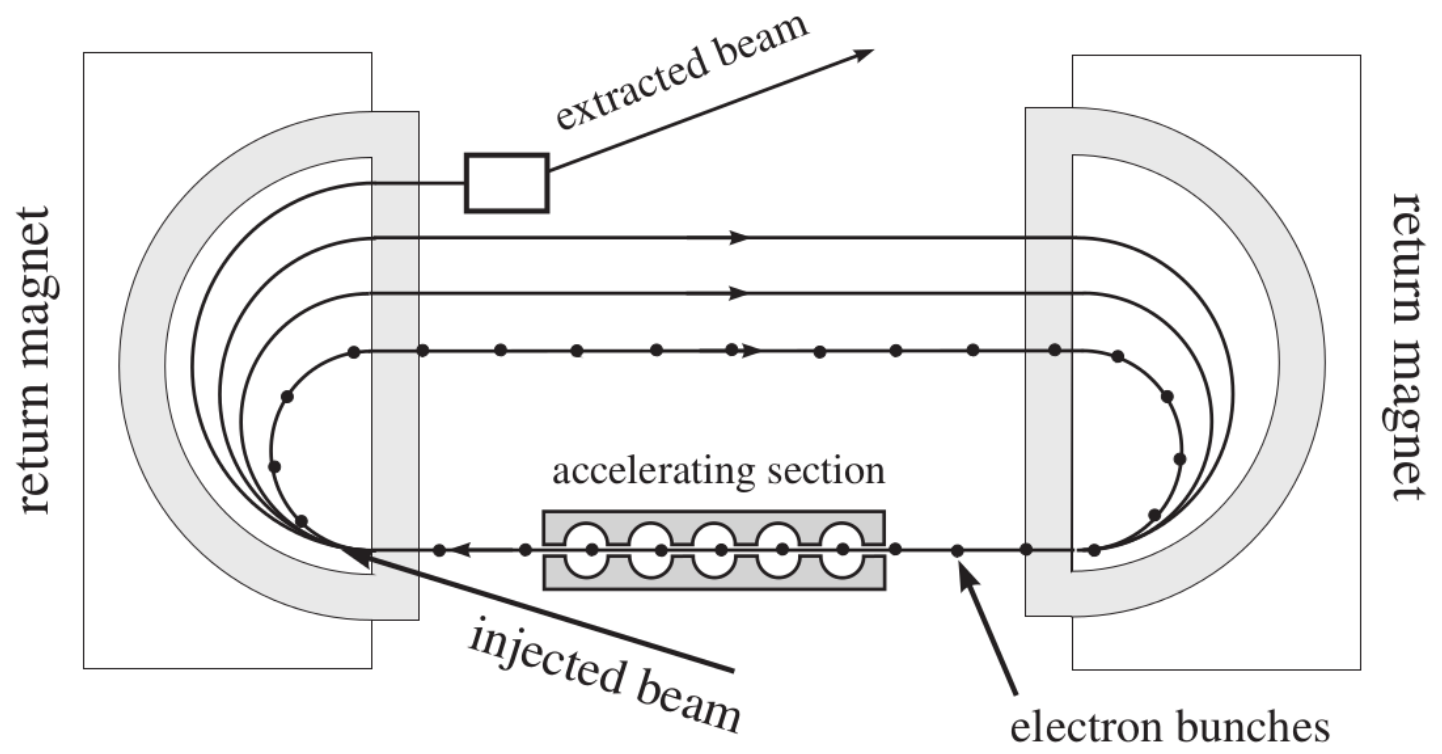

Figure 3.10: Overview of electron acceleration inside a RTM as seen at MAMI. Figure from [69].

MAMI begins with a $3.5 \mathrm{MeV}$ linear accelerator that feeds into a series of three racetrack microtrons (RTMs). The first RTM (RTM1) accelerates the beam to $15 \mathrm{MeV}$ over 18 recirculations, RTM2 takes the beam to $180 \mathrm{MeV}$ over 51 turns. Finally, RTM3 loops the beam 90 times accelerating to $855 \mathrm{MeV}$ before handing off to the harmonic double sided microtron (HDSM), which accelerates the beam to its final energy of $1.5 \mathrm{GeV}$ over 43 turns [68].

\subsubsection{Beam Position and Direction}

Unlike JLab, the beam position at MAMI A1 is not continuously monitored and stored in the data stream. Instead, the position and raster of the beam are manually checked during the day. This requires stopping production runs, changing to the $\mathrm{BeO}$ target, and capturing an image of the target cell using a dedicated camera. Figure 3.12 shows the typical rastered beam, centered on the target. The beam raster is a 3 by $3 \mathrm{~mm}$ square. 


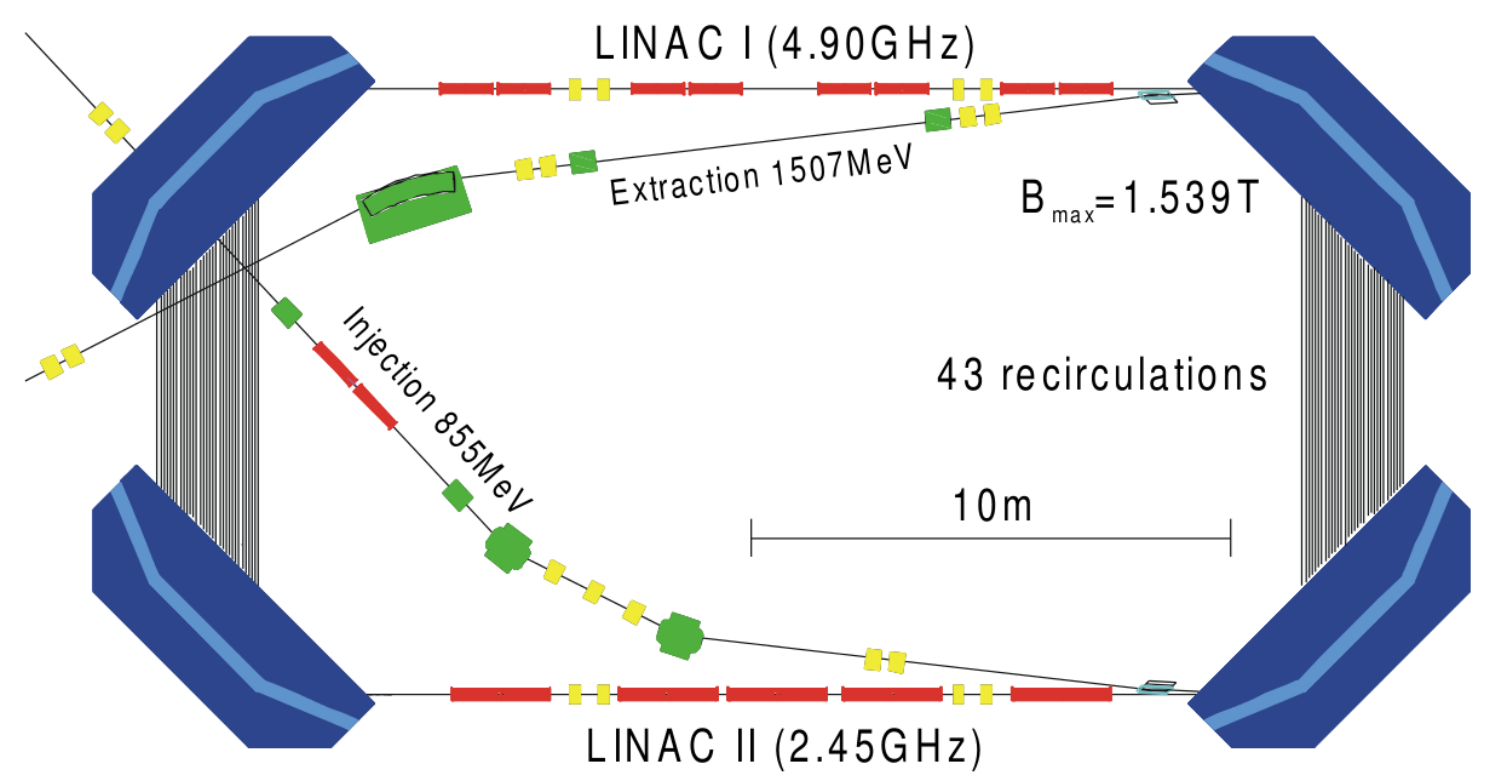

Figure 3.11: Overview of electron acceleration inside the HDSM at MAMI. Figure from [70].

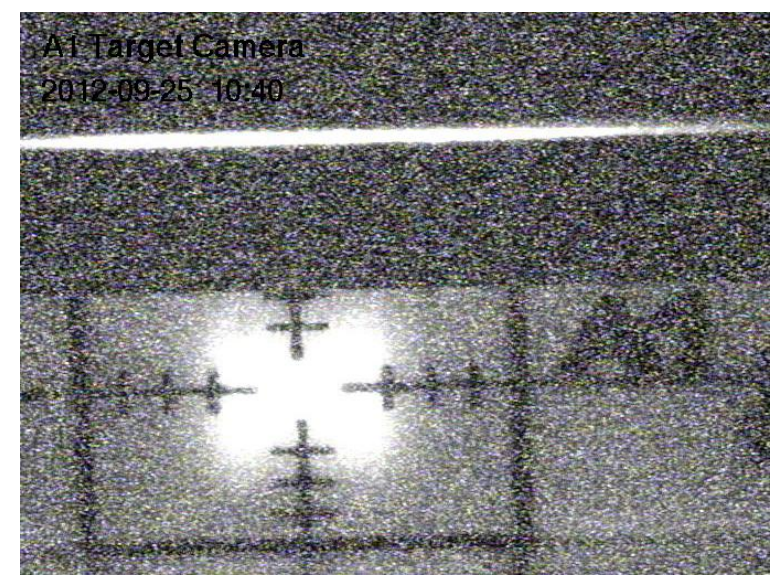

Figure 3.12: Image of the rastered beam on $\mathrm{BeO}$ target taken Sept. 25, 2012. 


\subsubsection{Beam Energy}

The beam energy at MAMI is carefully calibrated based on the number of turns through each microtron. The high degree of tuning required for resonance to accelerate the electrons repeatedly damps down fluctuations from RF phase and amplitude in the resonating cavities [71]. The energy fluctuations in the beam are dominated by synchrotron radiation effects at the $110 \mathrm{keV}$ level, or $1 \times 10^{-6}$ [68].

\subsubsection{Beam Current}

Beam curernt is monitored continuously by a Førster probe in RTM3. This measures the total current of all recirculations of the beam inside the microtron, which includes 90 recirculations [68]. The uncertainty of the Førster probe is $0.3 \mu \mathrm{A}$ [72], leading to a $<0.1 \%$ uncertainty in the beam current and luminosity.

\subsubsection{Beam Polarization}

Beam polarization at MAMI is achieved through the use of a polarized electron source. Typical polarizations of $75 \%$ are achieved with a strained Gallium-Arsenide (GaAsP) cathode [71]. The polarization is confirmed periodically in the hall with dedicated runs using a Møller polarimeter upstream of the target, similar to the one used at JLab. This experiment uses unpolarized electrons, so the Møller polarimeter and GaAsP source were not required.

\subsubsection{The Targets}

The target stack at MAMI A1 is much smaller than at JLab Hall A. A BeO target was used for basic beam positioning and optimization. A stack of three carbon foils was used to calibrate the optics matrix, and a liquid hydrogen cryotarget cell was used for the experimental production runs. The hydrogen cell is a tube $49.5 \mathrm{~mm}$ long and $10 \mathrm{~mm}$ in diameter. Rapid circulation of the hydrogen through the target cell coupled with a rastered beam prevent localized heating effects. Additionally, the beam current was kept below $20 \mu \mathrm{A}$, well under the $100 \mu \mathrm{A}$ design parameters of the target cell.

The target cooling system is powered by a $75 \mathrm{~W}$ Phillips-Sterling machine. The target was kept at $20 \mathrm{~K}$ and roughly 2 bar during the experiment, with the temperature and pressure recorded continuously into the data stream. The operating pressure of the cooling system was also monitored closely during the experiment to ensure the cooling system was stable, 
though this parameter was not stored. The beam current was tuned to keep the PhillipsSterling machine operating efficiently.

\subsubsection{The Detectors}

The MAMI A1 spectrometers are very similar to the HRSs used at JLab Hall A. The electromagnets used are optimized for different uses. Spectrometer A uses a complex quadrupolesextupole-dipole-dipole (QSDD) configuration to create a large acceptance in both solid angle and momentum. Spectrometer B is designed for use at small scattering angles with a single clamshell dipole magnet [71]. An added benefit of this design of Spectrometer B is increased sensitivity to the scattering position in the target. An overview of the spectrometers' properties can be found in Table 3.3, and Figures 3.13 and 3.14.

Table 3.3: Summary of A1 spectrometer performance. Data from [73]

\begin{tabular}{|c|c|c|c|}
\hline Property & Unit & Spec. A & Spec. B \\
\hline Maximum Momentum & $(\mathrm{MeV} / \mathrm{c})$ & 735 & 870 \\
\hline Momentum Acceptance & $\left|\delta_{p} / p\right|<(\%)$ & 20 & 15 \\
\hline Momentum Resolution (FWHM) & & $1 \times 10^{-4}$ & $1 \times 10^{-4}$ \\
\hline Dispersion (D) & $\mathrm{cm} / \%$ & 5.77 & 8.22 \\
\hline Horizontal Acceptance & $\mathrm{mrad}$ & \pm 35 & \pm 35 \\
\hline Vertical Acceptance & $\mathrm{mrad}$ & \pm 50 & \pm 10 \\
\hline Transverse Length Acceptance & $\mathrm{mm}$ & \pm 5 & \pm 5 \\
\hline Transverse Position Resolution & $\mathrm{mm}$ & $3-5$ & 1 \\
\hline Angular Resolution at Target & $\mathrm{mrad}$ & $<3$ & $<3$ \\
\hline
\end{tabular}

\subsubsection{Drift Chambers}

The VDCs at MAMI A1 operate on the same principle as those at JLab. High voltage wires are stretched across a cell containing a mixture of $80 \%$ ethane and $20 \%$ argon gas. There are two VDCs in each spectrometer, each containing two planes of alternating signal (15 $\mu \mathrm{m}$ thick) and potential (50 $\mu \mathrm{m}$ thick) wires separated by $2.5 \mathrm{~mm}$ [71]. The two planes of wires are labeled $\mathrm{x}$ (oriented along the non-dispersive direction), and s (at a 40 degree angle). Summary statistics for the number of wires in each plane, and the size of the sensitive area are shown in Table 3.4. The VDC chambers are set up so the central particle trajectory passes through at a 45 degree inclination. 


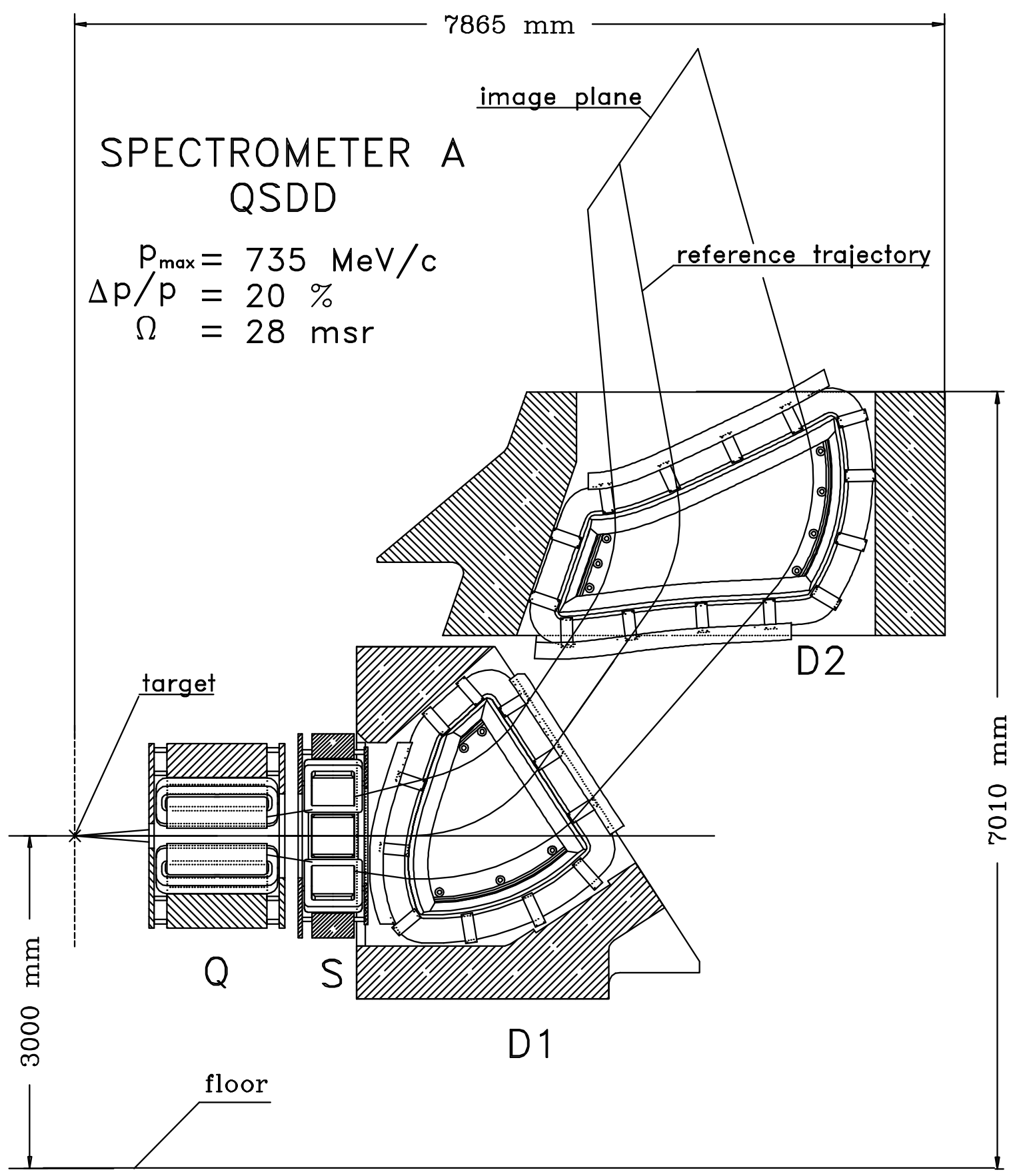

Figure 3.13: Electromagnet layout of MAMI A1 Spectrometer A. Figure from [73]. 


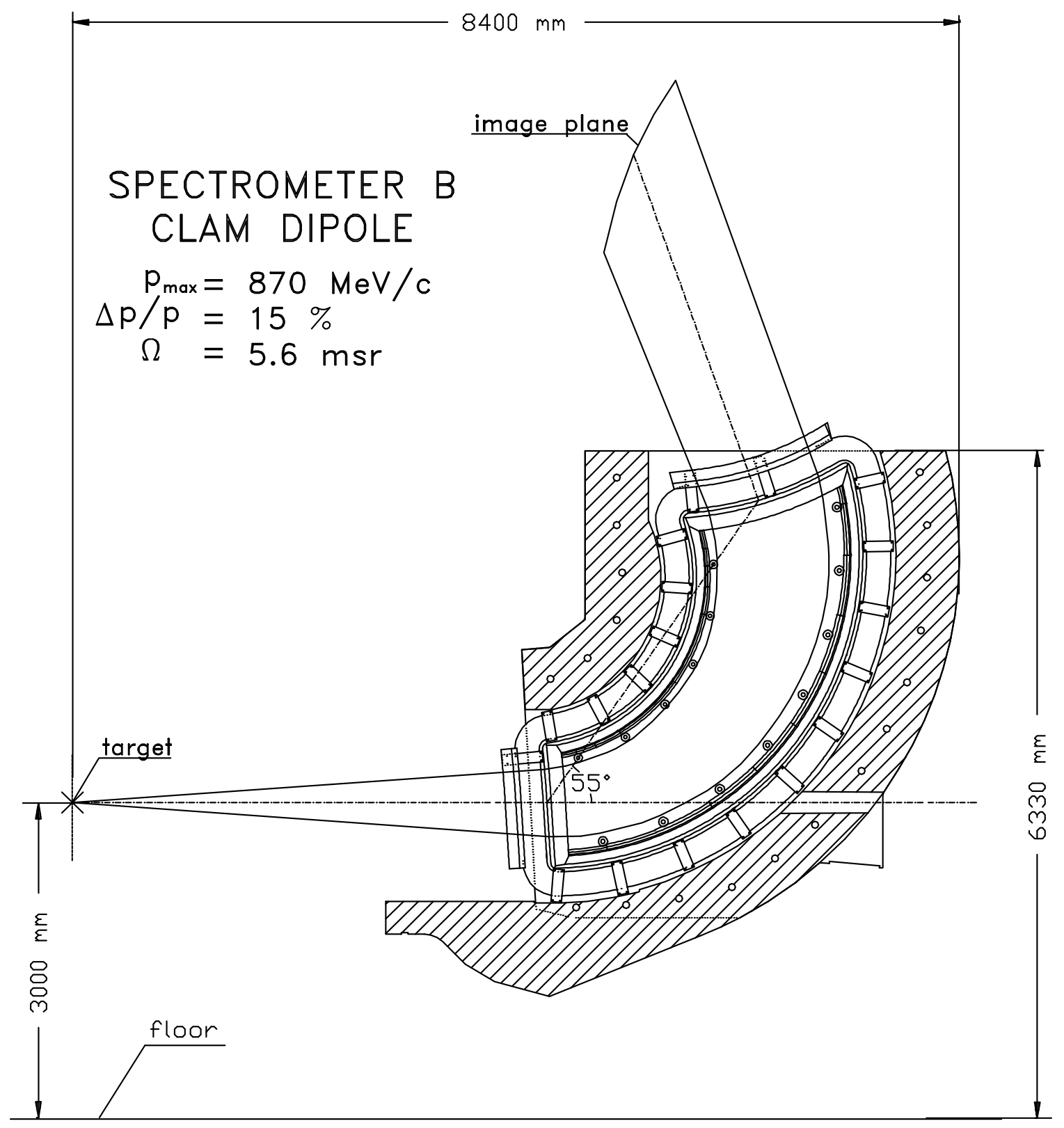

Figure 3.14: Electromagnet layout of MAMI A1 Spectrometer B. Figure from [73]. 
Table 3.4: Summary of VDCs in A1 spectrometers. Data from [71]

\begin{tabular}{|c|c|c|}
\hline Property & Spec. A & Spec. B \\
\hline Sensitive length $(\mathrm{cm})$ & 222 & 235 \\
\hline Sensitive width $(\mathrm{cm})$ & 40.5 & 12 \\
\hline x1 channels & 400 & 368 \\
\hline s1 channels & 320 & 336 \\
\hline x2 channels & 416 & 400 \\
\hline s2 channels & 336 & 368 \\
\hline
\end{tabular}

\subsubsection{Scintillating Panels}

Each Spectrometer contains two layers of scintillating material that provide timing and energy deposition information for each event. The first layer along the particle trajectory,

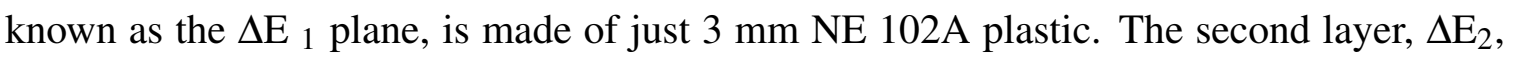
gives a faster timing response and is made from $10 \mathrm{~mm}$ of NE Pilot $\mathrm{U}$ plastic. All of the panels are 45 by $16 \mathrm{~cm}$ in spectrometer $\mathrm{A}$ and 14 by $16 \mathrm{~cm}$ in spectometer $\mathrm{B}$. The $\Delta \mathrm{E}_{1}$ panels have XP2262B PMTs at either end for data aquisition, while the $\Delta \mathrm{E}_{2}$ plane utilizes XP4222B PMTs on one side only for faster read outs [71].

\subsubsection{Cherenkov Detector}

Particle identification is achieved through a Cherenkov Detector in the spectrometer. The gas chamber is filled with $\left(\mathrm{CF}_{2} \mathrm{Cl}\right)_{2}$ (Freon 114) at atmospheric pressure, providing a cut off energy of $10 \mathrm{MeV}$ for electrons and $2.7 \mathrm{GeV}$ for pions [71]. The Cherenkov radiation is reflected off $40 \mathrm{~cm}$ by $50 \mathrm{~cm}$ spherical mirrors with radius of curvature $80 \mathrm{~cm}$ to highgain Philips XP4500B PMTs. Figure 3.15 shows the full detector package for the A1 spectrometers, with the Cherenkov Detector at the top. 


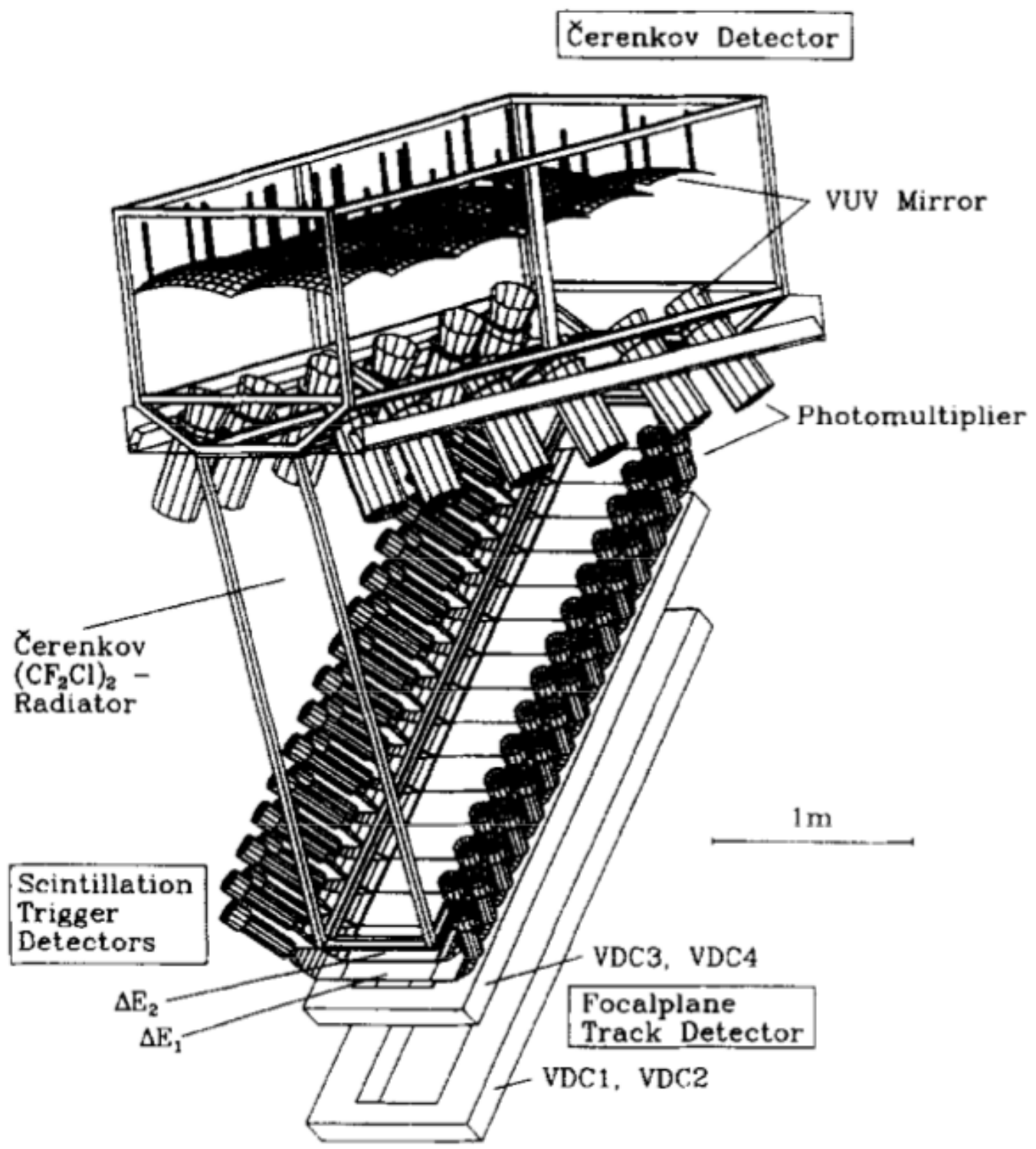

Figure 3.15: Detector package from A1 spectrometers. Figure from [71]. 


\section{CHAPTER 4}

\section{CALIBRATIONS}

\subsection{Beam Current}

As described in the previous chapter, the beam at JLab is monitored via three independent detector systems. The Faraday cup intercepts the total beam before entering the Hall, and can not be used during experiments. Instead, it is used to calibrate resonant cavity monitors near the injection site. These RF cavities are then used to calibrate the RF cavity BCMs in Hall A.

Figure 4.1 shows the relationship between the Faraday cup current $(I(F C))$ and the current in the RF cavities at the injection site $(I(0 L 02))$. The data was collected March 3rd, 2011, just after the experiment, and Fitting was performed in [58] finding the relationship $I(F C=[0.999 \pm 0.012] I(0 L 02) \mu A$ when averaged over the current test runs. The Hall A BCMS are then calibrated to the 0L02 monitor with a similar fit for each channel.

Figure 4.2 shows how the data was collected. First the FC is inserted in the beam and the data is measured at high current in both the FC and the RF cavities at the injector. The beam is then stepped down to zero over a series of progressions. In this case the FC is moved in and out of the beamline to show the Hall A beam current monitors (BCMs) collecting data in step with the other detectors.

At Mainz, the MAMI operators regularly calibrate the beam parameters in a similar fashion. The current is not measured directly in the hall, so there is no specific calibration performed at the A1 hall. 


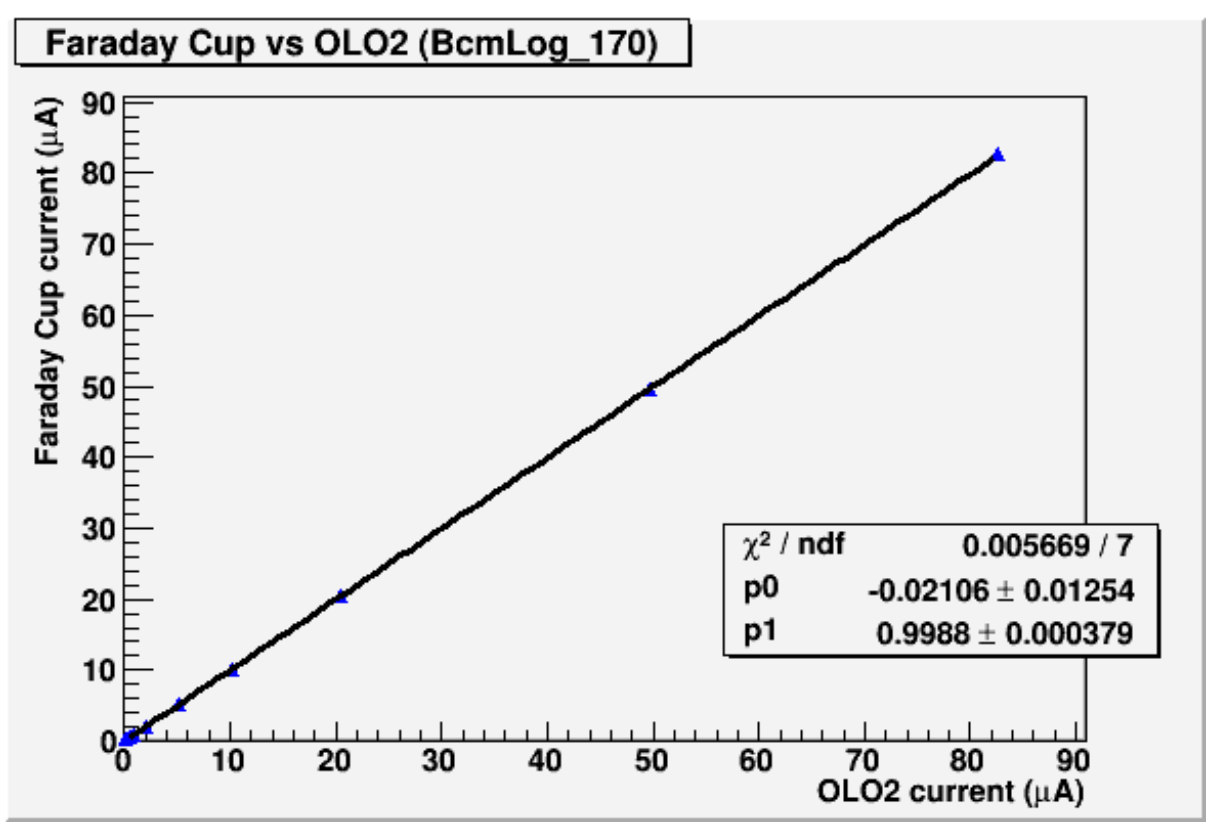

Figure 4.1: Calibration run of the beam current detectors near the CEBAF injection site. OL02 is an RF cavity current monitor. Figure from [58].

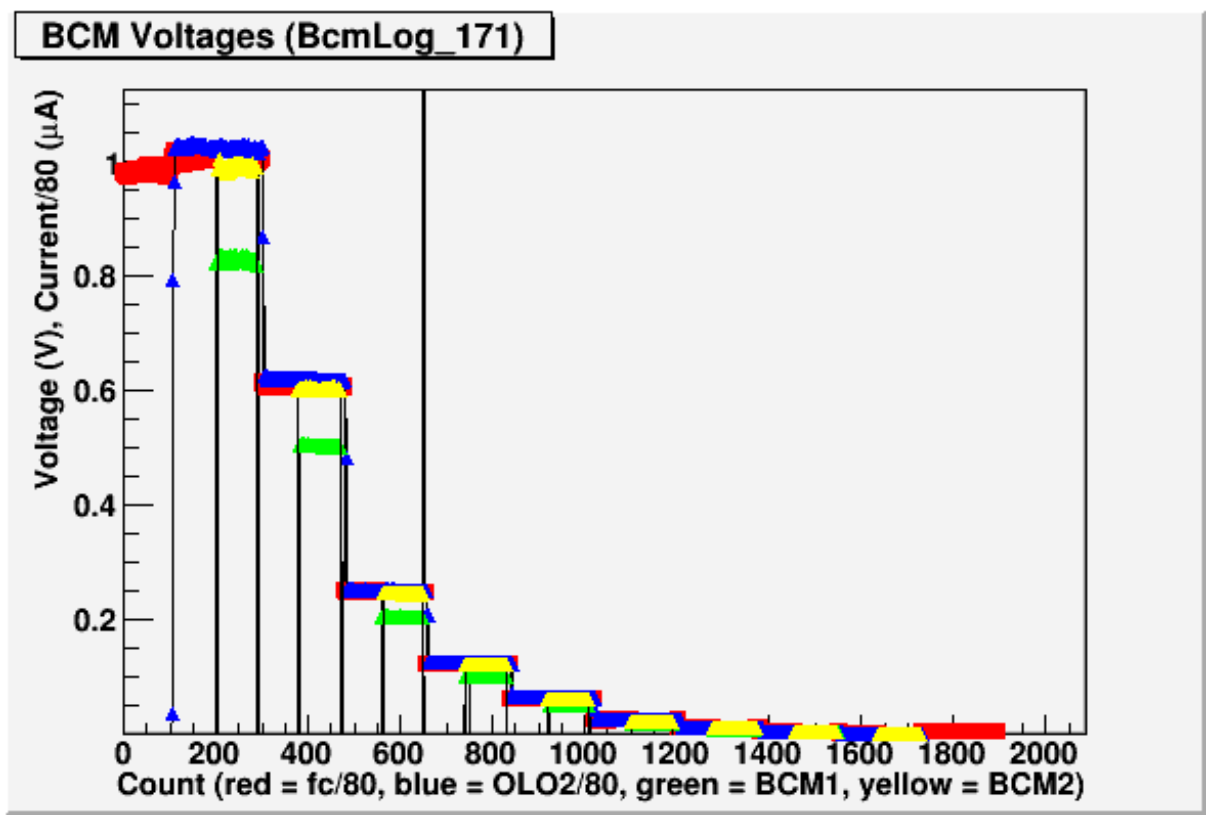

Figure 4.2: Sample run showing voltage from four beam current monitors at JLab. fc and 0L02 (red and blue) are current monitors at the injector site, and the BCMs (green and yellow) are near the beamline entrance to Hall A. Figure from [58]. 


\subsection{Beam Position}

The beam position at JLab is carefully calibrated and controlled through the CEBAF. The known position is stored periodically in the data stream via the Experimental Physics and Industrial Control System, (EPICS), but not for every event. The Hall A beam position monitors (BPMs) measure the beam position in two dimensions for every event, but need to be calibrated to lab coordinates to match the EPICS data. This is done by taking a series of runs with the beam in a known, but varied position. A linear transformation transformation converts the BPM data to match the known position from the EPICS.

Figure 4.3 shows the test run positions from the BPM and the EPICS data. Each test position is labeled to match up between the upper and lower plots. Figure 4.4 shows the result of the calibrations performed by [58]. The slope of the calibrated BPM vs EPICS data fit is 1 with small errors, indicating good agreement between the two beam position monitoring systems.
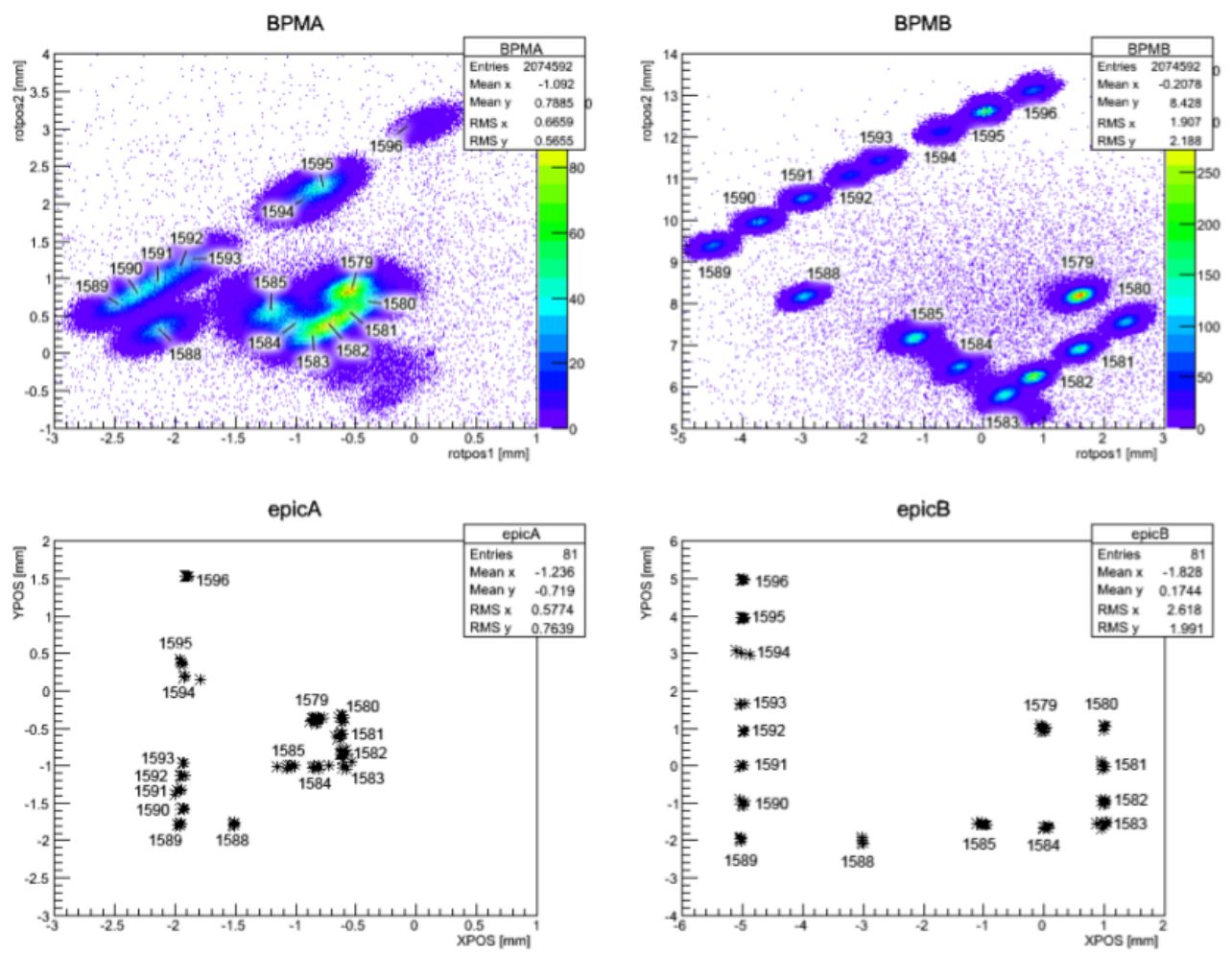

Figure 4.3: Top: Hall A BPM data from test runs with fixed beam positions (see labels). Bottom: CEBAF EPICS data from same runs, the different labeled positions show the need to calibrate the linear transformation matrix before using BPM data. Figure from [58]. 

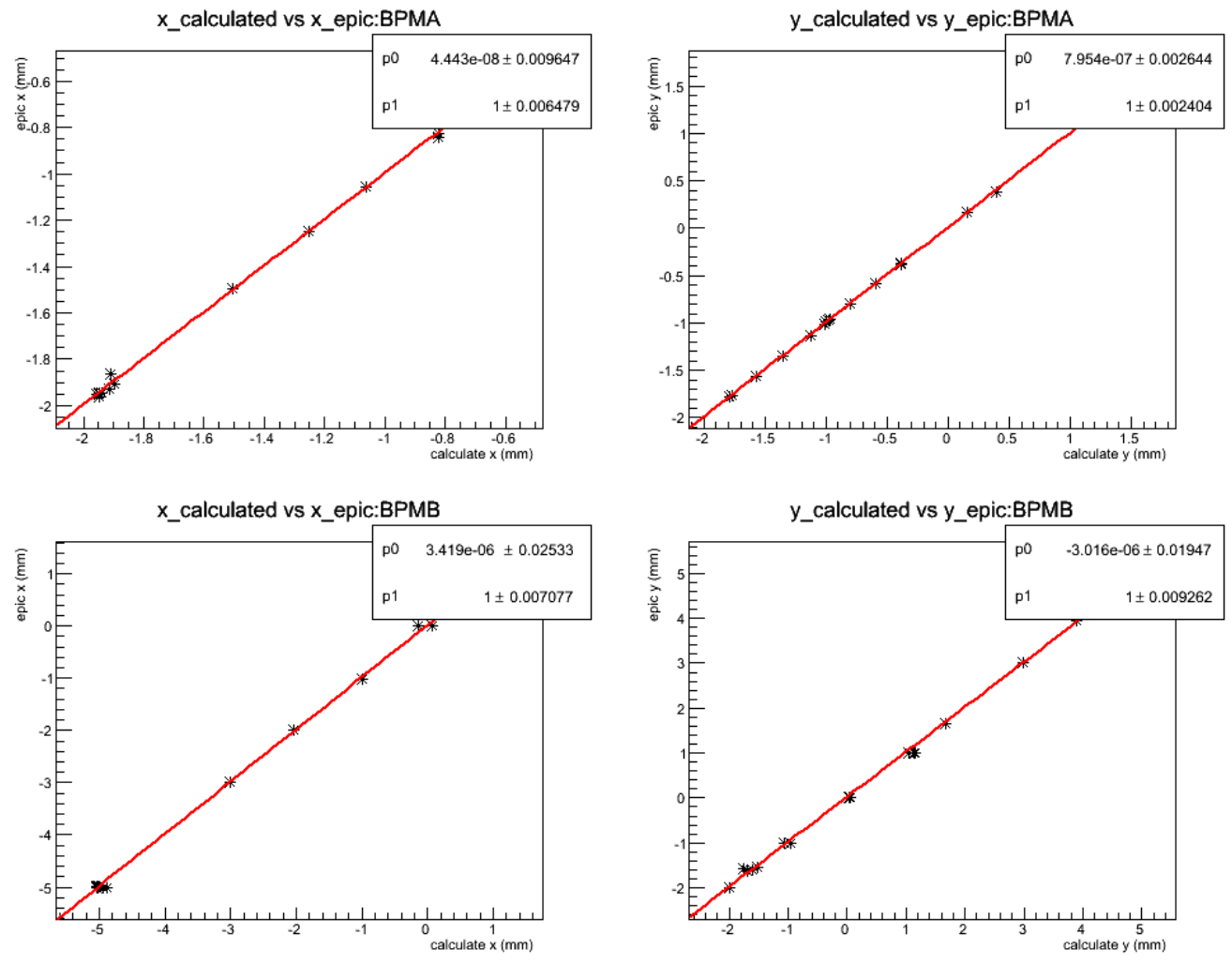

Figure 4.4: Test of the BPM calibration. The position read from the Hall A BPMs is plotted vs the EPICS positions. A linear fit shows good agreement after calibration. Figure from [58]. 
The MAMI beam position is not measured with a dedicated detector to be stored with each event. The rastered beam is positioned on a test target, which is photographed periodically throughout the experiment to ensure there is no drifting or change in beam shape. Figure 3.12 shows an typical position check image. Where the reaction position is required in $\mathrm{X}$ and $\mathrm{Y}$, the spectrometer track reconstruction is used.

\subsection{Vertical Drift Chambers}

The vertical drift chambers (VDCs) provide tracking information in the spectrometer. Each wire fires when a particle passes nearby and the timing information between wires is used to infer position and direction through the chamber. As such, it is crucial that the wire response is understood, and that the relative timing between wires is accurate. For this, the timing information from each wire is read out, and the timing for the closest events is aligned between all the wires. Figure 4.5 shows a typical VDC timing spectrum for all the wires in the LHRS V1 chamber before calibration. The ragged right edge indicates the timing offsets between wires. Figure 4.6 shows the same spectrum after calibration by [58]. Note the peak position is reversed in Figure 4.6 since the peaks occur due to tracks passing extremely close to the wire in question, indicating small response times.

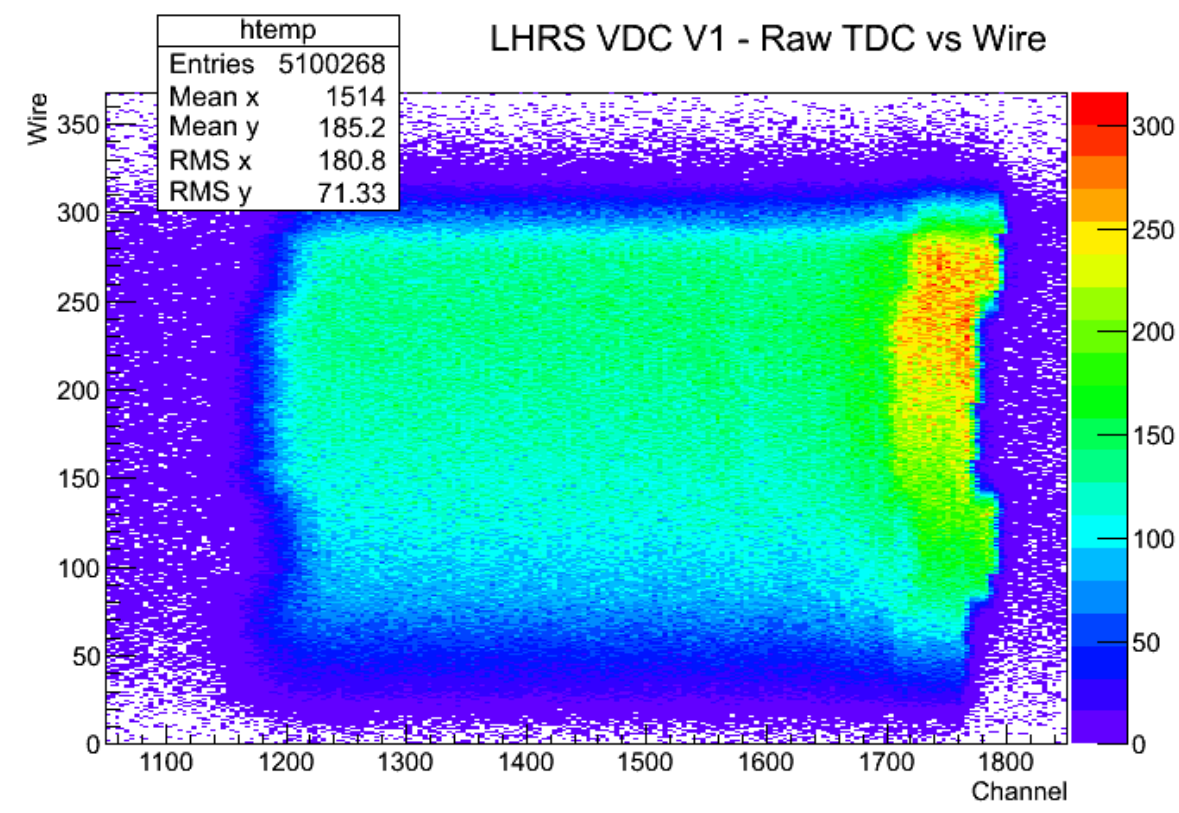

Figure 4.5: VDC wire timing spectrum before calibration. Figure from [58]. 


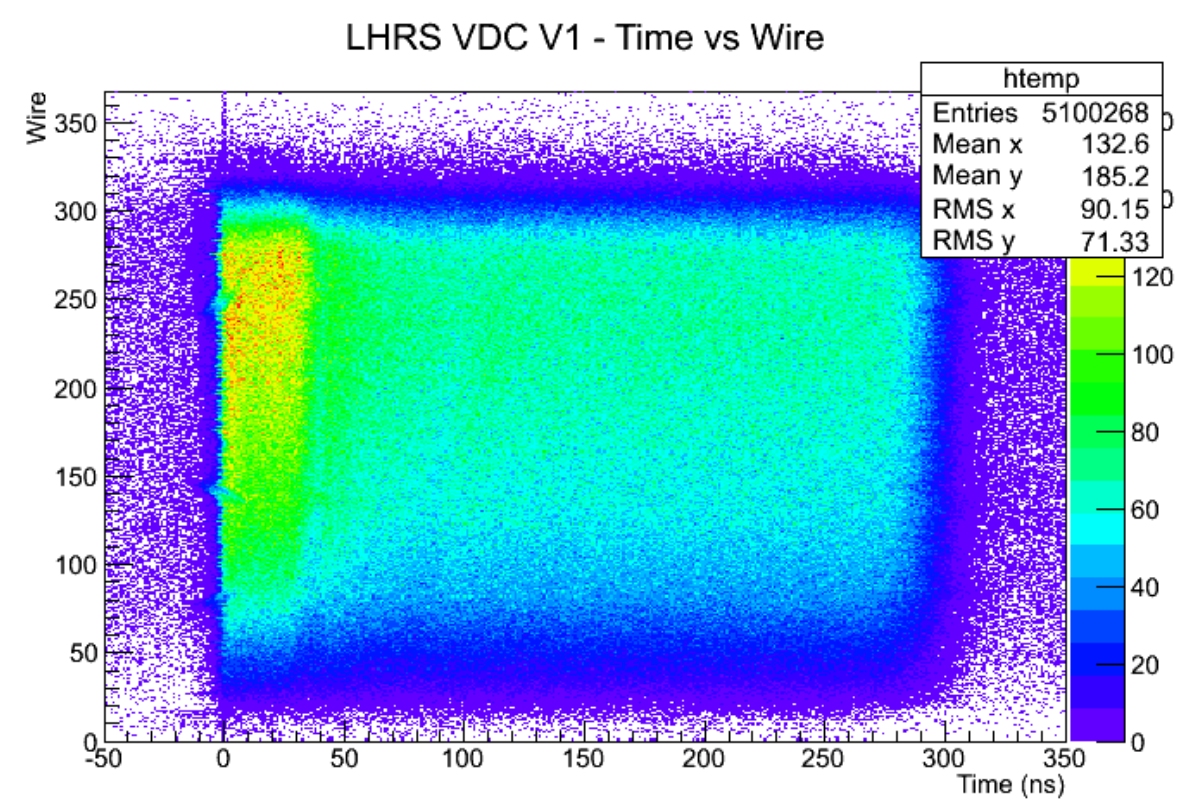

Figure 4.6: VDC wire timing spectrum after calibration. Figure from [58].

\subsection{Optics Matrix}

Because the detectors at both JLab and MAMI are well understood, Optics matrices from previous experiments were used. Plots of the reconstructed reaction vertex position in the target are used to verify the reliability of the optics matrix. The Z-vertex in particular is useful when looking at calibration runs on thin foil targets with known positions. Figure4.7 shows an example of the foil spectrum from MAMI. For this experiment, Helene Fonveille provided optimized matrices that improved upon the A1 defaults. 


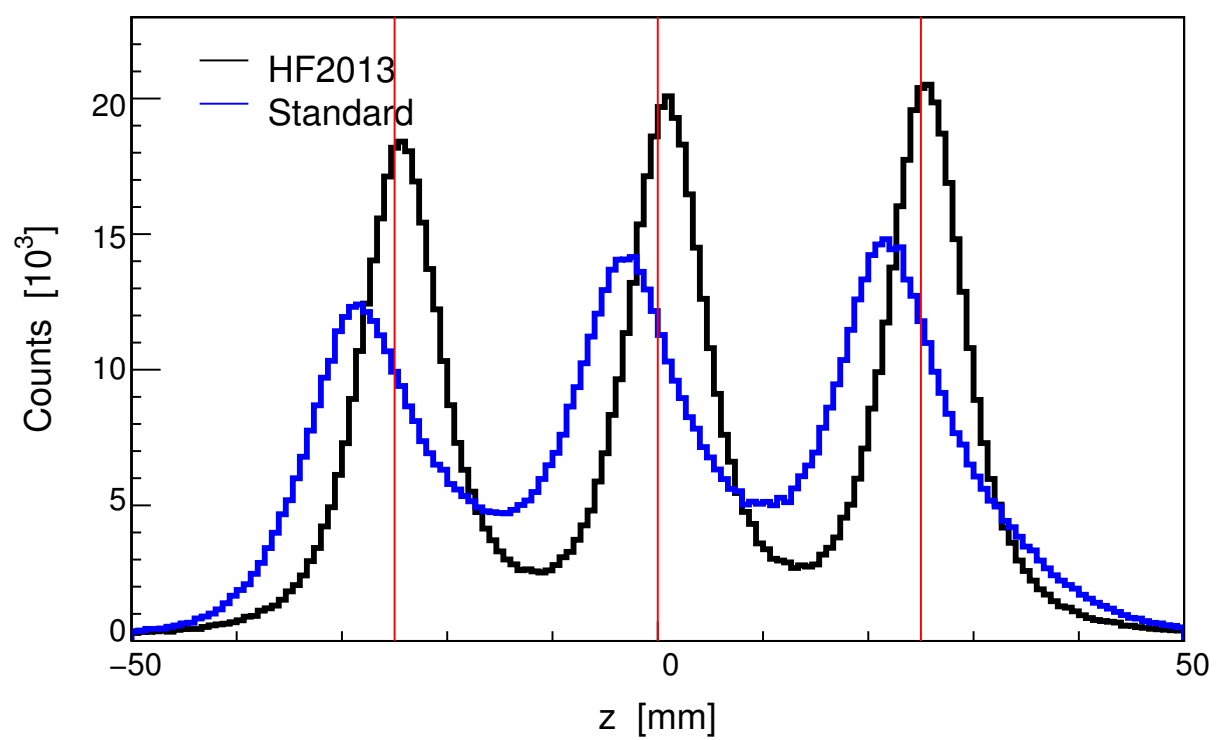

Figure 4.7: Comparison of the $\mathrm{z}$ vertex reconstruction using the standard optics matrices, and the optimized matrices from Helene Fonveille.

\subsection{Coincidence Timing}

Coincidence timing is crucial to these experiments, and has been performed many times before. In the case of MAMI, the site staff maintain the code to properly detect and calculated coincidences, and a simple plot indicating proper function was all that was required (see Figure4.8).

The experiment at JLab, however, suffered significant issues with the timing. The cable run lengths from each PMT in the scintillating bar detectors at JLab are manipulated to set the data collection triggers. This was not performed properly during our run time, and the trigger was not fixed for all events. Normally the signal from PMTs on one side of each spectrometer is defined as the trigger, and the cables are shortened relative to the other side to ensure their signal arrives at the DAQ first. This was not the case for the RHRS, where both sides had cable runs of the same length. As a result, the trigger was sometimes defined by the left set of PMTs on the $\mathrm{s} 2 \mathrm{~m}$ bars, and sometimes defined by the right side, leading to two peaks in the timing spectrum, as shown in Figure4.9. This issue was fixed by redefining the timing to a trigger that had a fixed reference. This was done to both arms, so the relative time difference between the arms would be unchanged by the analysis. More details are found in [58].

Figure 4.9 also demonstrates an alignment calibration required between each bar of the 


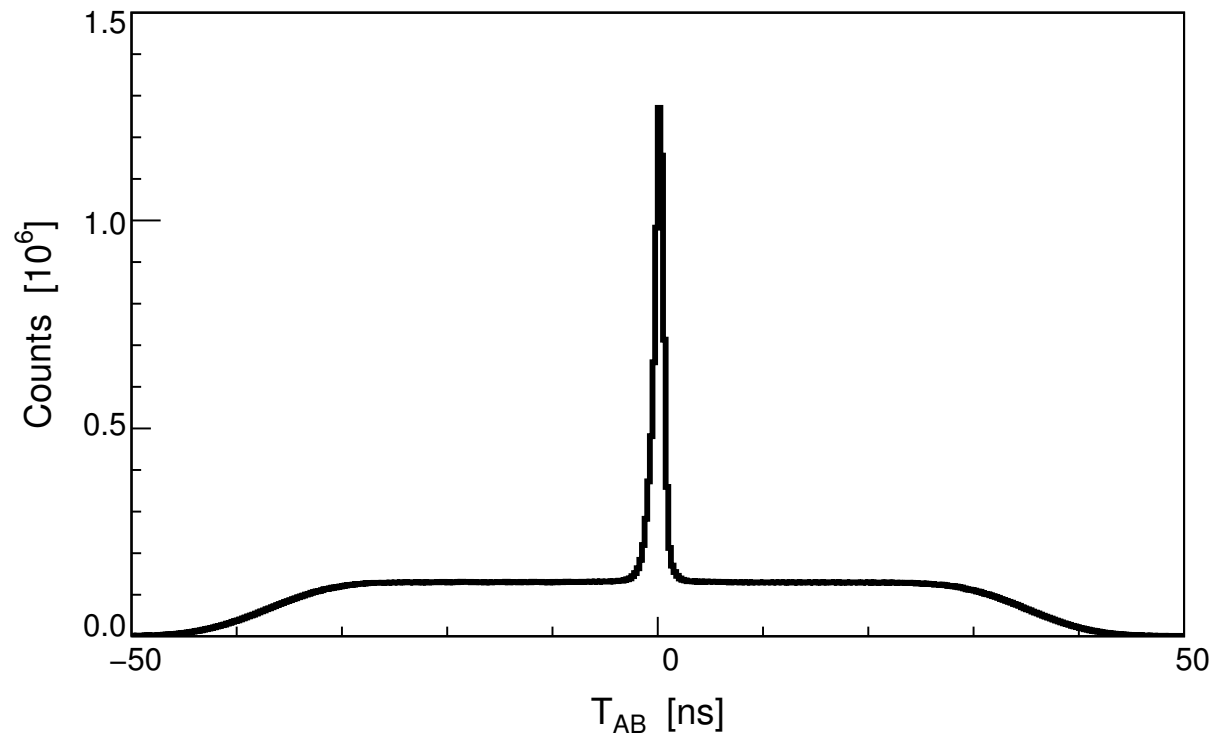

Figure 4.8: Coincidence timing plot from MAMI showing resolution comparable to design specifications.

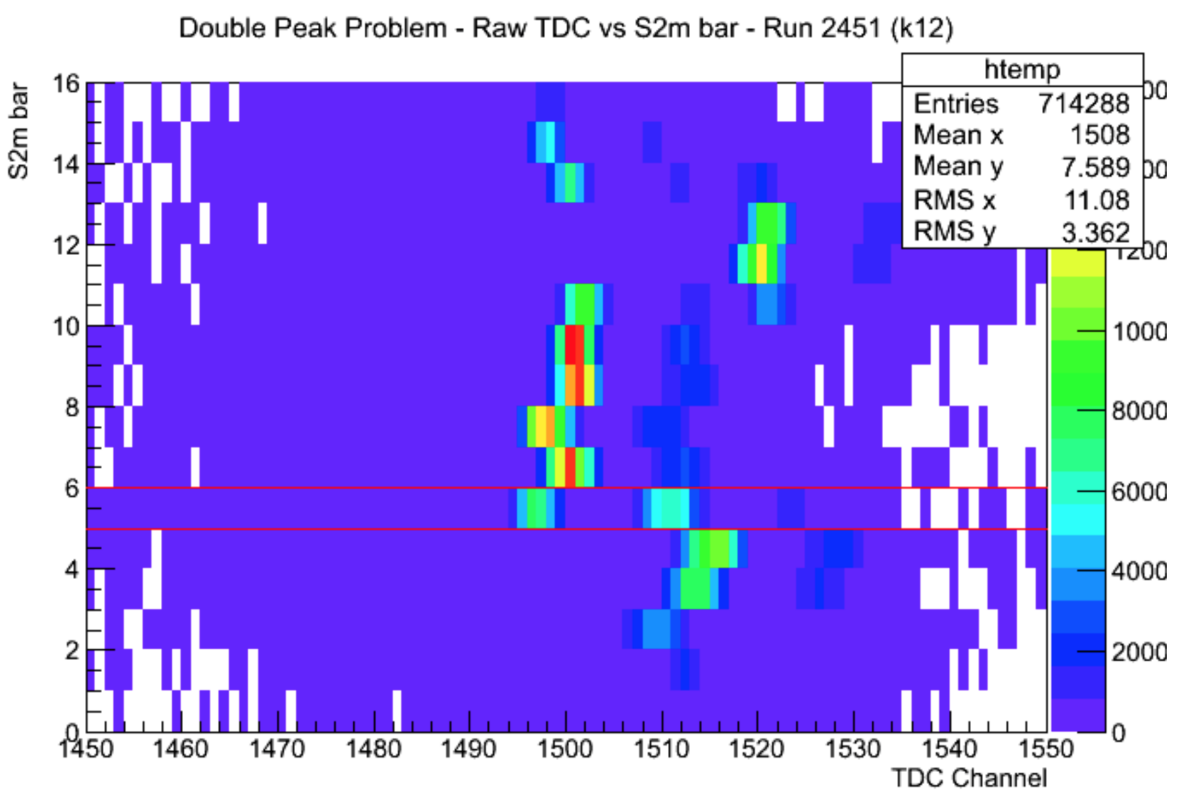

Figure 4.9: Double peak structure of the timing spectrum shown for each bar of the S2 scintillating plane. Bar 5, highlighted in red, shows the double peak from improper triggers. Figure from [58]. 
detectors. Simple offsets were applied (after the double peak issue was resolved) to align the timing from each bar.

The final corrections applied to the timing spectrum correct for correlations in the timing spectrum with respect to other detector variables, such as the momentum or position through the detector. These corrections account for the differences in path length traversed through the detector. Figure4.10 shows an example correction for time of flight vs. LHRS momentum, where a slight misalignment is fixed with the addition of a correction factor in the TOF that is quadratic in LHRS momentum.
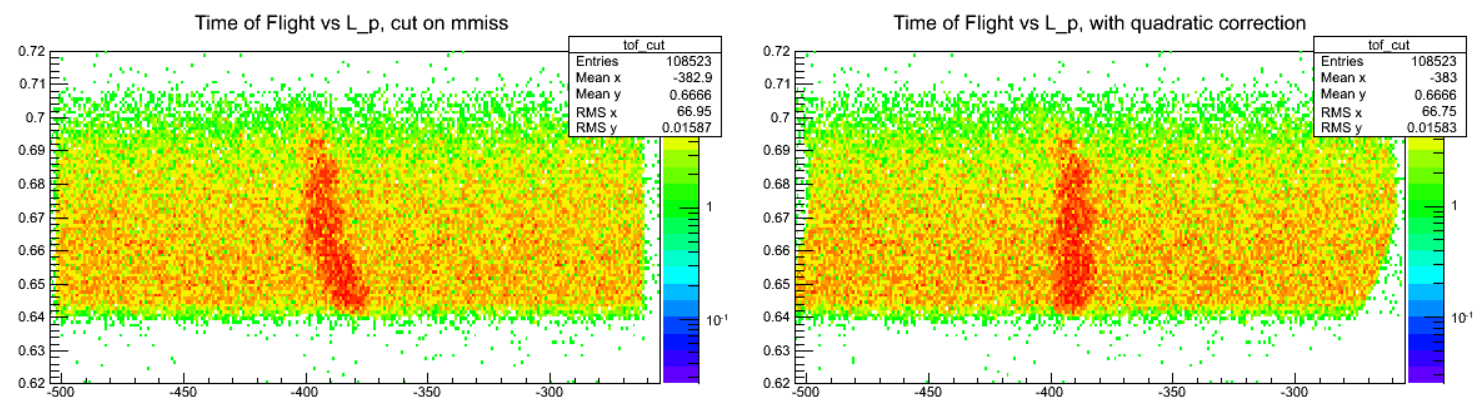

Figure 4.10: Correcting the coincidence time of flight for correlations with spectrometer variables. Shown is the lLHRS momentum vs coincidence time before (left) and after (right) a timing correction term quadratic in LHRS momentum.

The coincidence timing spectrum is shown in Figure 4.11 before (top right) and after calibrations. The calibration process increased the timing resolution by an order of magnitude. One can clearly see the RF structure of the electron beam in the final timing spectrum. 


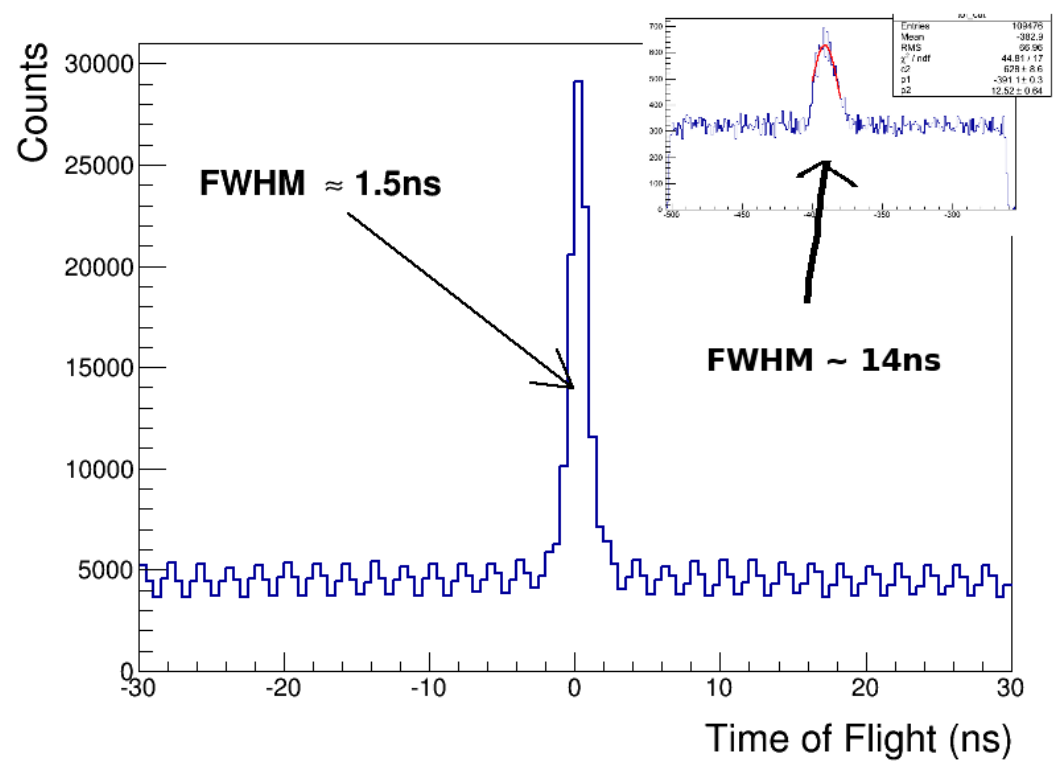

Figure 4.11: Coincidence time comparison before (top right) and after calibrations. Note the RF beam structure visible after calibration.

\subsection{Calorimeters}

Particle identification is primarily achieved through the time of flight and missing mass cuts for these experiments. This is due to constrained kinematics and relatively low beam energies. None the less, there is some contamination that shower calorimeters can detect and correct remove from the data stream. The calorimeters measure energy deposited in lead glass blocks. Each block has a PMT with slightly different response characteristics. We can normalize the PMTs for regular responses by first subtracting the pedestal value (basically the zero point of the PMT), and applying a scale factor to match the peak of the response to a specified value. Figure 4.12 shows the raw PMT readouts, and Figures 4.13, 4.14, 4.15, and 4.16 show the alignment between the channels in each of the four layers of particle identification calorimeters. The layers in the LHRS are the pion rejection layers (PRL1 and PRL2), while the preshower (PS) and shower (SH) calorimeters are in the RHRS. Note that the second layer in each HRS (the shower and PRL2) are not ultimately used in the analysis due to the misfiring of PMTs (SH PMT 41 and PRL2 PMT 14) causing inefficiencies. 
PMT \# vs. L.prl2.a[\#]

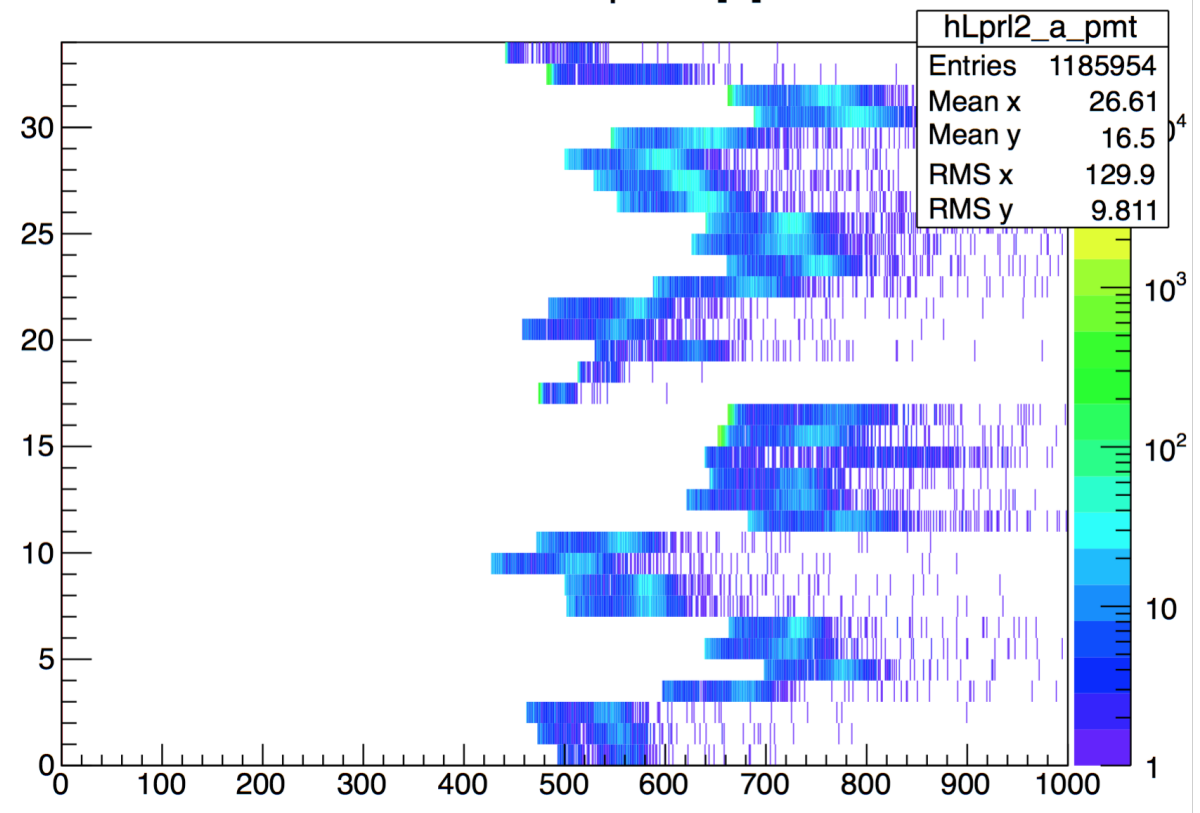

Figure 4.12: PMT number vs PMT response before calibration for pion rejection layer 2 in the LHRS.

PMT \# vs. L.prl1.a_c[\#]

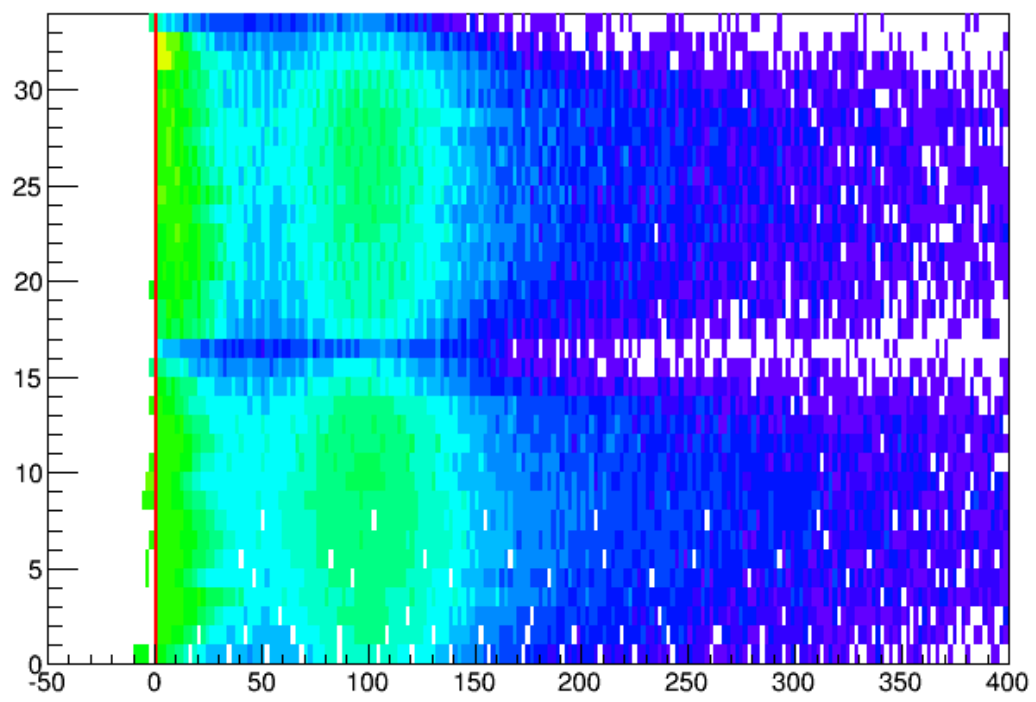

Figure 4.13: PMT number vs calibrated PMT response for pion rejection layer 1 in the LHRS. 


\section{PMT \# vs. L.prl2.a_c[\#]}

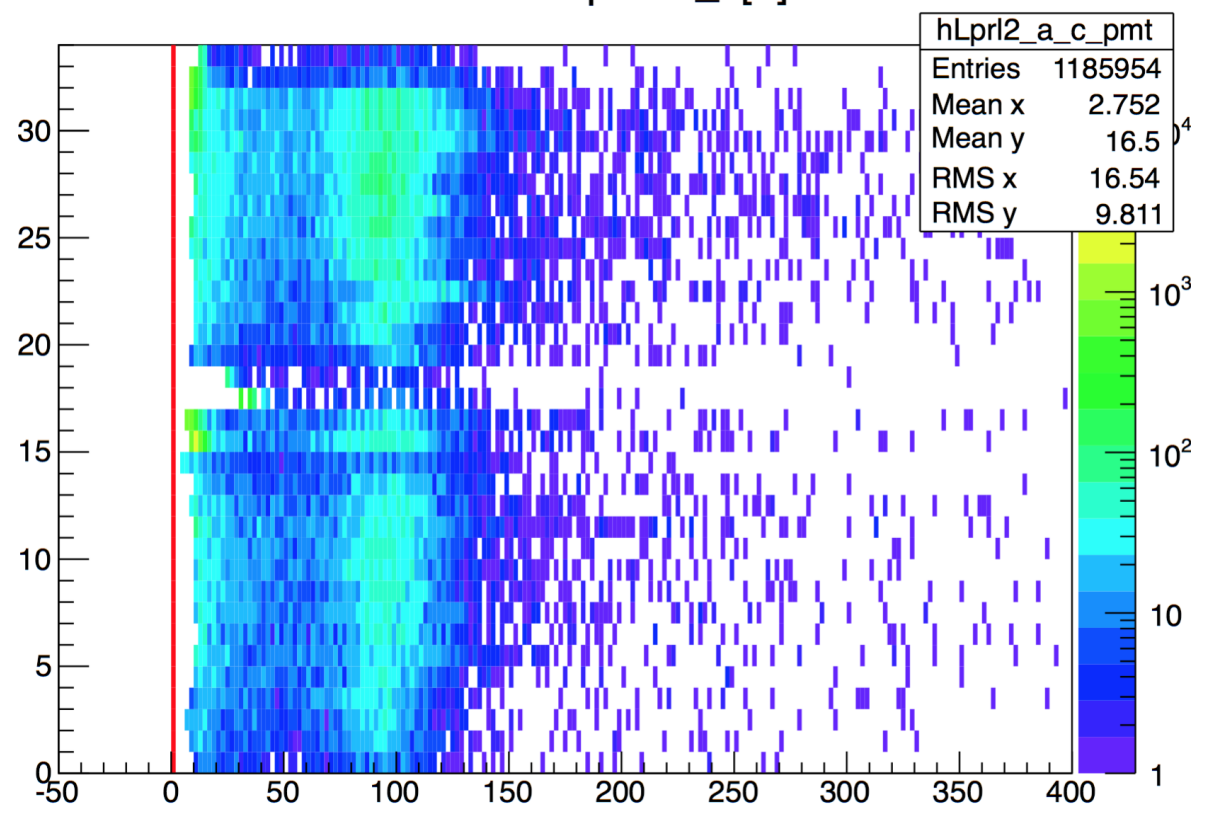

Figure 4.14: PMT number vs calibrated PMT response for pion rejection layer 2 in the LHRS.

PMT \# vs. R.ps.a_c[\#]

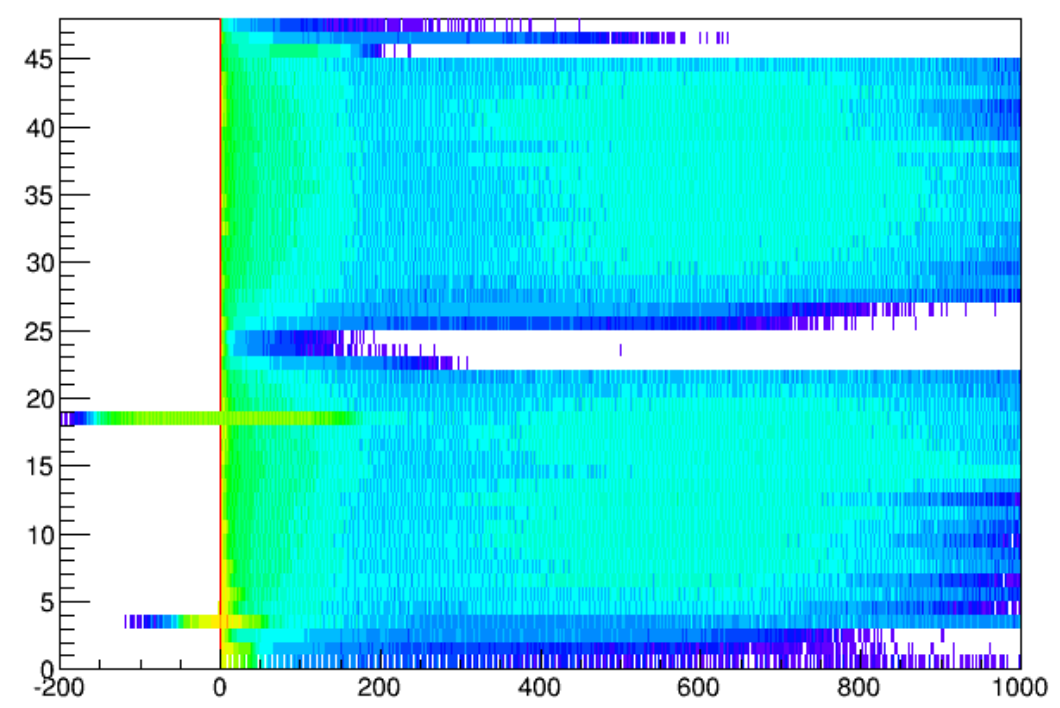

Figure 4.15: PMT number vs calibrated PMT response for the preshower calorimeter in the RHRS. 


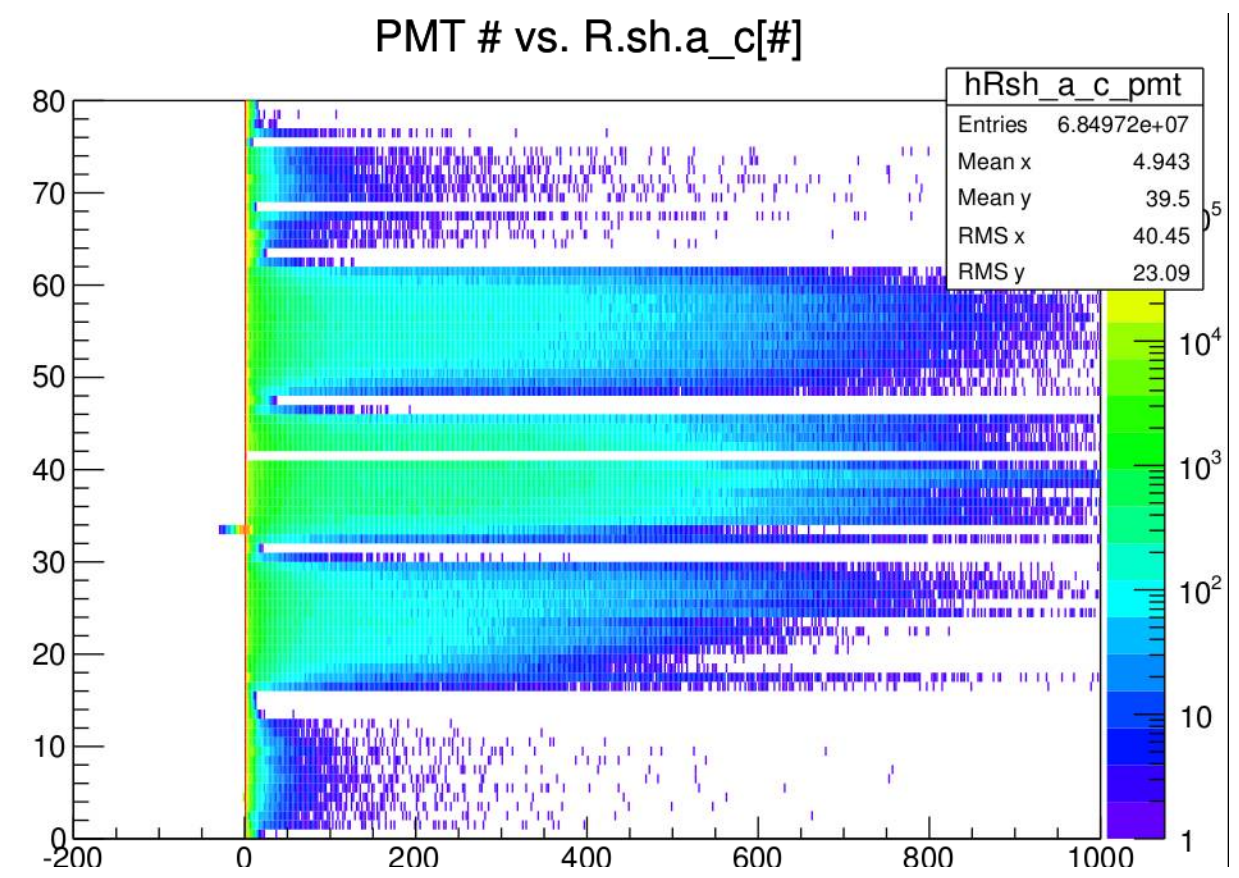

Figure 4.16: PMT number vs calibrated PMT response for the shower calorimeter in the RHRS.

\subsection{Cherenkov Detectors}

The PMTs of the Cherenkov detectors are calibrated in the same way as the calorimeters. The pedestal value is subtracted, and then the spectrum is scaled to align the main signal peak of every PMT. Only the electron spectrometers have Cherenkov detectors installed. Figure 4.17 shows the alignment for JLab RHRS. Of note is the large number of channels with significant events to the right of the zero value.

Figure 4.18 highlights the issue more clearly. The spectra on the right have pedestals that are normal, while those on the left have features and extra width. These structures lead to inefficiencies in the cuts, and so this detector is not used. 
PMT \# vs. R.cer.a_c[\#]

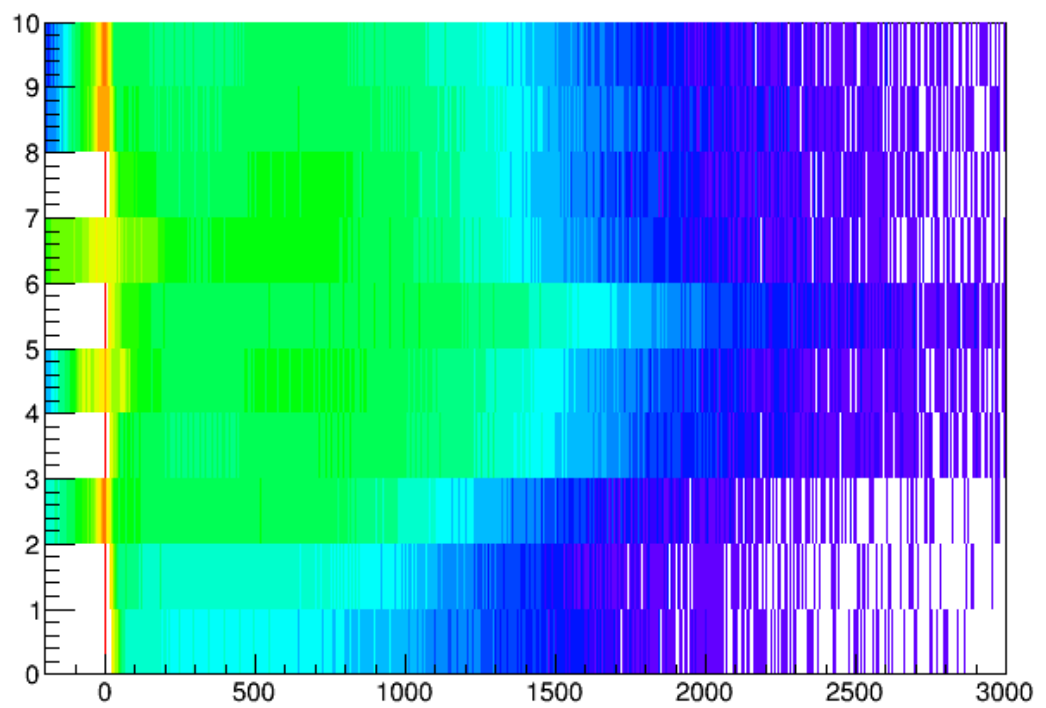

Figure 4.17: Response of the individual Cherenkov PMTs in arbitrary units. Note the wide pedestals in channels half the channels, including the central acceptance. 

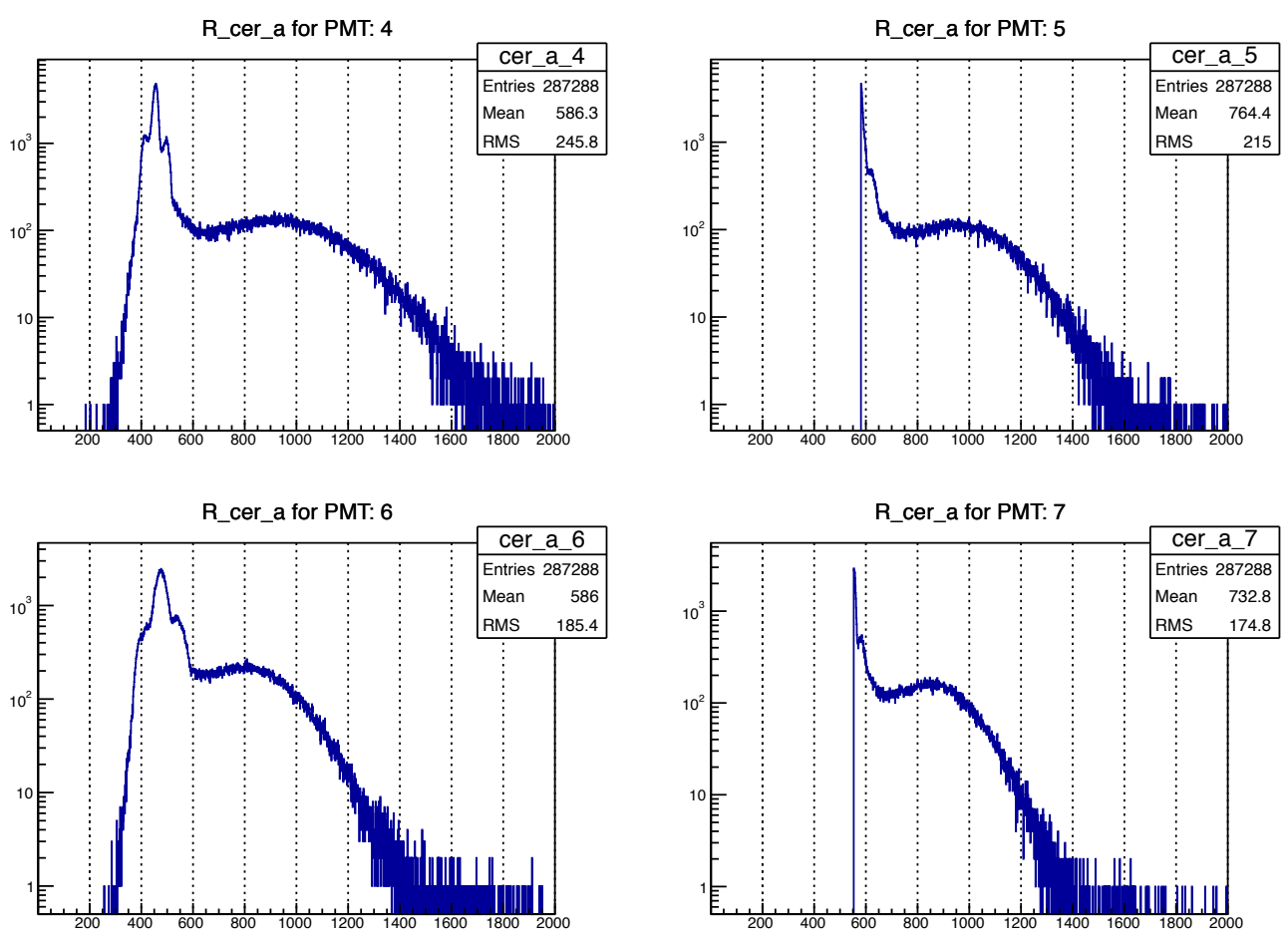

Figure 4.18: Expanded view of the Cherenkov pedestal issues. The left column shows problematic PMT pedestals with multipole peaks. The right column pedestals are much narrower and rise more steeply. 


\subsection{Scintillating Panels}

The scintillating panels responsible for the timing information also record energy deposition, which can be used for particle identification. The calibration process is identical to the Cherenkov detectors and JLab calorimeters: subtract the pedestal position, then scale to align the peak of interest for all channels. 


\section{CHAPTER 5}

\section{DATA ANALYSIS}

\subsection{Background subtraction}

Background signals from random accidentals and spurious tracks from unrelated processes were eleminated from the data set via time of flight background subtraction, and lead glass calorieter particle identification cuts. The background subtraction is accomplished by sampling a wide region of the flat background shown in Figure 5.1, scaling those counts to match the width of the peak cut, and subtracting from the counts passing the peak cut. The wave structure seen in the flat background region of the JLab data is the 2ns RF structure of the electron beam delivered to the experimental hall [65]. A total peak width cut of $10 \mathrm{~ns}$ (20 channels in Figure 5.1) was used for all kinematics at JLab at 6 ns at MAMI. This was chosen as a conservative cut on the timing peak that would line up with the RF background peaks. This cut is not good enough to discern electrons from pions ( $\Delta T \approx 1 \mathrm{~ns}$ ), but will easily differentiate protons and pions ( $\Delta T \approx 50 \mathrm{~ns}$ at JLab, $25 \mathrm{~ns}$ at MAMI). These time of flight calculations are based on $v=\sqrt{|\vec{p}|^{2} /\left(m^{2} c^{2}+|\vec{p}|^{2}\right)}$ with $\mathrm{m}$ being the particle mass, and $\vec{p}$ the spectrometer momentum setting $(\approx 700 \mathrm{MeV})$. The JLab HRS has a drift distance of roughly $20 \mathrm{~m}$, while the A1 spectrometers drift distance is roughly $11 \mathrm{~m}$. Hence, the particle identification power of the timing cut is much stronger for the proton arm, and other detectors must be used in the electron arm.

A region of flat background plateau is chosen on either side of the peak (total width $\approx$ $48 \mathrm{~ns}$ at JLab, and $30 \mathrm{~ns}$ at MAMI), and counts in the two regions are summed, then scaled to match the width of the central peak. This scaled background value is subtracted from the value passing the peak cut to find the counts under the peak, but above the background. 


\section{coincidence time of flight w/o PID cut}

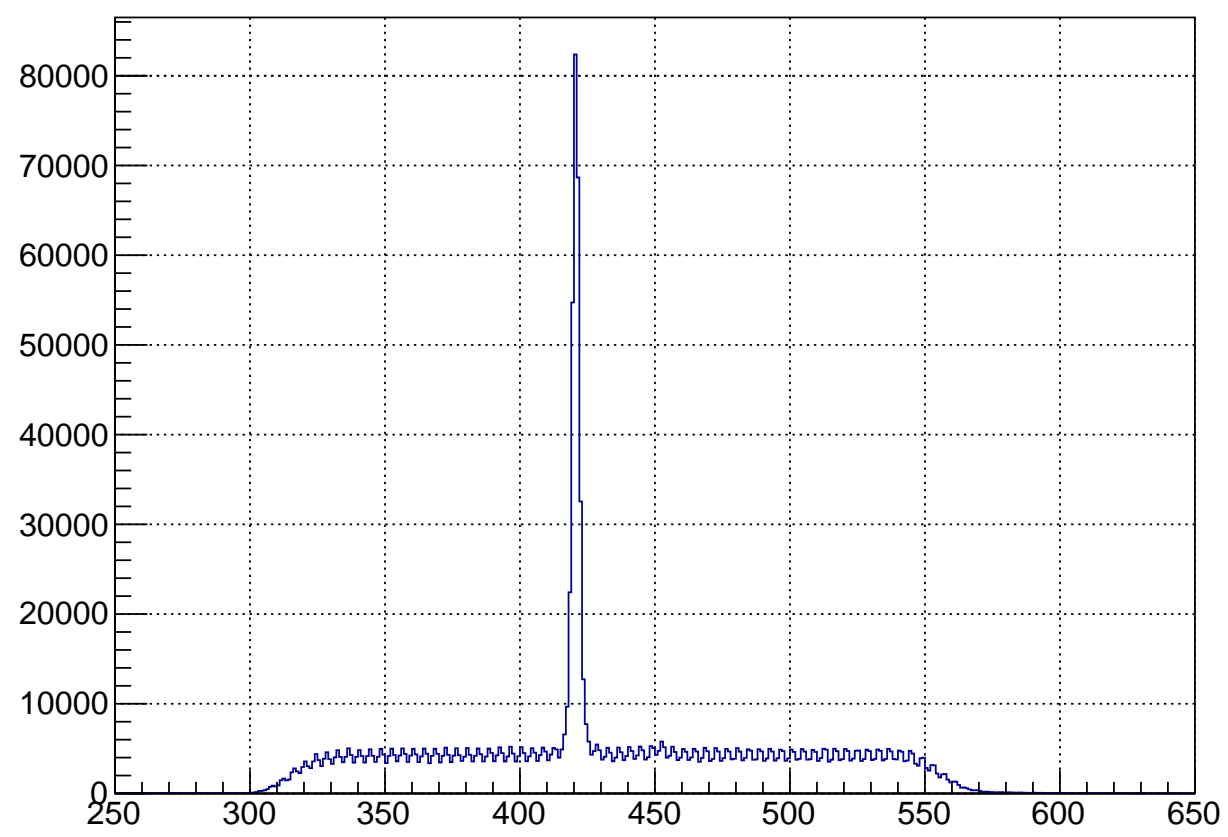

Figure 5.1: Coincidence time of flight for detected particles at JLab in PMT channels ( 2 channels $=1 \mathrm{~ns}$ ). The full width at half maximum of the peak is approximately $1.5 \mathrm{~ns}$, and the $2 \mathrm{~ns}$ RF structure of the background is clearly visible.

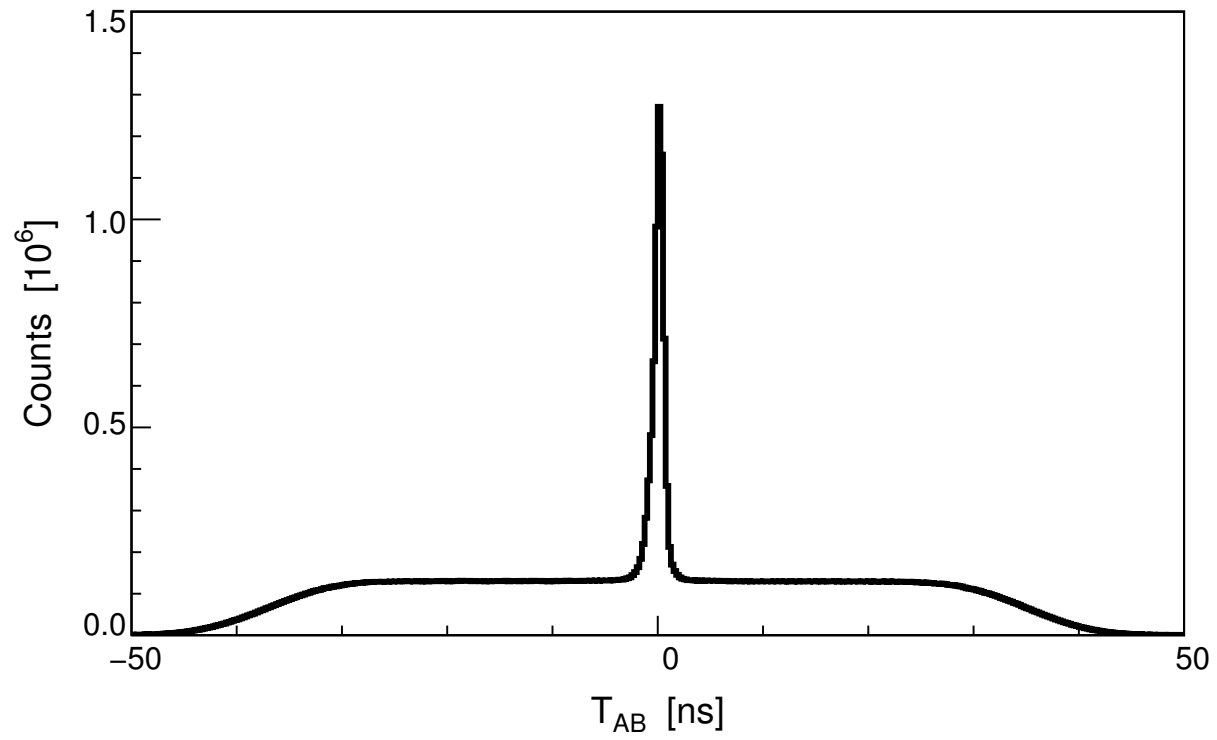

Figure 5.2: Coincidence time of flight for detected particles at MAMI. The full width at half maximum of the peak is approximately $1.1 \mathrm{~ns}$. 
Particle identification using the lead glass calorimeters and Cherenkov detectors was done in three steps. First the PMTs were calibrated by normalizing the peak of the spectrum of $E / p$ (energy deposited devided by particle momentum) for each channel of the detector. This was done with very strict cuts on the spectrometer acceptance and kinematic variables in order to minimize the number of uncorrelated background events.

Second, the response of the detectors was characterized to ensure the intended particles are being cut. Figure 5.3 shows the spectrum from the electron preshower comparing the full spectrum to a scaled spectrum where electrons are selected in the Cherenkov detector (only a single Cherenkov channel spectrum is used for this check due to pedestal issues). The first peak in the full spectrum is clearly cut by the Cherenkov selection. Figure 5.4 shows the same process inverted to highlight the Cherenkov spectrum. The histogram of the preshower selected events shows an electron signal that is attenuated by a factor of 5 , indicating the $\mathrm{E} / \mathrm{p}$ cut selects pions preferentially, but that an efficiency factor will be required to account for misidentified events.

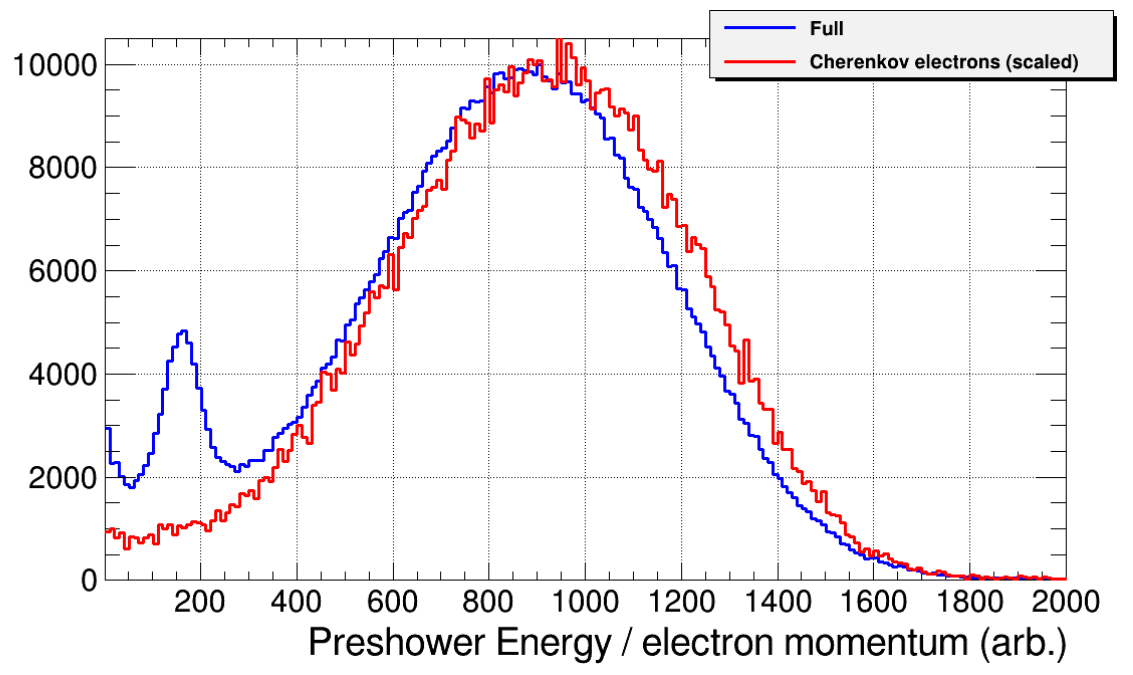

Figure 5.3: The electron arm preshower detector over electron momentum cut spectrum is shown with and without electrons being selected by the Cherenkov detector.

Finally, an estimate the cut efficiency is made to account for the true events lost in the cut. This estimate is performed either by analyzing the PMT spectrum and fitting the data peak to integrate the events lost as in Figure 5.5, or by analyzing the time of flight plot to detect the change in events under the timing peak as in Figure 5.7. These methods were compared for consistency. 


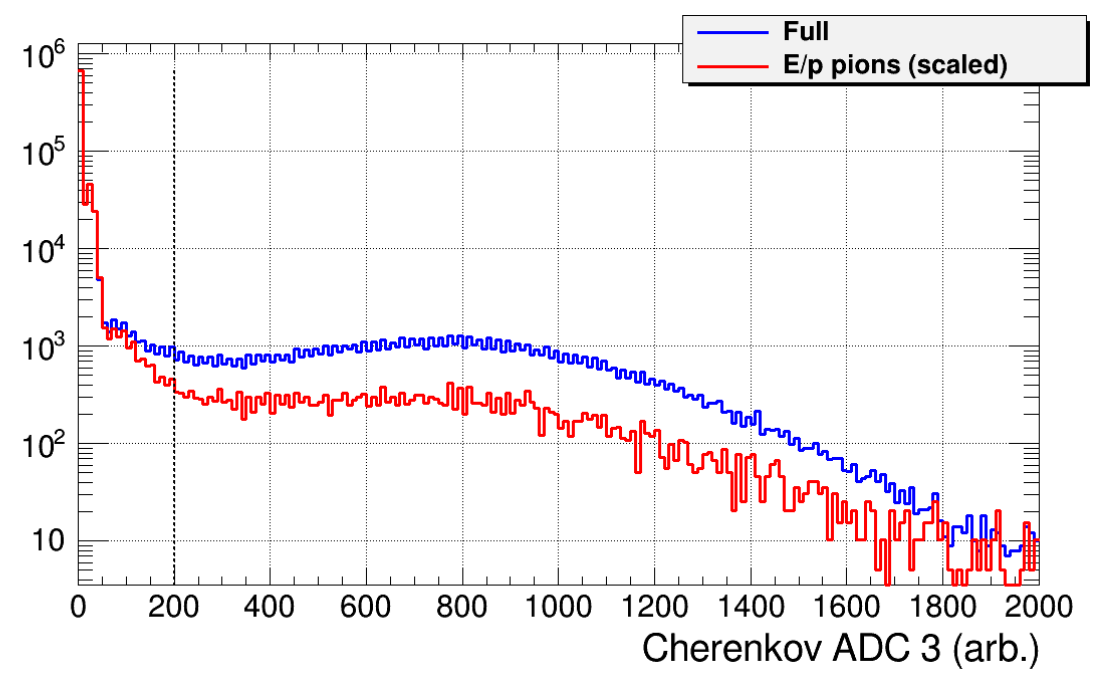

Figure 5.4: The electron arm cherenkov detector spectrum is shown with and without electrons being selected by the preshower detector.

shower E over $p$, run:2524

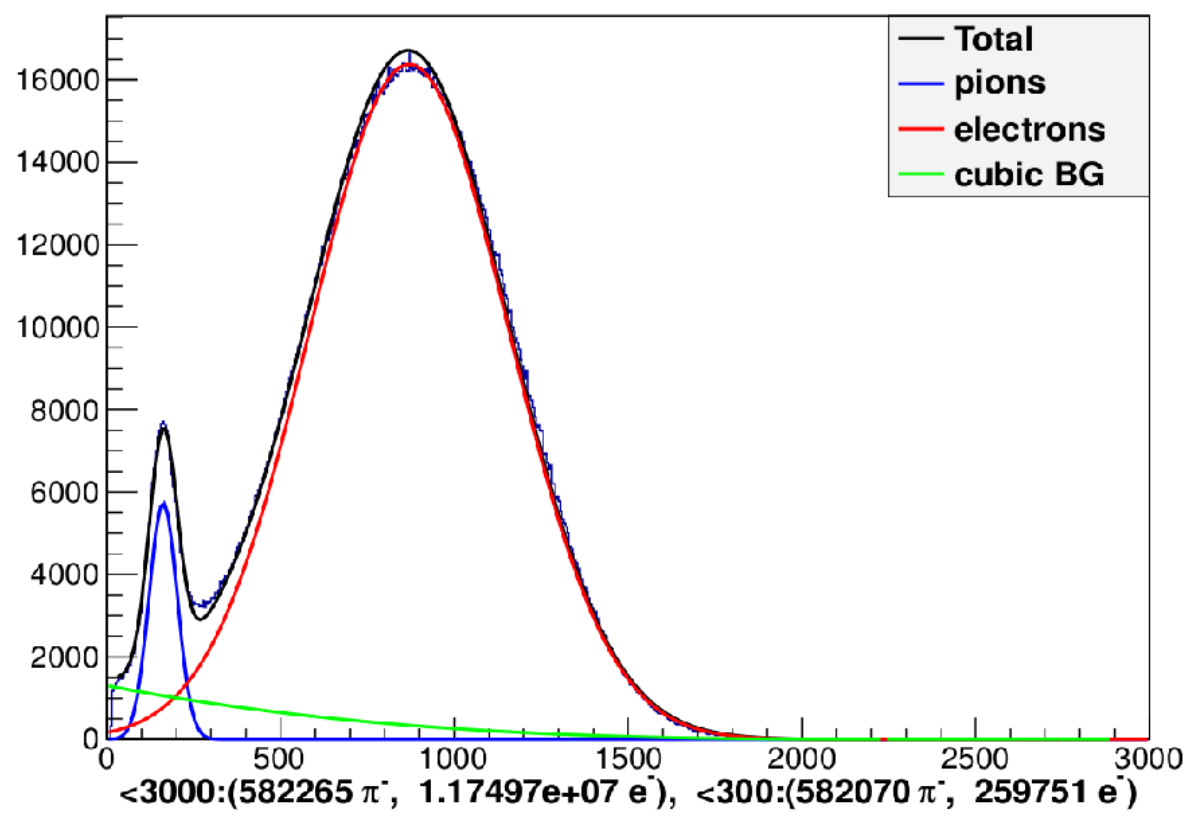

Figure 5.5: Lead glass calorimeter particle identification process for electron arm at JLab. The indicated region was cut from the data. 


\section{PRL1 E / P, no TOF cut}

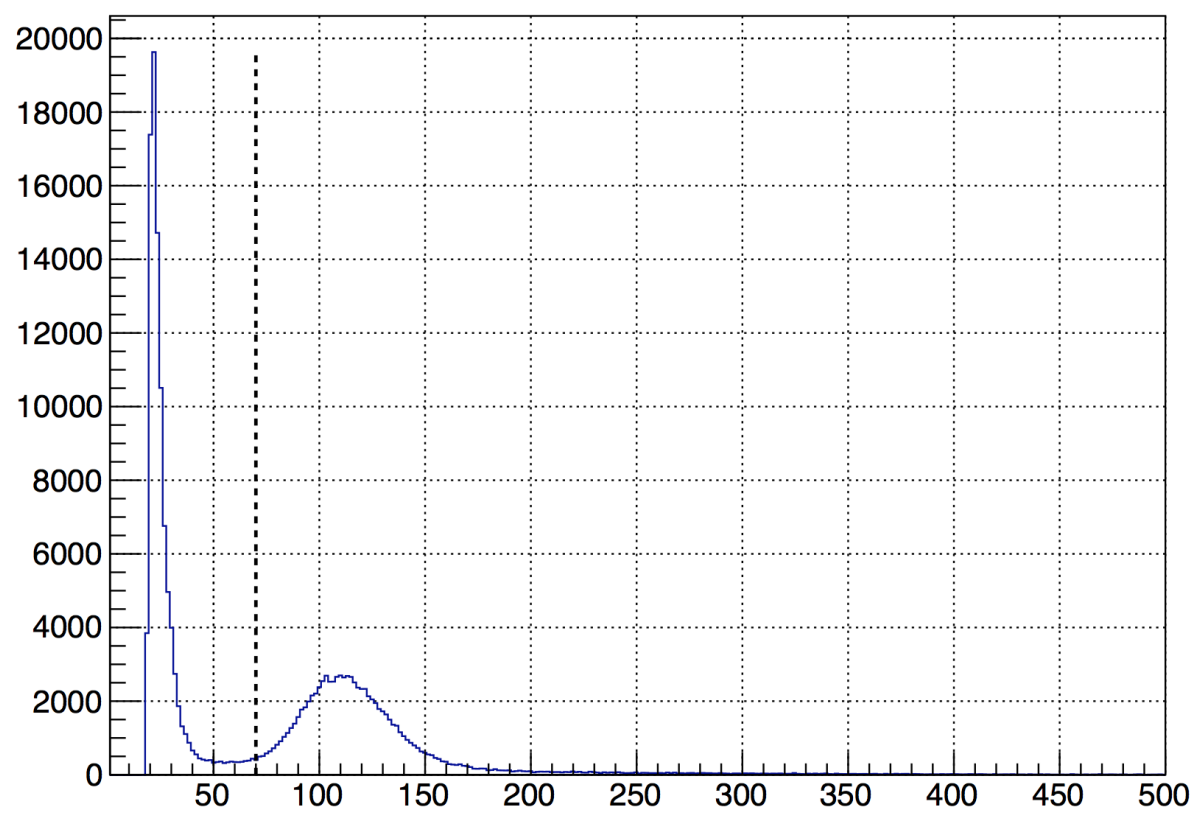

Figure 5.6: Lead glass calorimeter particle identification process for proton arm at JLab. Events below indicated line are cut from the data.

An additional background subtraction step was required for the MAMI data. The VCS channel competes with the pion electroproduction channel with a branching ratio 100 times less likely. Despite good separation in missing mass, there is still some overlap between the two signals which must be accounted for. Thus, for each run the pion tail is fitted and subtracted from the data, this can be seen in Figure 5.8. 


\section{coincidence time of flight $w /$ anti-PRL cut}

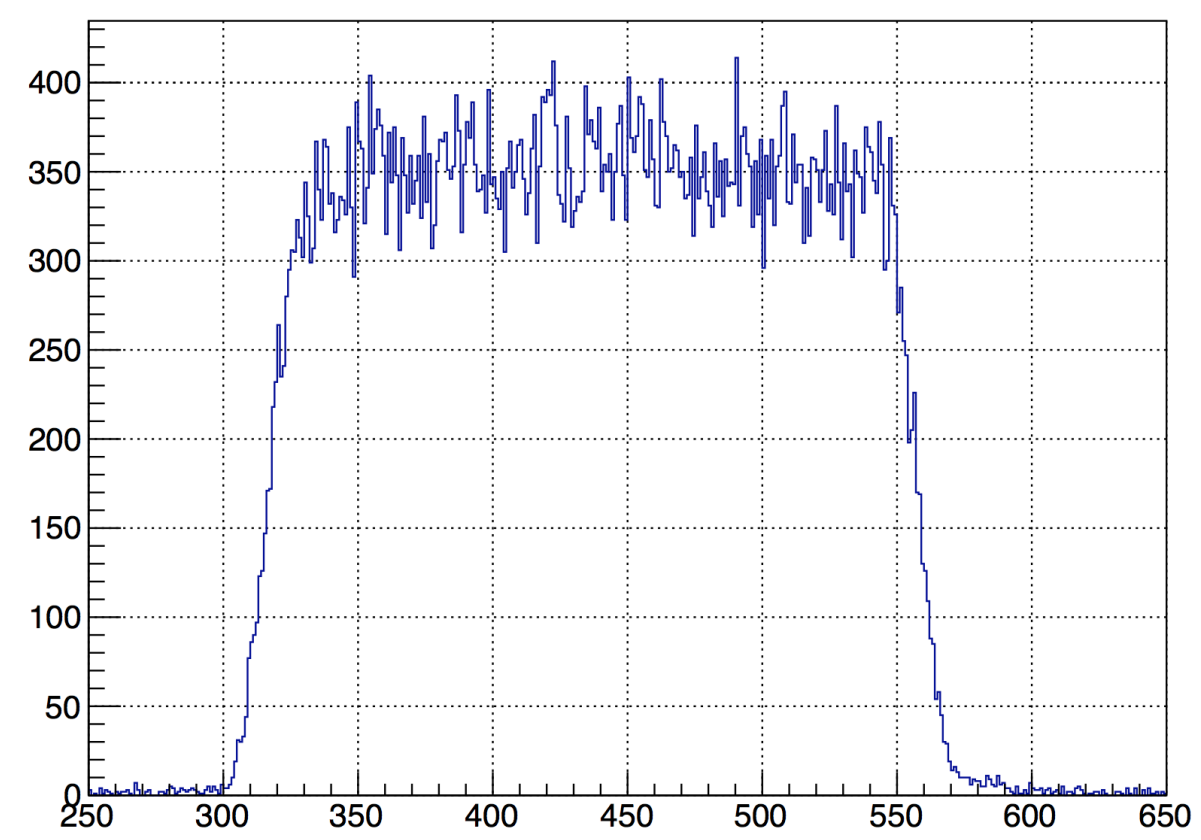

Figure 5.7: Coincidence time plot for events discarded in the cut shown in figure 5.6. Axes are counts per bin vs channels. Lack of a timing peak indicates events eliminated are not from timing peak.

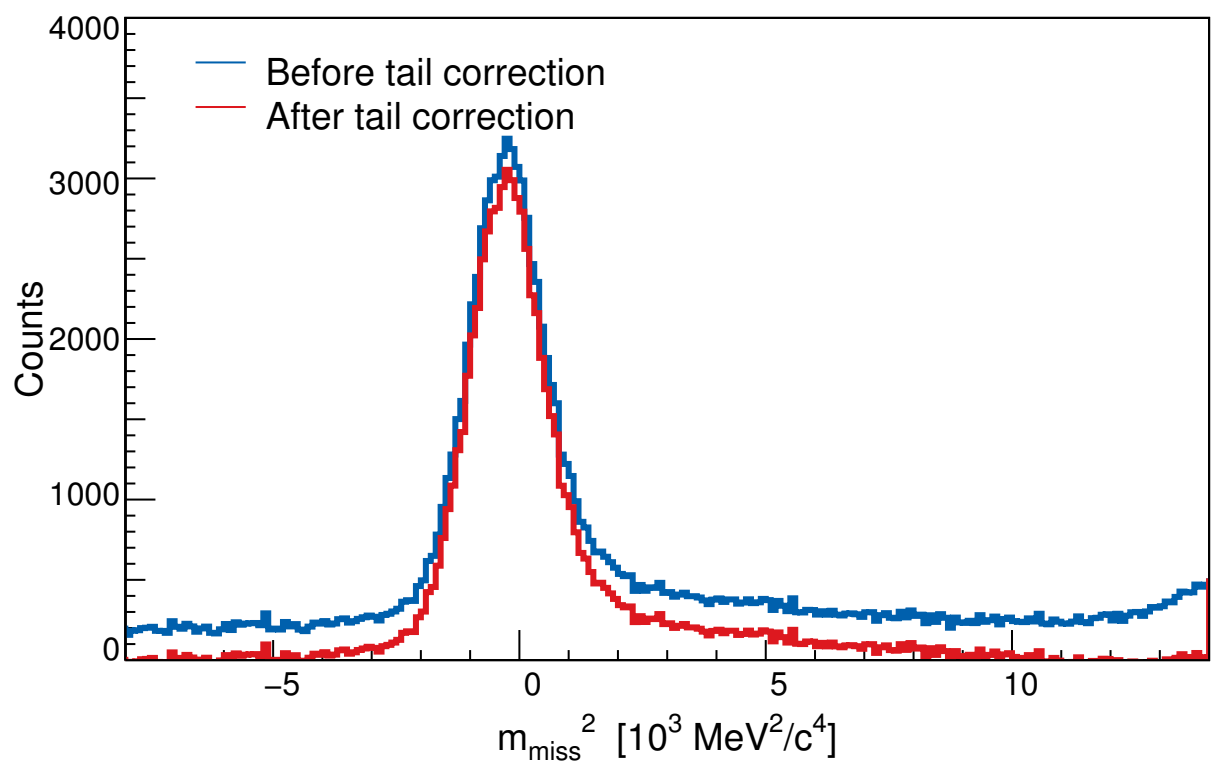

Figure 5.8: Missing mass peak for VCS events in MAMI experiment A1/03-12 before and after subtracting the pion tail. 


\subsection{Spectrometer Momentum}

Early in the analysis, it was shown that the missing mass spectrum at JLab was not aligning to the theoretical value. It was suspected this was due to the calibration of the HRS spectrometer magnets. An analysis was conducted to check the momentum setting of each arm to recalibrate the central momentum setting for every kinematic setting.

For each asymmetry pair kinematic, there are two sets of kinematics where the momentum settings of the spectrometers don't change, but their positions do. This allows a calibration process where the momentum setting for one arm (e.g. the electorn arm) is presupposed to be a certain value, and the other is optimized to center the missing mass spectrum at the theoretical value. This yields two lines with slightly different slopes, and their intersection gives the calibrated values for the two spectrometer momentum settings. Figure 5.9 shows this process.

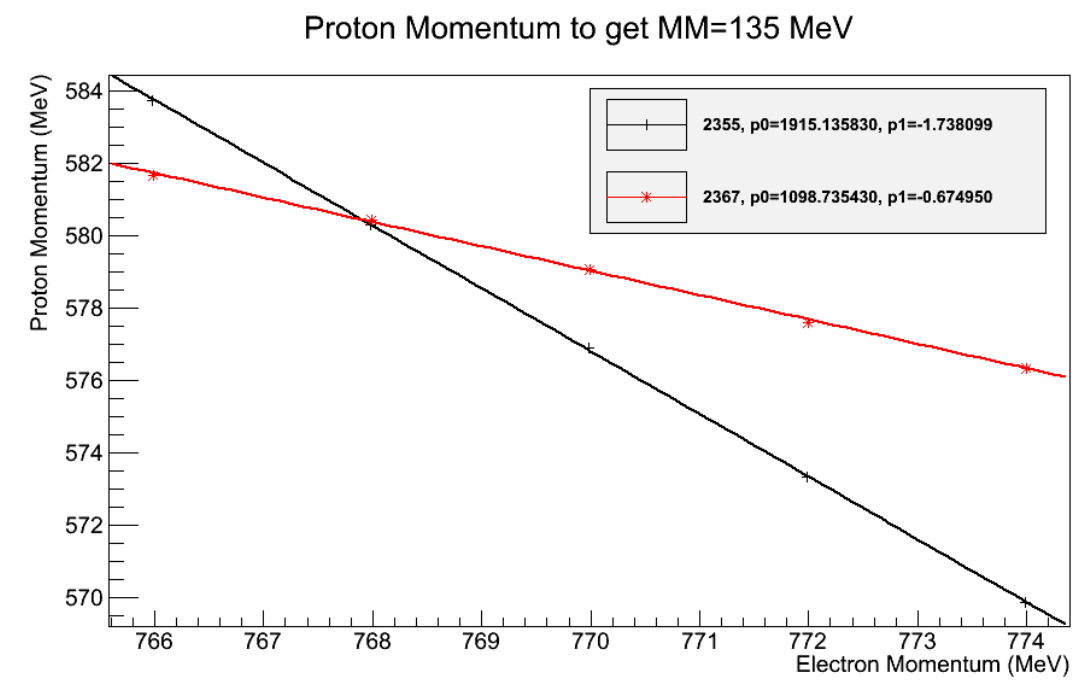

Figure 5.9: Analysis of spectrometer magnet momentum settings. Each line represents one setting for an asymmetry pair where the spectrometers are moved, but the magnet settings are not changed. For each point, the electron momentum is fixed, and the proton momentum setting tuned to match the missing mass peak at the theoretical value. The intersection of the lines gives the optimized momentum settings.

Coupled with this analysis at JLab is an energy loss correction. As the charged particles move from the reaction vertex to the detector plane, they encounter many materials, and may lose energy while passing through each. A portion of the analysis package is devoted to tracking the path length through each material based on the tracking information, and 
applying Bethe-Bloch stopping power and the Density Effect Corrections as in [74] for each material. The particle momenta are corrected for the energy lost in each material, and the missing mass is recalculated. The effect, coupled with the calibration analysis above result much better alignment of the missing mass peak throughout the experiment. Figure 5.10 shows how the JLab missing mass spectrum comes into alignment with theory after the momentum optimizations.

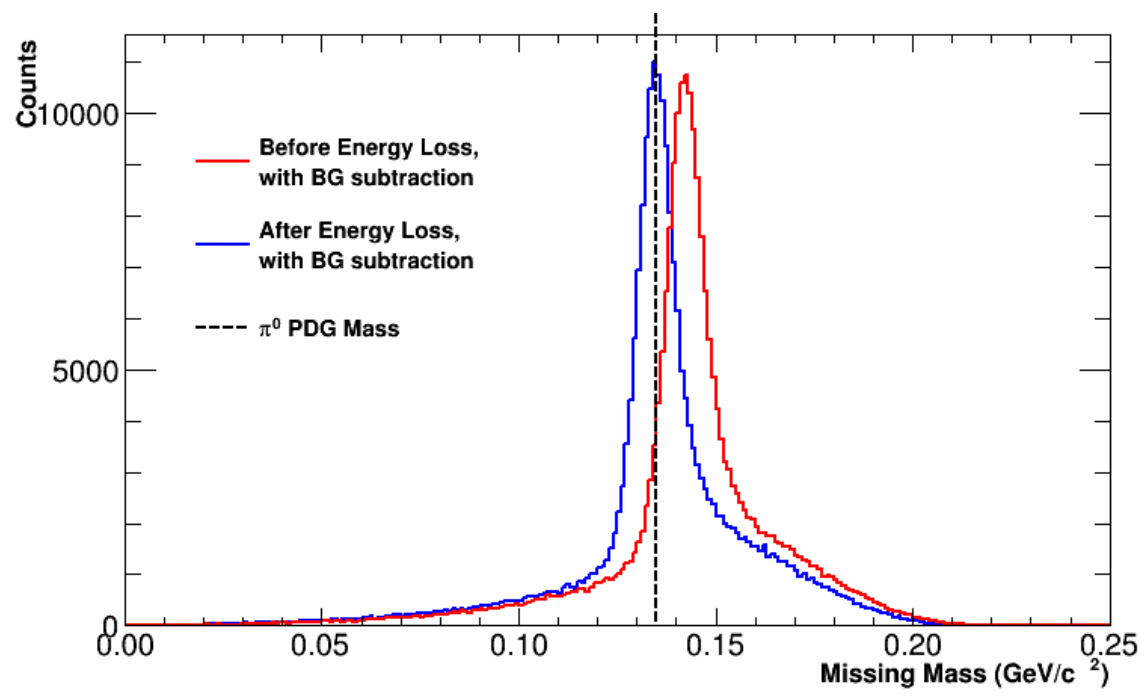

Figure 5.10: Corrections to the missing mass peak from spectrometer momentum optimization and energy loss corrections provide much better agreement in the missing mass spectrum with theory.

A similar method was used at MAMI for control fluctuations in the missing mass spectrum. MAMI sometimes has issues with frost accumulating on the target walls, slightly altering the momentum of the detected particles. This is shown in the data as instabilities in the missing mass, see Figure 5.11. Software is in place to account for these affects (called snow corrections in other works), but I was unable to find a stable solution despite much effort. This was resolved by using the method above to optimize the spectrometer settings. Pairs of runs were selected from associated kinematics settings were chosen to run together. The analysis was repeated with three associated runs to ensure consistent results. The final momentum settings are shown in Figures 5.12 and 5.13. Note the tight scale in the $y$ axis, indicating the corrections are small in magnitude and stable. That the final set points are less than $0.5 \%$ from design in the proton arm (which had issues with magnet NMR probe), and $0.1 \%$ for the electron arm. The initial and final missing mass peak evolution for the MAMI data is shown in Figure 5.14. 


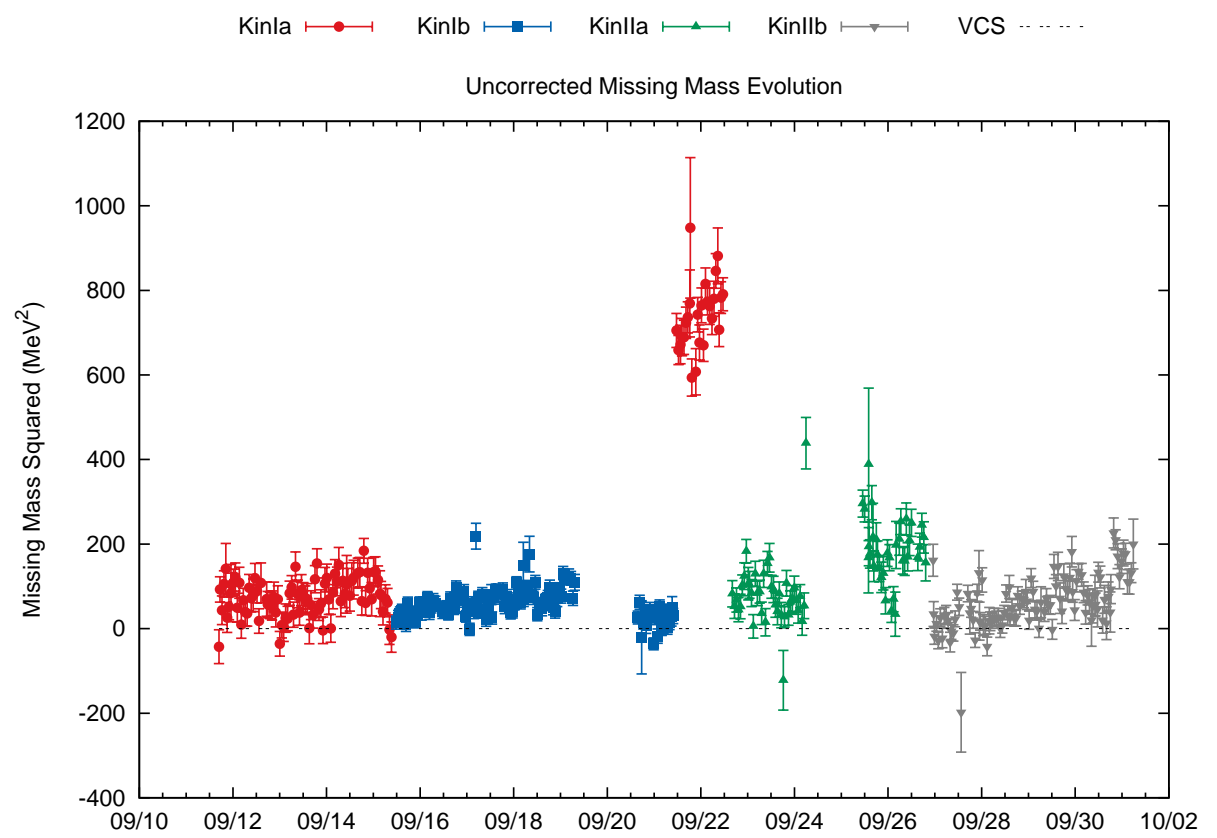

Figure 5.11: Instability in the VCS missing mass peak during the MAMI beamtime.

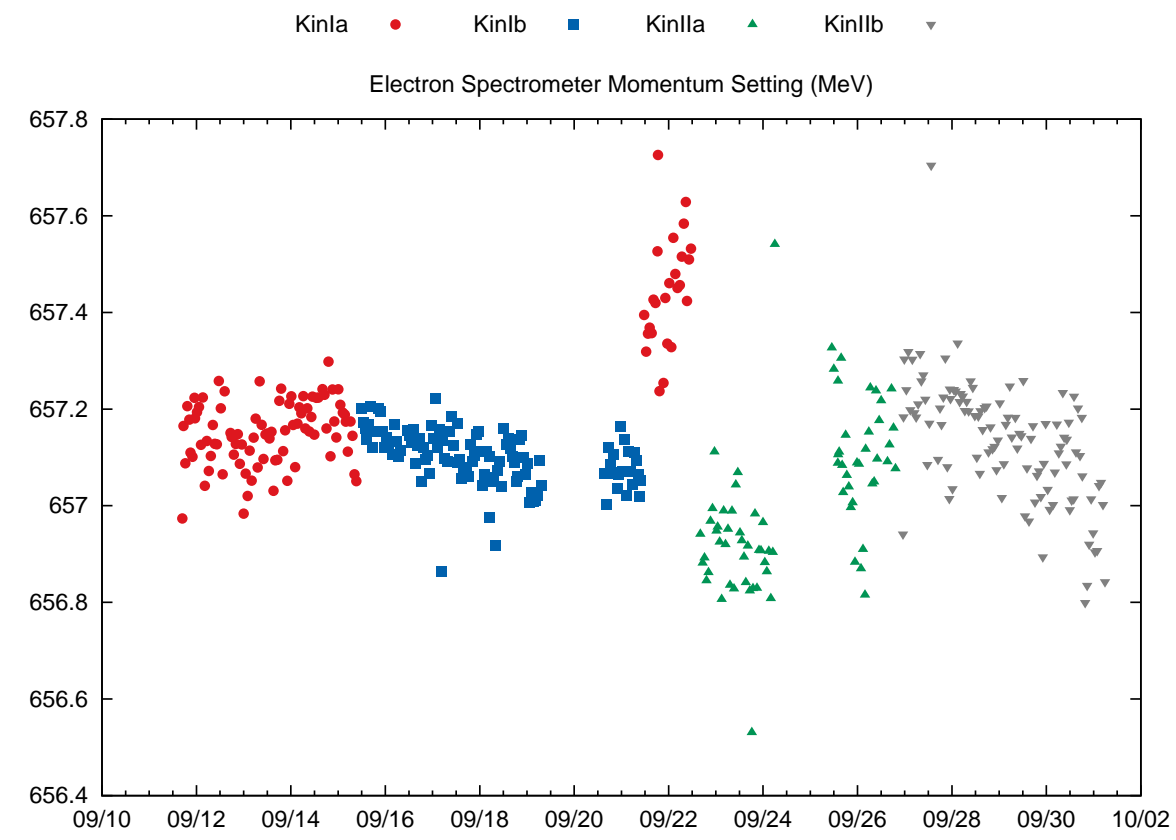

Figure 5.12: Optimized electron arm momenta for MAMI beamtime. Note the total vertical scale represents $0.1 \%$ fluctuation from the center. 

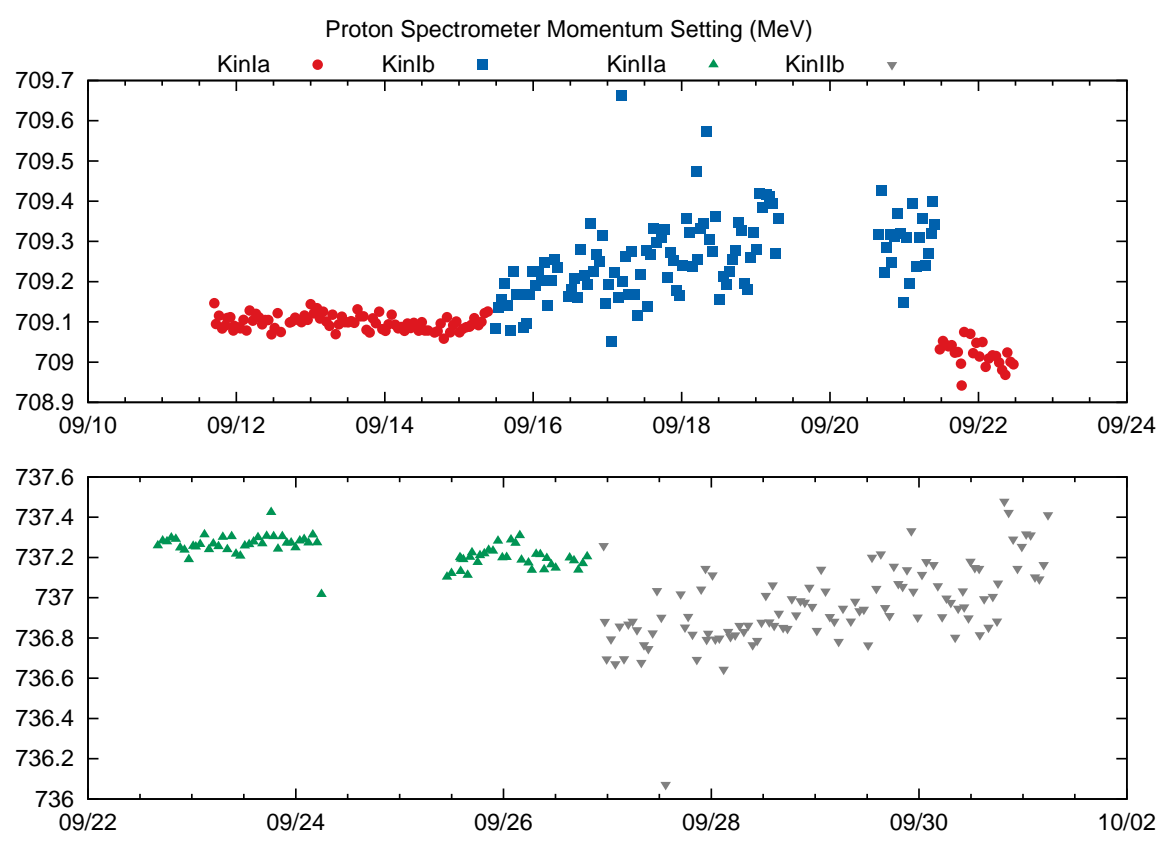

Figure 5.13: Optimized proton arm momenta for MAMI beamtime. Note the total vertical scale represents less than $0.1 \%$ fluctuation from the center.
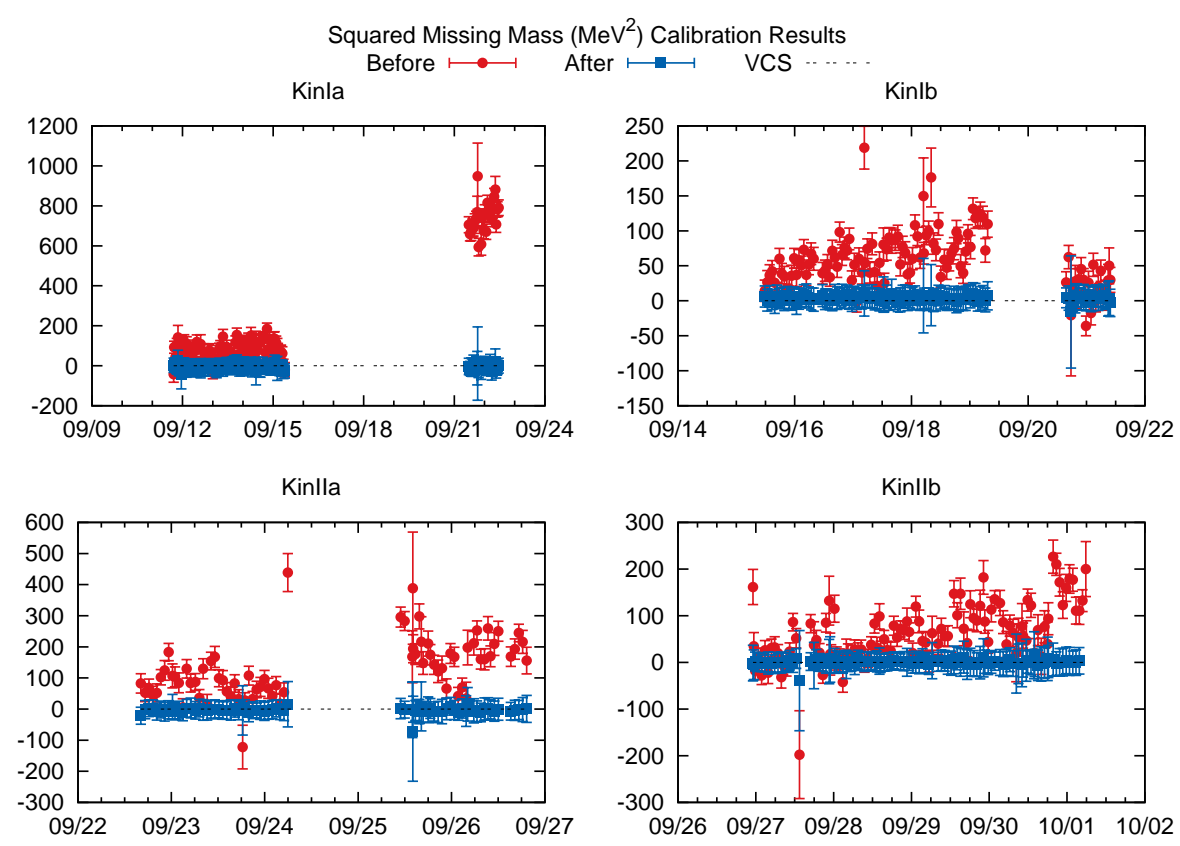

Figure 5.14: Comparison of initial and optimized missing mass evolution during the MAMI experiment. 


\subsection{Luminosity}

The luminosity is calculated from accumulated charge, target density, and target length. The accumulated charge was calculated from the experiment between each scalar readout in the JLab data stream, approximately every ten seconds. The current, as measured by calibrated beam current monitors, is multiplied by the running time since the last readout, and added to a running total. This method accounts for small fluctuations in beam current during stable segments of operation, as well as for the loss of current during beam drops.

The target length at JLab is nominally known, but adjusted for the length contraction for the cold temperature of the experiment, and the curvature of the endcaps in the region the beam was monitored to be hitting the target, as seen in Figure 5.15.

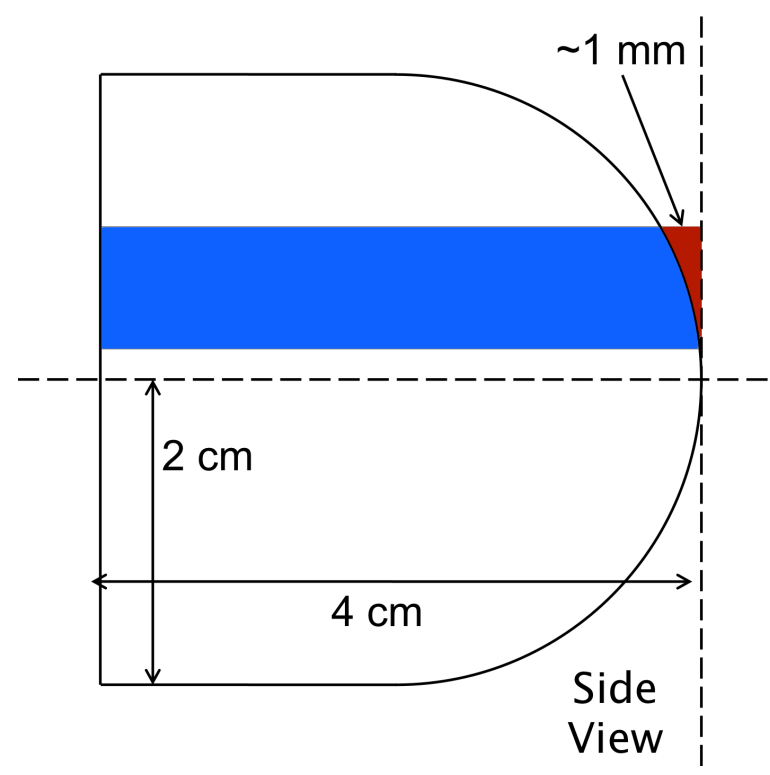

Figure 5.15: JLab target length analysis.

Target density was adjusted based on the beam current for the two JLab targets. The high energy electron beam causes a local change in density where the beam is interacting. To model this change, the reaction rate, $\mathrm{R}$, was supposed to be $R=c I+R_{0}$ with $\mathrm{I}$ being the beam current, $c$ being a constant factor depending on the target materials, liquid hydrogen and aluminum, and $R_{0}$ being the reaction rate when current is zero. The factors were caclulated by considering data taking with no current (cosmics runs), data taken with beam on a dummy target containing no hydrogen, and data from the experimental cryotarget cells with varying beam current. The target density correction is written as $\rho=\rho_{0}(a I+1)$, 
where $\rho$ is the effective target density used in the luminosity calculation, $\rho_{0}$ is the nominal target density of $0.0723 \mathrm{~g} / \mathrm{cm}^{3}$, and a is the current dependent density correction factor. These factors were generally a few percent adding a systematic uncertainty less than $0.5 \%$ to the final result. See [58] for more details.

Table 5.1: Luminosity factors at JLab. Density Correction is a correction to $0.0723 \mathrm{~g} / \mathrm{cc}$. Uncertainties are given as a percentage of the value.

\begin{tabular}{|l|c|c|c|c|}
\hline Kin & $\begin{array}{c}\text { Dens. Corr. } \\
(\%)\end{array}$ & $\begin{array}{c}\text { Beam Time } \\
(\mathrm{min} \pm \%)\end{array}$ & $\begin{array}{c}\text { Cum. Charge } \\
(\mathrm{C} \pm \%)\end{array}$ & $\begin{array}{c}\text { Targ. Len. } \\
(\mathrm{cm} \pm \%)\end{array}$ \\
\hline 1 & 0.65 & $640.50 \pm 0.0163$ & $0.5760 \pm 0.00298$ & $3.86 \pm 0.104$ \\
\hline 2 & 0.65 & $982.09 \pm 0.0129$ & $0.8719 \pm 0.00224$ & $3.86 \pm 0.104$ \\
\hline 3 & 0.87 & $976.11 \pm 0.0129$ & $1.4044 \pm 0.00161$ & $3.86 \pm 0.104$ \\
\hline 5 & 3.49 & $135.87 \pm 0.0346$ & $0.6143 \pm 0.00225$ & $3.86 \pm 0.104$ \\
\hline 6 & 1.74 & $559.93 \pm 0.0170$ & $1.3178 \pm 0.00147$ & $3.86 \pm 0.104$ \\
\hline 7 & 3.49 & $393.63 \pm 0.0203$ & $1.8142 \pm 0.00149$ & $3.86 \pm 0.104$ \\
\hline $8 \mathrm{~S}$ & 3.49 & $148.65 \pm 0.0331$ & $0.7067 \pm 0.00266$ & $3.86 \pm 0.104$ \\
\hline 11 & 2.40 & $430.06 \pm 0.0195$ & $1.3829 \pm 0.00178$ & $3.86 \pm 0.104$ \\
\hline 12 & 3.49 & $296.36 \pm 0.0242$ & $1.3310 \pm 0.00178$ & $3.86 \pm 0.104$ \\
\hline $8 \mathrm{~L}$ & 4.79 & $94.659 \pm 0.0415$ & $0.1855 \pm 0.00372$ & $14.8 \pm 0.135$ \\
\hline 9 & 3.60 & $338.68 \pm 0.0219$ & $0.6045 \pm 0.00210$ & $14.8 \pm 0.135$ \\
\hline 10 & 5.99 & $237.13 \pm 0.0262$ & $0.6750 \pm 0.00204$ & $14.8 \pm 0.135$ \\
\hline 13 & 4.19 & $133.69 \pm 0.0349$ & $0.2772 \pm 0.00315$ & $14.8 \pm 0.135$ \\
\hline 14 & 4.19 & $103.14 \pm 0.0397$ & $0.2131 \pm 0.00354$ & $14.8 \pm 0.135$ \\
\hline
\end{tabular}

The target cell at MAMI A1 is much smaller than the cells at JLab. The length is comparable to the short target used at JLab, but the end caps are much more square, and the target diameter is roughly $25 \%$ that of the JLab targets. Additionally, the typical beam current during the MAMI run time was much less than those at JLab, just $15 \mu \mathrm{A}$ compared to the nearly $80 \mu \mathrm{A}$ at JLab. These factors mean the boiling effects seen at JLab are not present at Mainz.

Related to the low currents at MAMI were issues with the Phillips heating device responsible for cooling the cryotarget system. Inefficiencies in the cooling apparatus lead to large scale instabilities in the target density, as seen in Figure 5.16. The target cell temperature and pressure are checked and stored regularly Aqua++ data stream. These values were used to calculate the target density for each point, and calculate a weighted average for the run, weighted according to the time since the previous recording. The code checked for large $(>0.2 \%)$ fluctuations during the run and either flagged the run, or printed a Target.Density value into the run.db file. 


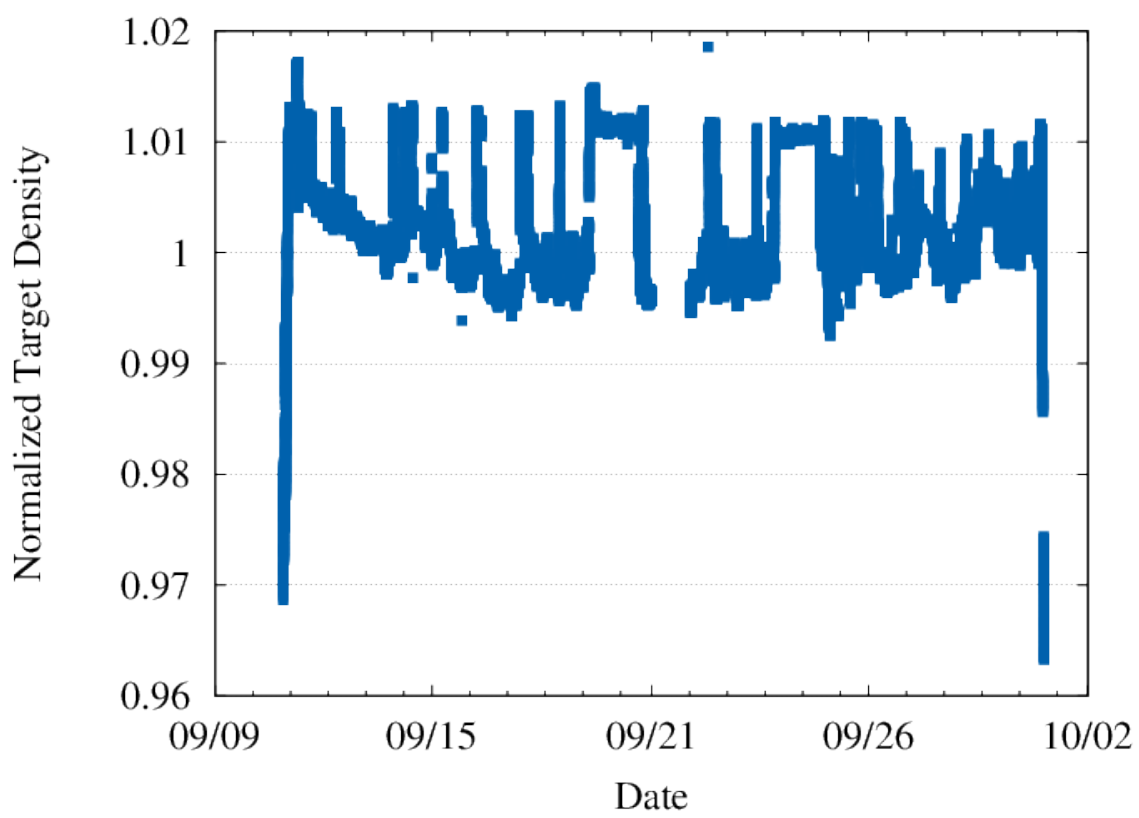

Figure 5.16: MAMI target density during beam time. Tails at either end correspond to target commissioning and decomissioning. Fluctuations per run are less than $0.25 \%$.

\subsection{Proton absorption}

Due to the low momentum settings in the proton spectrometer, there is substantial risk that the proton scattered from the reaction point will be absorbed by the target before exiting. A pathlength is calculated for each material in the proton path from the reaction point to the scintillating plane. The pp and pn absorption cross sections are calculated using a cubic polynomial fit of the data available from the Particle Data Group. Each material is assumed to have an absorption cross section equal to a 50:50 split of protons and neutrons totaling to the material's molecular mass. This gives the probablility of absorption before triggering the detector, and is used as a correction factor to the data. The target cell at MAMI is designed to minimize these effects, using a narrower cell with thinner walls than the JLab targets. Thus, the corrections at MAMI are $0.3 \% 0.5 \%$ for inside and outside kinematics, compared to the $1-2 \%$ correction needed at JLab. 


\subsection{Multiple Track Events}

Multiple tracks recorded in a single event are usually cut out of data analysis to prevent bookkeeping mistakes. Often these events do not amount to a significant portion of the data set, but this was not the case in E08-010. Due to relatively high data rates, as much as 25 percent of the data recorded contains multiple tracks in the N-Delta experiment.

Recovering these tracks can significantly improve the statistical precision of this experiment, but one must proceed cautiously so as not to bias the data. One should also check that the rewards will be meaningful before expending the effort to attempt the multiple track reconstruction.

Once one decides to salvage multiple track events, the process is fairly straightforward. The challenge in the Hall A Analyzer package is to associate the proper timing information with the track position and momentum information for each event. These values are nontrivial to connect because each is measured with a different detector that adds a separate layer of multiplicity to the event. The VDCs provide the position and momentum information for a number of tracks in each event, and the Scintillating planes provide the timing information for a number of hits in each event. Together the tracks and hits determine the total multiplicity for each arm. In E08-010, this information for the two arms must then be combined to determine the final event timing and track reconstruction information for the reaction.

This analysis is only possible due to the work M. Paolone did to add multiple hit PMT TDC output to the analyzer source code. Make sure that the R.s2.nlmrhit variable is available when a block R.s2 . * line is in your analyzer odef file.

Before proceeding, one should get an understanding of the expected results. This is most easily done by quantifying the number of events that pass single or multiple track cuts in each arm. To show the expected results of the multi track study, a table showing the percentage of events passing a number of cuts in each arm was made (See Table 1). This table helped limit the scope of this study to events with a single track in at least one arm. The top left cell (highlighted green) is the only cell included in the single track cut common in other experiments. The first row and column (highlighted yellow) are cells addressed in this document, selected with a single track in either arm cut. The rest of the table (highlighted red) is ignored by this document. This table demonstrates that for the selected kinematic setting, only 72 percent of the data set is addressed when a single cut track is employed. Additionally, up to 25 percent of the data set may be recovered using 
a preliminary multiple track analysis, and only 2 percent of the data is left out after that analysis.

Table 5.2: Percentage of events passing each cut.

\begin{tabular}{|c|c|c|c|c|c|}
\hline \multicolumn{2}{|c|}{$\begin{array}{c}\text { Number of } \\
\text { tracks in }\end{array}$} & \multicolumn{4}{|c|}{ L arm } \\
\cline { 2 - 6 } & 1 & 2 & 3 & $>3$ \\
\hline \multirow{4}{*}{$\mathrm{R}$ arm } & 1 & 72.24 & 7.68 & 1.99 & 0.22 \\
\cline { 2 - 6 } & 2 & 10.54 & 1.11 & 0.28 & 0.03 \\
\cline { 2 - 6 } & 3 & 4.38 & 0.44 & 0.09 & 0.01 \\
\cline { 2 - 6 } & $>3$ & 0.87 & 0.09 & 0.02 & 0.00 \\
\hline
\end{tabular}

It should be stressed again that this analysis should be repeated for every kinematic setting in the experiment. The proportions of events left out may change significantly even if data rates were normalized before data was taken.

Having established that a consequential proportion of the data can be salvaged via multiple track reconstruction, one must then ensure that the events recovered comprise a valid data set. One such way to do this is to compare various spectra. The dominant example is the coincidence timing spectrum. Demanding a sharp timing peak with broad, flat background plateaus ensures the multiple track data is valid. Additionally, one should check that the signal to noise ratio (SNR) of the multiple track data is the same as the single track data. One expects the data to behave in a similar fashion regardless of how many tracks appeared at the detector in a given event. Differences in these plots indicates there may be some other physics differences between the single and multiple track data or that the multiple track reconstruction algorithm is not working properly. This study will also determine if a scaling factor for events that cannot be salvaged is appropriate. Scaling factors are the standard solution to the multiple track problem.

Figure 5.17 shows the comparison of single to multiple track peaks before the study. The filled red curve shows the timing peak with a single track cut: ( L.tr. $\mathrm{n}==1$ $\& \&$ R.tr. $n==1)$. The blue curve shows the timing peak with a multiple track cut: (L.tr. $\mathrm{n}=1 \& \&$ R.tr. $\mathrm{n}>1$ ) $\mid$ (L.tr. $\mathrm{n}>1$ \&\&R.tr. $\mathrm{R}==1$ ). The multiple track histogram was then scaled to have a similar background plateau level. The peak width and height are similar for the two cuts, as well as the background levels.

Figure 5.18 shows the comparison of particle identification spectra for electrons between events where the timing information could be extracted, and where events are discarded because the timing could not be calculated. Since the discarded events account for 
less than $10 \%$ of the total data stream, the discarded curve has also been scaled up to match the magnitude of the timing peak histogram. The shapes of the good and discarded spectra match qualitatively. Fitting a sum of two gaussians (one for pions and one for electrons) allows a more quantitative comparison of the proportion of electrons in Figure 5.18. The fitting shows $97.3 \%$ of events in the timing peak, and $96.5 \%$ of discarded events are electrons. This trend continues across the kinematic settings in experiment JLab E08-010, with the ratios between good events and discarded events agreeing to within $1 \%$. The differences in electron ratio are accounted for with a systematic uncertainty on to multiple track efficiency factor, adding around $0.1 \%$ to the total systematics of the spectrometer cross section.

The matching shapes in Figures 5.17 and 5.18 indicates that scaling would be appropriate as there is little difference between the two data sets in regards to coincidence timing. The multiple track analysis is not yet complete in Figure 5.17, so the slight slope to the multiple track (blue) background plateau is not a concern. Issues with the shape may be a result of improper selection of hits or tracks. The results after the analysis can be seen in Figure 5.20 where the slope in the background plateau is gone.

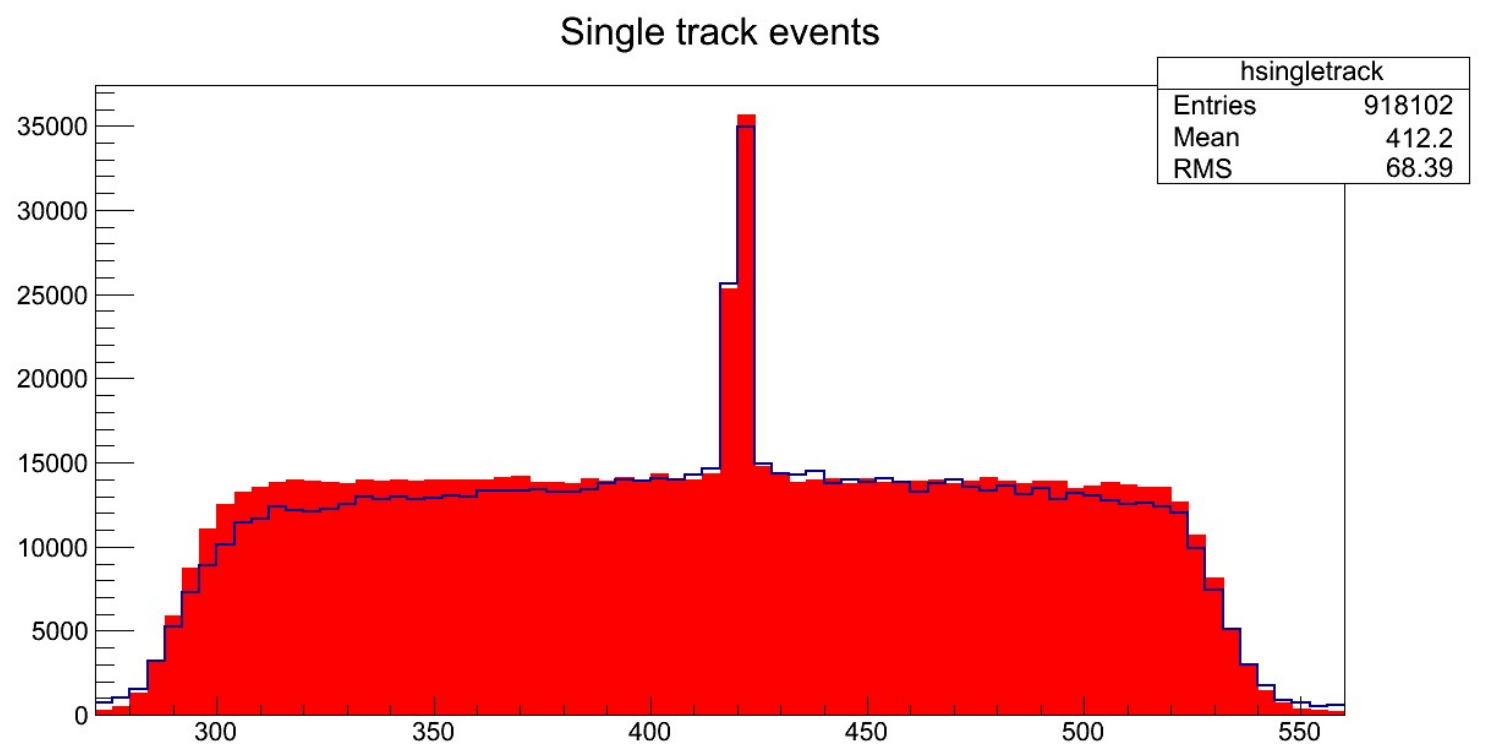

Figure 5.17: Filled Red: single track cut cTOF spectrum. Blue: multi track cut cTOF spectrum scaled to match BG plateau height.

As mentioned previously, there are two sources of event multiplicity: the VDCs contribute tracks and the scintillating panels contribute hits as summarized in Figure 5.19. 


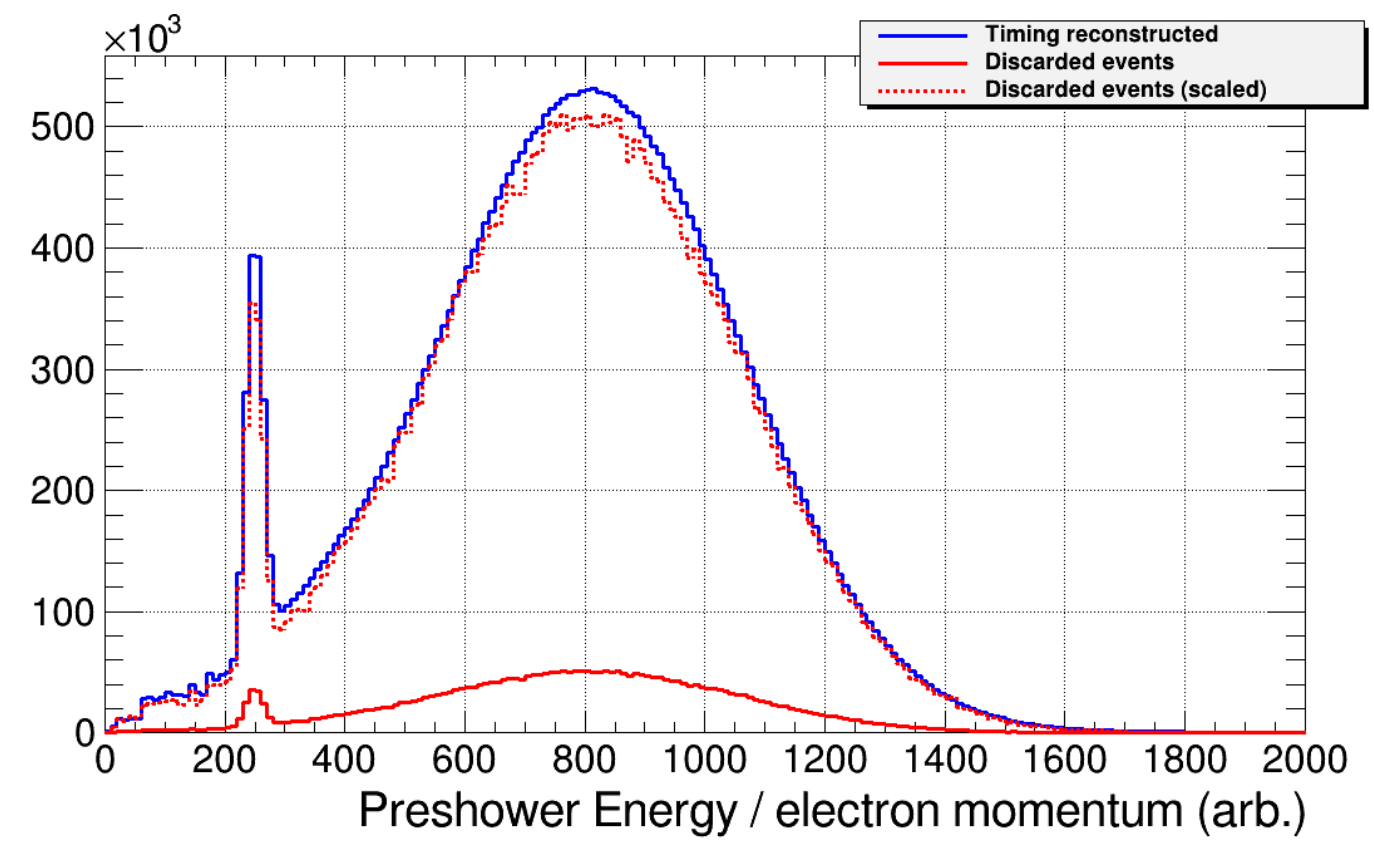

Figure 5.18: Plot comparing the electron particle identification spectrum (energy deposited in the preshower layer divided by electron momentum) for events in the timing peak, and discarded multiple track events.

Perhaps the best way to visualize how these two sources of multiplicity interact is to work through an example. Suppose that a given event has two tracks (labeled 1 and 2, respectively) reported by the VDCs in one arm. Each track will work its way through the detector and hit a panel in the S2 plane where PMTs report timing hits. For now assume that the two tracks hit different panels. The panel hit by track 1 reports two times and the panel hit by track 2 reports 3 times. Now the multiplicities are two for track 1 and three for track 2 . This is because the track information (momentum and position) can be associated with any of the times reported from the panel that track hit. Thus the total multiplicity for this arm is 5 (i.e. one of $1 \mathrm{a}, 1 \mathrm{~b}, 2 \mathrm{a}, 2 \mathrm{~b}$, and $2 \mathrm{c}$ ). The problem of selecting the proper time from these gets complicated quickly if the other arm has similar multiplicity to this example.

The problem gets complicated when the two tracks do not hit separate panels. The panel number hit by the track is the strongest link between the two detectors. When confusion arises here the link becomes tenuous, and so such events are left out of this analysis.

The multi-hit PMTs included in the S2 plane panels have a characteristic response structure to their TDC output[75]. The characteristic response spectrum is found by selecting a clean sample of single track events that have only one hit in the PMT: e.g. (L.tr.n==1 


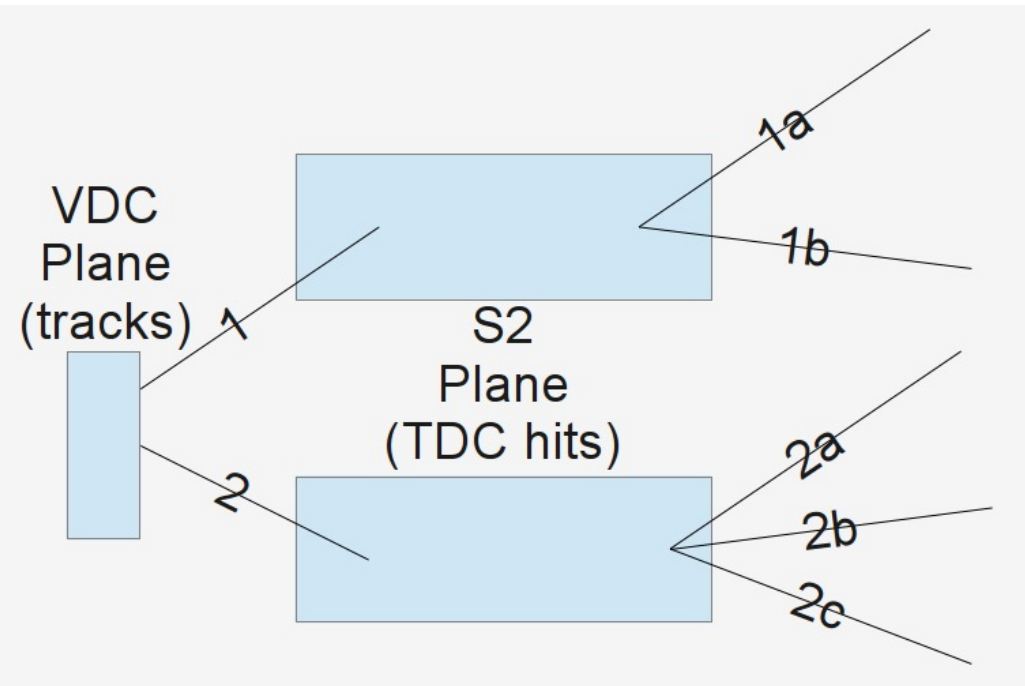

Figure 5.19: Example demonstrating multiplicity contributions from tracks in the VDC and hits in the S2 plane PMT TDC readout.

$\& \&$ L.s2.nrmhit $[\mathrm{L} . \mathrm{s} 2 \cdot \operatorname{trpad}[0]]==1$ ). Find the hit with a response that is closest to this peak value for each event.

This method unambiguously reduces the multiplicity of an event to the number of tracks in that arm. More details about selecting the proper hit value can be found in section 3 of JLab Hall A Tech Note JLAB-TN-05-42 by Mazouz, Bertin, and Voutier.

Once the proper hit for each track has been identified, one can compute the coincidence time with the other arm for each pair of tracks. Our experiment has very little data that has multiple tracks in both arms, so this step is trivial.

The final coincidence time spectrum is shown in Figure 5.20. The red filled line is the result with a single track cut. The blue line is the result with multiple tracks included. The number of events excluded from the analysis is also tracked. The analyzed data is scaled up to account for those events for which a proper timing reconstruction could not be completed.

In summary, of the 25.69 percent of events that were under analysis here, 18.25 percent (over two thirds of the events in question) were recovered and 7.44 percent were not. This makes our total tracking efficiency factor over 90 (up from 72) percent for this kinematic setting. 
single track events

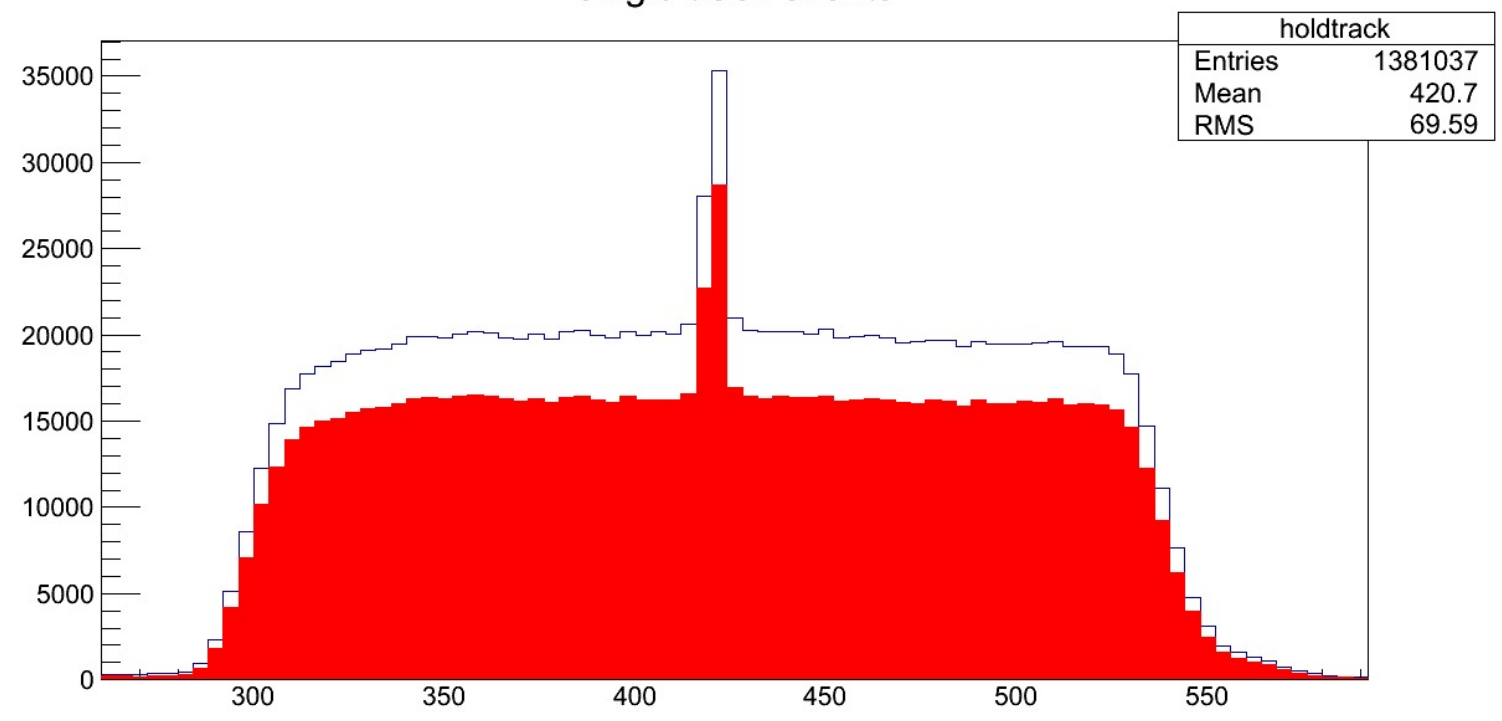

Figure 5.20: Filled Red: single track cut cTOF spectrum. Blue: Complete cTOF spectrum after multi-track analysis.

\subsection{Efficiencies}

A collection of correction factors are applied to the JLab data as efficiencies. These are all calculated on a run by run basis, though averaged for presentation in Table 5.3.

Computer deadtime (DT) is the proportion of events missed due to the computer processing a previous event. Also known as livetime $(L T=1-D T)$. The uncertainty on this value is statistical in nature. The computer livetime at JLab is calculated via:

$$
L T=\frac{N_{T} \cdot P S_{T}}{S_{T}}
$$

Where $N_{T}$ is the total number of events in the data stream for a trigger, $S_{T}$ is the number of scaler counts for the trigger, and $P S_{T}$ is the prescale factor for that trigger.

The computer livetime correction is applied to the data yield $\left(Y_{d a t}\right)$ as an inverse scaling factor.

$$
Y_{d a t} \rightarrow Y_{d a t} / L T
$$

Electronic deadtime is often negligible in JLab experiments. It is calculated from the computer deadtime and the combined electronic and computer deadtime that is measured with a pulser (trigger 8) at JLab. Typical values during the JLab experiment were less than 
$0.5 \%$.

$$
\begin{aligned}
E C D T & =E D T \cdot C D T \\
E D T & =E C D T / C D T
\end{aligned}
$$

Trigger efficiency is the efficeincy of the triggers in each arm at JLab. Calculated by comparing the two trigger types in each arm:

$$
\varepsilon_{L}=\frac{S_{3}}{S_{3}+S_{4}}
$$

The contributions from the end caps at JLab are computed from dummy cell runs. The dummy target has two foils representing the cell walls. The dummy run data is scaled for the thickness of the foils compared to target cell walls, and also the cumulated charge of the run for the data compared to the dummy target runs. This correction is made on a run by run basis. The number of events that pass the analysis cuts from the dummy runs is subtracted from the events passing the same cuts in the data before the cross section is computed.

Most of these efficiency calculations are not required in the MAMI analysis. Lumi++, the luminosity calculating program used at MAMI A1 has computer and electronic deadtimes, as well as tracking and trigger efficiencies built in. Dummy runs were not performed at MAMI. The target cell end caps are cut out of the data stream for that part analysis.

Figure 5.21 shows the phase space overlap at $Q^{2}=0.09(\mathrm{GeV} / \mathrm{c})^{2}$ for the sequential proton azimuthal angle $\phi_{p q}^{*}=0^{\circ}$ and $180^{\circ}$ measurements. The phase space cuts listed in Table 5.4, are well within the overlap region. Corresponding plots for $Q^{2}=0.04$ and $Q^{2}=0.13$ are qualitatively similar. 

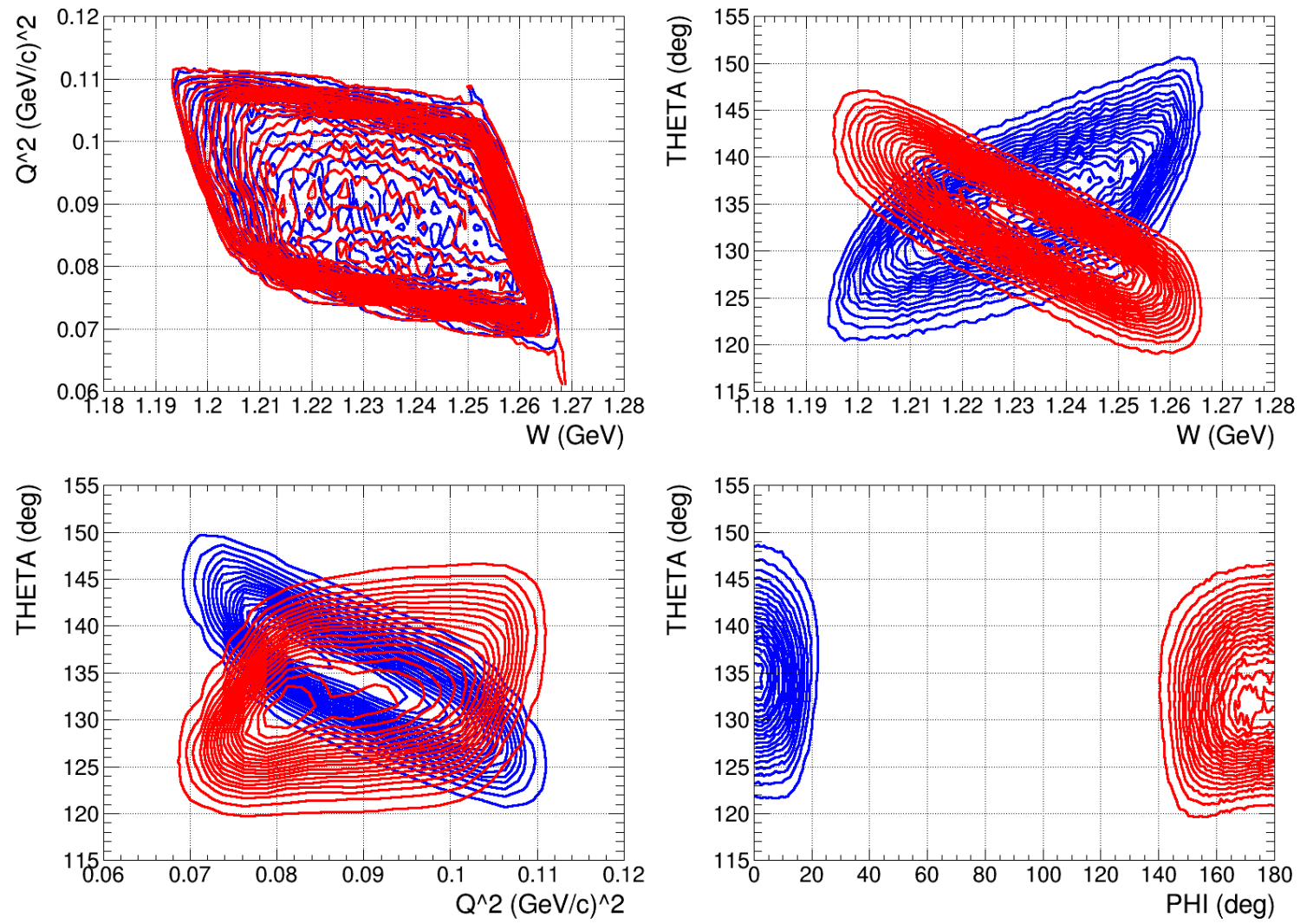

Figure 5.21: Phase space overlap plots for inside and outside kinematics at $\mathrm{Q}^{2}=0.09$ $(\mathrm{GeV} / \mathrm{C})^{2}$. The panels show $\mathrm{Q}^{2}$ vs $\mathrm{W}, \theta_{p q}^{*}$ vs $\mathrm{W}, \theta_{p q}^{*}$ vs $\mathrm{Q}^{2}$, and $\theta_{p q}^{*}$ vs $\phi_{p q}^{*}$. 
Table 5.3: Efficiencies and correction factors at JLab. All corrections are presented as a percentage of the cross section. Uncertainties are given as a percentage of the correction. Kinematics with asterisk denote $15 \mathrm{~cm}$ target. All others use $4 \mathrm{~cm}$ target. The density correction shown is the average across all runs. Corrections are made individually for each run according to the average beam current.

\begin{tabular}{|l|c|c|c|c|}
\hline Kin. & MT & Trig. & LT & Proton Abs. \\
\hline 1 & $7 \pm 0.1$ & $1 \pm 0.2$ & $24 \pm 0.1$ & 1.3 \\
\hline 2 & $7 \pm 0.2$ & $1 \pm 0.2$ & $22 \pm 0.1$ & 2.2 \\
\hline 3 & $9 \pm 0.2$ & $1 \pm 0.2$ & $26 \pm 0.1$ & 1.1 \\
\hline 5 & $11 \pm 0.1$ & $2 \pm 0.2$ & $11 \pm 0.1$ & 1.1 \\
\hline 6 & $7 \pm 0.1$ & $1 \pm 0.2$ & $4 \pm 0.1$ & 2.0 \\
\hline 7 & $9 \pm 0.1$ & $2 \pm 0.2$ & $5 \pm 0.1$ & 0.9 \\
\hline $8 S$ & $7 \pm 0.1$ & $1 \pm 0.2$ & $3 \pm 0.1$ & 1.1 \\
\hline 11 & $7 \pm 0.1$ & $1 \pm 0.2$ & $3 \pm 0.1$ & 1.9 \\
\hline 12 & $6 \pm 0.2$ & $1 \pm 0.2$ & $2 \pm 0.1$ & 0.9 \\
\hline $8 L^{*}$ & $10 \pm 0.1$ & $2 \pm 0.2$ & $10 \pm 0.1$ & 1.1 \\
\hline $9^{*}$ & $10 \pm 0.1$ & $1 \pm 0.2$ & $11 \pm 0.1$ & 1.4 \\
\hline $10^{*}$ & $11 \pm 0.1$ & $2 \pm 0.2$ & $11 \pm 0.1$ & 0.9 \\
\hline $13^{*}$ & $10 \pm 0.1$ & $2 \pm 0.2$ & $9 \pm 0.1$ & 1.0 \\
\hline $14^{*}$ & $11 \pm 0.1$ & $2 \pm 0.2$ & $11 \pm 0.1$ & 1.0 \\
\hline
\end{tabular}

\subsection{Phase space and Simulation}

The phase space acceptance for the experiment was a complex multi-dimensional volume (see Figure 5.21). A Monte Carlo simulation was used to calculate the phase space consistently across all kinematic settings. The choice of cuts to define the limits of the acceptance phase space is arbitrary, and based on the geometry of the detector. To ensure our phase space was consistent across kinematics, and stable with respect to variations of the boundaries, the cross section was computed with a systematically varied set of cuts, and the stability of the result was studied.

A standard set of cuts was identified from the acceptance shown by the data stream. A basic cut based on the known acceptance for the spectrometers was used for each spectrometer For the kinematic variables $Q^{2}$ and $\theta_{p q}^{*}$, the standard cut was the point were the data yield at each edge fell to half the peak value. The cuts on $\mathrm{W}, Q^{2}$ and $\theta_{p q}^{*}$ were found to limit $\phi_{p q}^{*}$ naturally, so no cut was applied directly to $\phi_{p q}^{*}$. For W, a standard central bin of $16 \mathrm{MeV}$ total width centered at the $\Delta$ resonance $(1232 \mathrm{MeV})$. The cross section in this regime is known to be quite smooth in $\mathrm{W}$ from the model calculations, and the bin is wide 
enough to accept a significant proportion of the detected events. A cut fraction value is used to modify these cuts in concert (i.e. a cut fraction of 0.9 corresponds to the $\mathrm{W}$ cut width shrinking to $12.8 \mathrm{MeV}$ at the same time $Q^{2}$ and $\theta_{p q}^{*}$. are all reduced $10 \%$ ). Figure 5.22 shows the stability of the cross section across a wide range of cuts in this analysis, and a final cut fraction value of 0.8 was chosen to represent the final cut positions listed in Table 5.4. The total variation between cuts is less than $\pm 2 \%$ varying from $20-120 \%$ of the base cuts, with less than $\pm 0.5 \%$ in the region of the final cut.

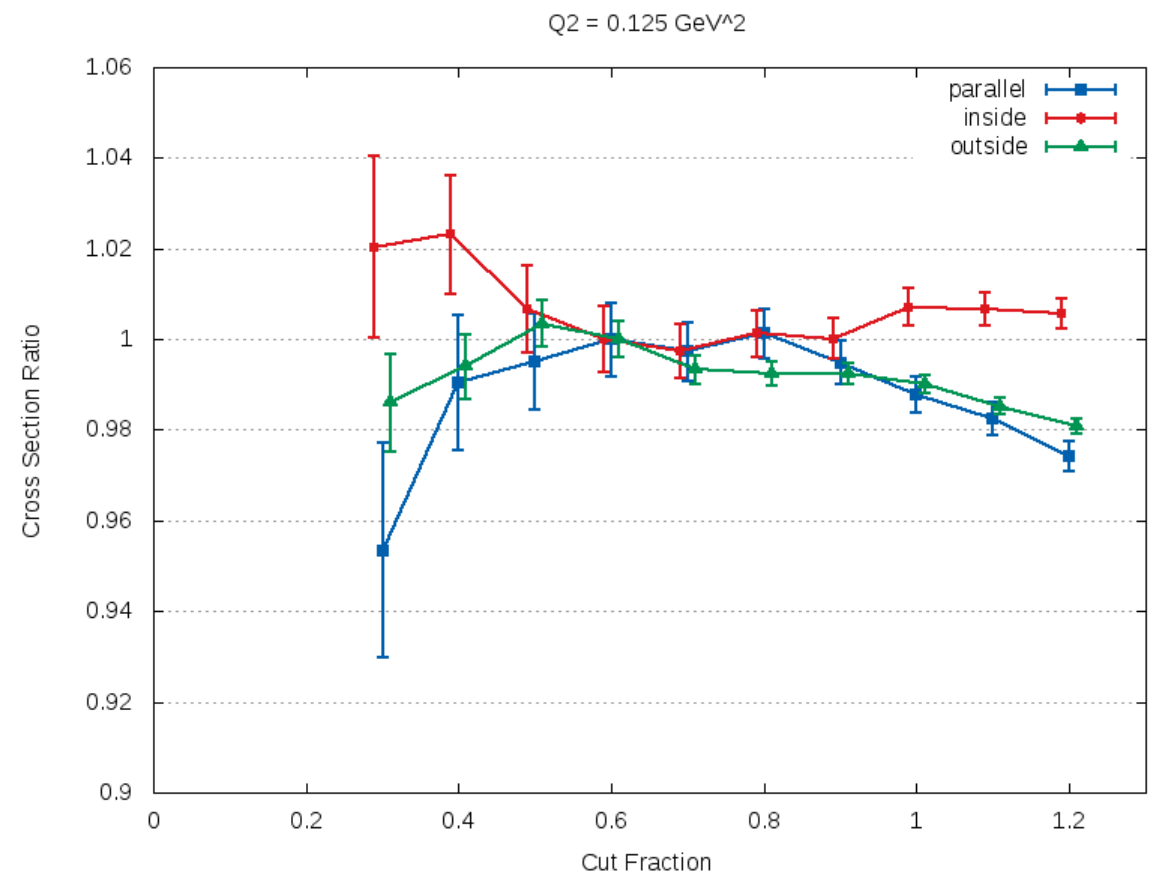

Figure 5.22: Normalized cross section vs cut fraction

A similar process was used to define the final cuts at MAMI. The final kinematics cuts used are shown in Table 5.4.

MCEEP [76], a Monte Carlo simulation of the JLab HRS geometry and e,ep' scattering was integral to understanding the geometry of the spectrometers. MCEEP randomly samples the acceptance of the HRS, projecting the path of a particle through the spectrometer while checking several aperatures and fields are passed successfully. MCEEP weights each event for kinematic factors such as the photon flux $(\Gamma)$ and lab to center-of-mass Jacobian, as well as probabilistic adjustments like radiative corrections and energy loss. Additionally, look up tables were created for four models (DMT [31], MAID [33], SAID [35], and Sato Lee [30]), and used as weighting factors inside MCEEP to calculate simulated yields for 
Table 5.4: Cut definitions for the kinematic variables $\mathrm{W}, Q^{2}$, and $\theta_{p q}^{*}$. The cut widths are common among all kinematics sharing the same central value. For $\theta_{p q}^{*}$ there are two widths, with all non-zero central values sharing the same cut.

\begin{tabular}{|c|c|c|}
\hline Variable & Nominal Value & Range \\
\hline \multicolumn{3}{|c|}{ JLab } \\
\hline $\mathrm{W}(\mathrm{MeV})$ & 1170 & $1163.6-1176.4$ \\
\hline $\mathrm{W}(\mathrm{MeV})$ & 1200 & $1193.6-1206.4$ \\
\hline $\mathrm{W}(\mathrm{MeV})$ & 1232 & $1225.6-1238.4$ \\
\hline$Q^{2}(\mathrm{GeV} / \mathrm{c})^{2}$ & 0.040 & $0.032-0.048$ \\
\hline$Q^{2}(\mathrm{GeV} / \mathrm{c})^{2}$ & 0.090 & $0.0796-0.1004$ \\
\hline$Q^{2}(\mathrm{GeV} / \mathrm{c})^{2}$ & 0.130 & $0.118-0.142$ \\
\hline$\theta_{p q}^{*}\left({ }^{\circ}\right)$ & 0 & $0-14.4$ \\
\hline$\theta_{p q}^{*}\left({ }^{\circ}\right)$ & other & other \pm 4 \\
\hline \multicolumn{3}{|c|}{ MAMI } \\
\hline $\mathrm{W}(\mathrm{MeV})$ & 1232 & $1222-1242$ \\
\hline$Q^{2}(\mathrm{GeV} / \mathrm{c})^{2}$ & 0.2 & $0.18-0.22$ \\
\hline$\theta_{\gamma \gamma}^{*}\left({ }^{\circ}\right)$ & 128 or 138 & \pm 4.5 \\
\hline$\phi_{\gamma \gamma}^{*}\left({ }^{\circ}\right)$ & 0 or 180 & \pm 10 \\
\hline$Z_{p o s}(\mathrm{~mm})$ & 0 & $-20-20$ \\
\hline$\varepsilon$ & 0.77 & $0.75-0.79$ \\
\hline \multicolumn{3}{|c}{} \\
\hline
\end{tabular}


comparison with detected yields from the experiment. The yields from data and simulation undergo the same kinematic selection process such that direct comparisson is possible.

Figure 5.23 shows the agreement between data and simulation in the acceptance variables.
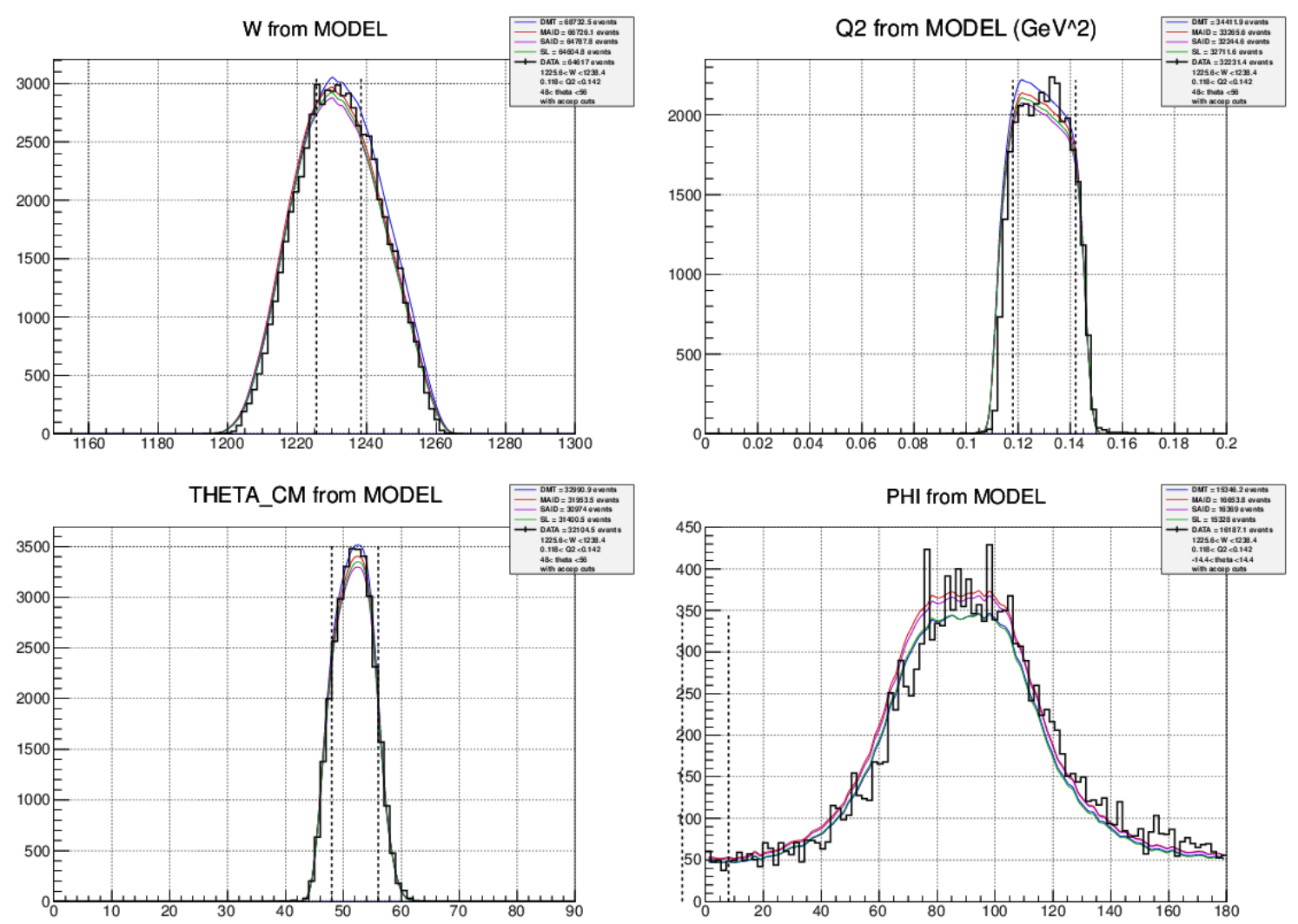

Figure 5.23: Comparisson of data and simulation for $\mathrm{W}, Q^{2}, \theta_{p q}^{*}$, and $\phi_{p q}^{*}$ yields in spectrometer. 


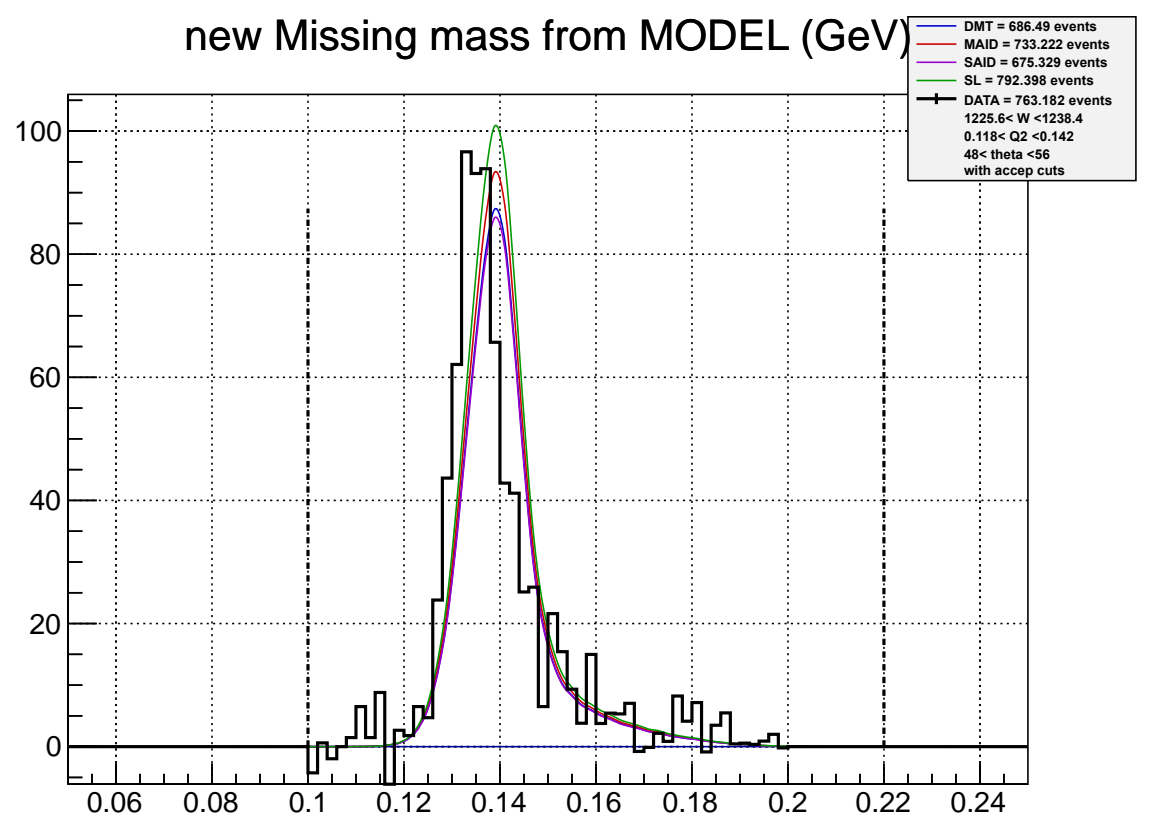

Figure 5.24: Comparisson of data and simulation for reconstructed mass of undetected pion.

\subsection{Bin centering}

The experiment measures data averaged across the multidimensional phase space acceptance of the spectrometer, while the models give point predictions. In order to compare the two, the data was collapsed to the kinematical central point for comparisson. This collapse is performed by calculating the average of the model cross section across the phase space acceptance of the data via Monte Carlo simulation. It is important the data and simulation generally have the same shape for this step, which can be seen in Figure 5.23. This step is cross checked with tighter phase space cuts showing a stable central point cross section being extracted, as well as the bin centering ratio of the simulation converging to one. This correction is typically of the order $5 \%$, and adds to the systematic uncertainty via computation of the RMS deviation of the final results calculated with several models.

\subsection{Fitting}

The multipole amplitudes and polarizability results were extracted by fitting model parameters to the cross sections and asymmetries. The fitting was accomplished through least 
squares regression analysis of parameter models. This was done first using a Monte Carlo technique proposed in [62], and then with the Minuit [63] Migrad fitting algorithm.

The Monte Carlo fitting process uses random values for the input parameters: multipole amplitudes for $l=0$ to $l=5$ in the case of JLab, and the C2 amplitude, $\Lambda_{\alpha}$, and $\Lambda_{\beta}$ at MAMI. A flat probability distribution is used for these to allow a test for which parameters contribute significantly to the fit before running Migrad. The randomly chosen parameters are used to calculate the predicted cross section and calculate a weighted $\chi^{2}$ value according to:

$$
\chi^{2}=\frac{\sum\left(\sigma_{\text {pred }}-\sigma_{\text {data }}\right)^{2} / \Delta \sigma_{\text {data }}^{2}}{\sum 1 / \Delta \sigma_{\text {data }}^{2}}
$$

This results in a model independent cross section analysis for pion electroproduction. The Dispersion Relation model used to calculate the predicted cross sections $\left(\sigma_{\text {pred }}\right)$ uses fixed multipole amplitudes from MAID and fixed proton form factors, so the result is not model independent despite tuning the DR mass parameters.

This Monte Carlo method of fitting is used to find significant amplitudes, check for local minima, and check the size of the $\chi^{2}$ minima in the search parameter space. Once the phase space of parameters is unerstood, tuned parameters are used in the Migrad minimization routine of Minuit [63] to do a model dependent fit for each model (DMT, MAID, SAID, and Sato Lee for pion electroproduction, and DR for VCS). Finally the Minos function is used to compute values for fit uncertainties.

Systematic uncertainties in the fitting are found through variation of the cross sections being fit. The root mean square deviation of the resultant set is used as the systematic uncertainty. The model uncertainties are computed in a similar fashion for pion electroproduction as a RMS deviation calculated on extractions from the four models.

\subsection{Systematic uncertainties}

The final systematic uncertainties in the cross sections for the two experiments are summarized in Tables 5.5 and 5.6. Both experiments see total systematic uncertainties $2.5 \%$, which is comparable to similar experiments with these apparatus. The systematic uncertainties in the results calculated from the cross section values are estimated from the root 
mean square variation of the extraction when the cross sections are each independently varied by their associated systematic uncertainty.

Table 5.5: Summary of systematic and model uncertainties at JLab.

\begin{tabular}{|c|c|}
\hline Uncertainty & Size (\%) \\
\hline Target Density & $0.25-1.0$ \\
\hline Target Length & 0.1 \\
\hline Beam Current & 0.5 \\
\hline Proton Absorption & 0.25 \\
\hline Particle Identification & 0.1 \\
\hline Live time correction & $<0.5$ \\
\hline Momentum and Angular Resolution & 1.5 \\
\hline Phase space cuts & $0.2-1.4$ \\
\hline Multiple Tracks & $<0.2$ \\
\hline Sum & $2.2-2.6$ \\
\hline
\end{tabular}

Table 5.6: Summary of systematic and model uncertainties at MAMI.

\begin{tabular}{|c|c|}
\hline Uncertainty & Size (\%) \\
\hline Luminosity & 2 \\
\hline Detector Efficiency Correction & 1 \\
\hline Momentum and Angular Resolution & 1 \\
\hline Proton Absorption & 0.1 \\
\hline Particle Identification & $<0.1$ \\
\hline Live time correction & $<0.5$ \\
\hline Phase space cuts & 1 \\
\hline Sum & 2.7 \\
\hline
\end{tabular}




\section{CHAPTER 6}

\section{RESULTS AND DISCUSSION}

\subsection{JLab}

Data were collected at 13 kinematic settings in the JLab Hall A E08-010 experiment. The kinematics were broken up into several sections to allow different extractions. First, three setups each are defined at $Q^{2}=0.04$ and $0.09(\mathrm{GeV} / \mathrm{c})^{2}$ to allow extraction of the multipoles. Seven kinematics are run at $Q^{2}=0.13(\mathrm{GeV} / \mathrm{c})^{2}$. Five at the $\Delta(1232)$ resonance for precise multipole extraction, and two additional settings at lower $\mathrm{W}$ values (1200 and $1170 \mathrm{MeV})$.

\subsubsection{Cross Sections and Asymmetries}

Spectrometer cross sections for each setting are summarized in Table 6.1. Note the high precision of the measurements with statistical uncertainties below $0.5 \%$, model uncertainties around $1 \%$, and systematic uncertainties controlled to the $2.5 \%$ level.

The parallel cross sections $\left(\sigma_{0}^{\|}=\sigma_{T}+\varepsilon \cdot \sigma_{L}\right)$ taken along the momentum transfer direction have been identified in Table 6.2. These are easily compared with other experiments by scaling for the epsilon and $\mathrm{Q}^{2}$ dependence using models. In particular, our measurement at $\mathrm{Q}^{2}=0.13(\mathrm{GeV} / \mathrm{c})^{2}$ and $\varepsilon=0.8505(11.3 \pm 0.06 \mathrm{stat} \pm 0.05 \mathrm{model} \pm 0.25 \mathrm{sys} \mu \mathrm{b} / \mathrm{sr})$ agrees within uncertainties with the scaled point from [38] measured at $\mathrm{Q}^{2}=0.127(\mathrm{GeV} / \mathrm{c})^{2}$ and $\varepsilon$ $=0.707$ which scales to $11.1 \pm 0.1$ stat \pm 0.36 sys $\mu \mathrm{b} / \mathrm{sr}$.

Additionally, the evolution of the cross sections can be compared with models in both $\mathrm{W}$ and in $\mathrm{Q}^{2}$. Figure 6.1 shows the four models all qualitatively describing the slight downward trend in parallel cross section as momentum transfer is decreased. 
Table 6.1: Measured pion electroproduction cross sections from JLab E08-010. Uncertainties are statistical, model, and systematic.

\begin{tabular}{|c|c|c|c|c|}
\hline$Q^{2}(\mathrm{GeV} / \mathrm{c})^{2}$ & $\mathrm{~W}(\mathrm{MeV})$ & $\theta_{p q}^{*}\left({ }^{\circ}\right)$ & $\phi_{p q}^{*}\left({ }^{\circ}\right)$ & $d \sigma / d \Omega(\mu b / s r)$ \\
\hline 0.04 & 1232 & 0 & - & $10.3 \pm 0.03 \pm 0.03 \pm 0.23$ \\
\hline 0.04 & 1232 & 35 & 0 & $8.86 \pm 0.03 \pm 0.07 \pm 0.21$ \\
\hline 0.04 & 1232 & 35 & 180 & $12.9 \pm 0.02 \pm 0.11 \pm 0.28$ \\
\hline 0.09 & 1232 & 0 & - & $10.7 \pm 0.05 \pm 0.02 \pm 0.25$ \\
\hline 0.09 & 1232 & 47 & 0 & $10.4 \pm 0.04 \pm 0.05 \pm 0.23$ \\
\hline 0.09 & 1232 & 47 & 180 & $16.2 \pm 0.03 \pm 0.14 \pm 0.37$ \\
\hline 0.13 & 1232 & 0 & - & $11.3 \pm 0.06 \pm 0.05 \pm 0.25$ \\
\hline 0.13 & 1232 & 32 & 0 & $9.77 \pm 0.05 \pm 0.04 \pm 0.22$ \\
\hline 0.13 & 1232 & 32 & 180 & $16.2 \pm 0.06 \pm 0.15 \pm 0.40$ \\
\hline 0.13 & 1232 & 52 & 0 & $11.5 \pm 0.05 \pm 0.04 \pm 0.28$ \\
\hline 0.13 & 1232 & 52 & 180 & $19.4 \pm 0.05 \pm 0.19 \pm 0.47$ \\
\hline 0.13 & 1170 & 0 & - & $9.12 \pm 0.05 \pm 0.11 \pm 0.24$ \\
\hline 0.13 & 1200 & 0 & - & $13.7 \pm 0.07 \pm 0.11 \pm 0.36$ \\
\hline
\end{tabular}

Table 6.2: Measured pion electroproduction parallel cross sections from JLab E08-010. Uncertainties are statistical, model, and systematic.

\begin{tabular}{|c|c|c|}
\hline$Q^{2}(\mathrm{GeV} / \mathrm{c})^{2}$ & $\mathrm{~W}(\mathrm{MeV})$ & $d \sigma / d \Omega(\mu b / s r)$ \\
\hline 0.04 & 1232 & $10.3 \pm 0.03 \pm 0.03 \pm 0.22$ \\
\hline 0.09 & 1232 & $10.7 \pm 0.05 \pm 0.02 \pm 0.25$ \\
\hline 0.13 & 1232 & $11.3 \pm 0.06 \pm 0.05 \pm 0.25$ \\
\hline 0.13 & 1170 & $9.12 \pm 0.05 \pm 0.11 \pm 0.24$ \\
\hline 0.13 & 1200 & $13.7 \pm 0.07 \pm 0.11 \pm 0.36$ \\
\hline
\end{tabular}




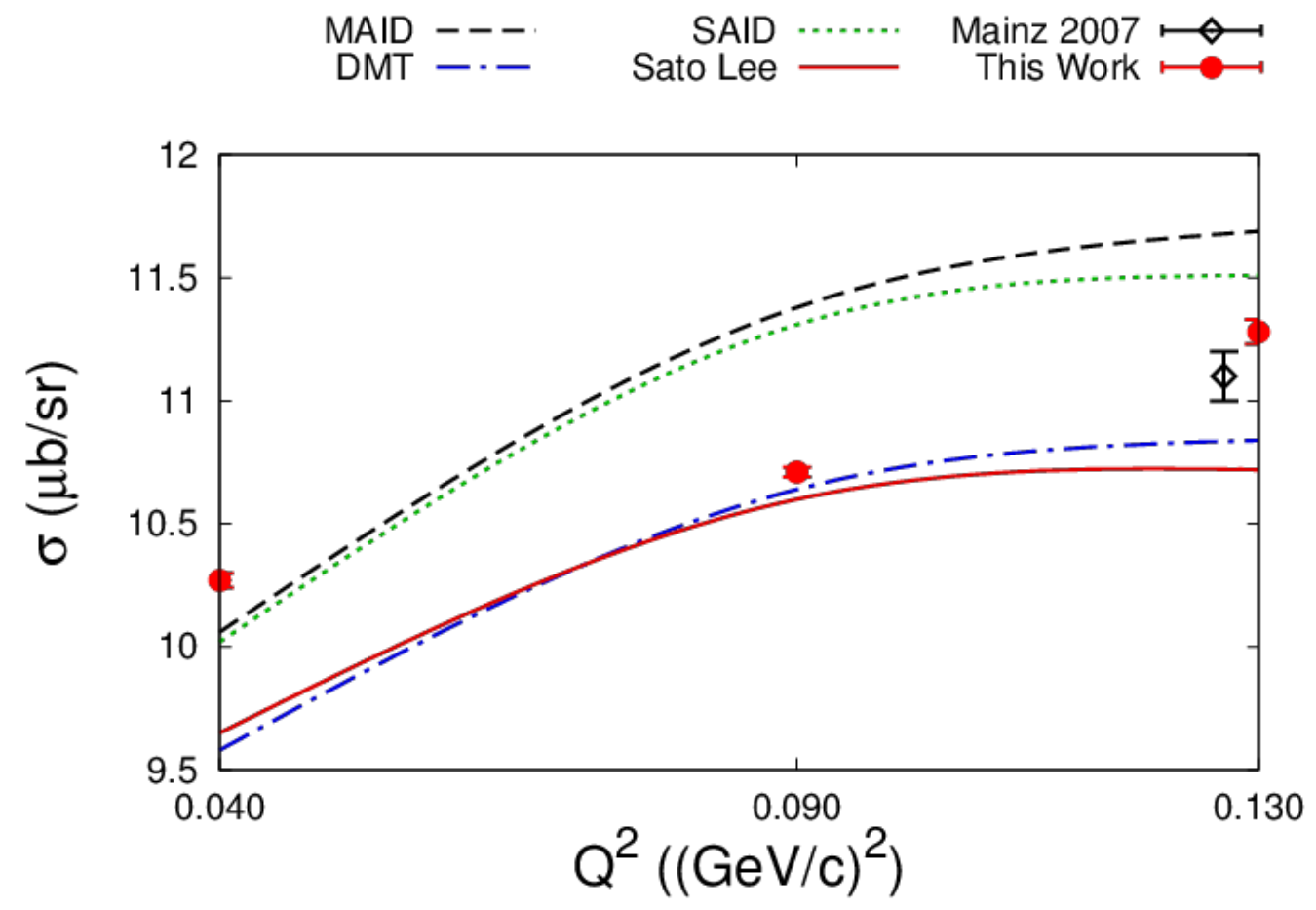

Figure 6.1: $\mathrm{Q}^{2}$ evolution of parallel cross section of JLab E08-010 at $\mathrm{W}=1232 \mathrm{MeV}$. The Mainz point is from [38]. Errorbars show statistical uncertainty. 
The performance of the models in a $\mathrm{W}$ scan from 1170 to $1232 \mathrm{MeV}$. In Figure 6.2, as seen previously [38], the Sato Lee [30] model does a good job describing the peak in W. The phenomenological models MAID [33] and SAID [35] overshoot the peak, and the DMT [31] model is slightly low.
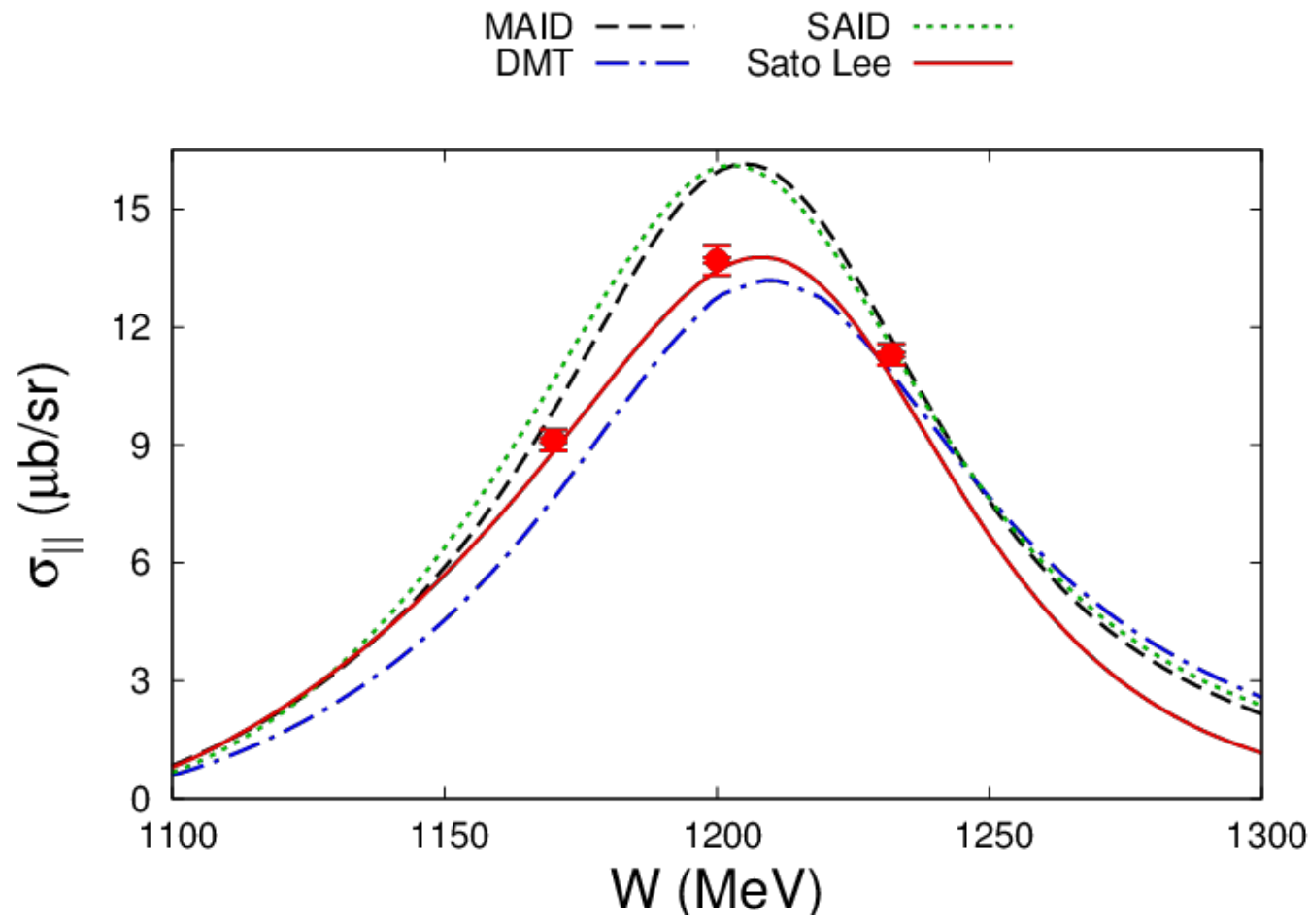

Figure 6.2: $\mathrm{W}$ evolution of parallel cross section of JLab E08-010 at $\mathrm{Q}^{2}=0.13(\mathrm{GeV} / \mathrm{c})^{2}$. Errorbars show statistical uncertainty.

Figures 6.3, 6.4, and 6.5 show the algebraic extractions from $\sigma\left(\phi_{p q}^{*}=0^{\circ}\right)$ and $\sigma\left(\phi_{p q}^{*}=\right.$ $\left.180^{\circ}\right)$ at each momentum transfer. The top plot in each figure is the azimuthal asymmetry ( $A=\frac{\sigma_{\phi_{p q}=\pi}-\sigma_{\phi_{p q}=0}}{\sigma_{\phi_{p q}=\pi}+\sigma_{\phi_{p q}=0}}$ ) plotted as a percentage. The asymmetry data fall between the SAID and Sato Lee predictions at all three values of momentum transfer. The middle plots show the longitudinal-transverse partial cross section $\left(\sigma_{L T}=\frac{\sigma_{\phi_{p q}=0}-\sigma_{\phi_{p q}=\pi}}{2 \sqrt{2 \varepsilon(1+\varepsilon)}}\right)$. Here, the Sato Lee model correctly predicts the values for the lower two $\mathrm{Q}^{2}$ points, but MAID is a better fit in the $\mathrm{Q}^{2}=0.130(\mathrm{GeV} / \mathrm{c})^{2}$. Finally, the bottom plota show the sum $\left(\sigma_{0}+\varepsilon \cdot \sigma_{T T}=\right.$ $\left.\frac{\sigma_{\phi_{p q}=0}+\sigma_{\phi_{p q}=\pi}}{2}\right)$, where all four models show qualitative agreement with the data, but none are consistent across the entire range of momentum transfer shown. The data can also be found in Table 6.3. For all quantities shown, the statistical uncertainties are calculated algebraically from the cross section statistical uncertainties. Systematic and model uncer- 
tainties are estimated through the root mean square (RMS) variation of extractions varying each spectrometer cross section independently by its associated uncertainty.

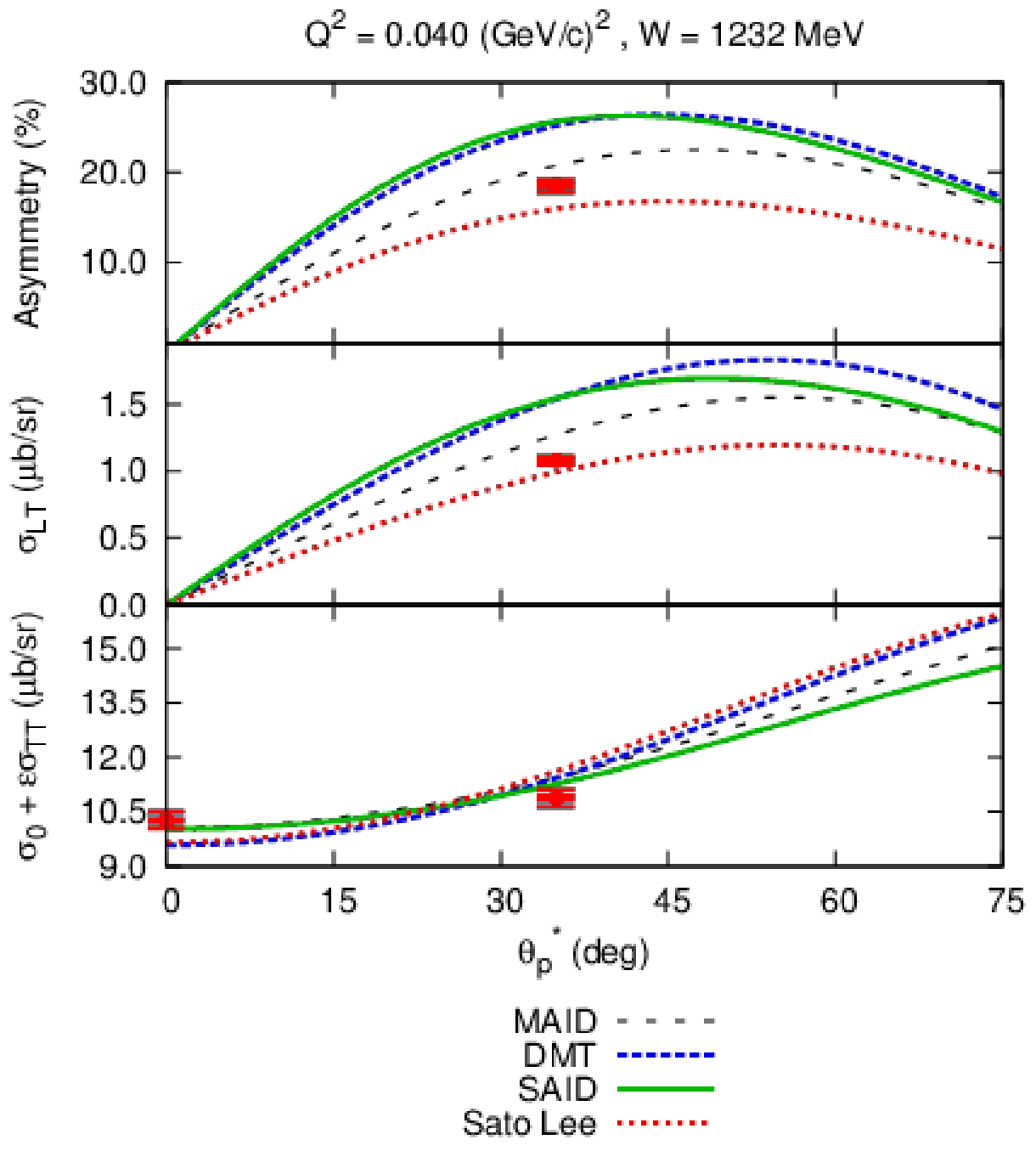

Figure 6.3: Summary plots for algebraic extractions at $\mathrm{Q}^{2}=0.040(\mathrm{GeV} / \mathrm{c})^{2}$. Top panel: Asymmetry vs center of mass proton scattering angle $(\theta)$. Middle Panel: longitudinal-transverse partial cross section vs $\theta$. Bottom panel: $\sigma_{0}+\varepsilon \cdot \sigma_{T T}$ vs $\theta$, note $\sigma_{T T} \rightarrow 0$ as $\theta \rightarrow 0$. Inside errorbars show statistical uncertainty, outside show statistical plus systematic uncertainties added in quadrature. 


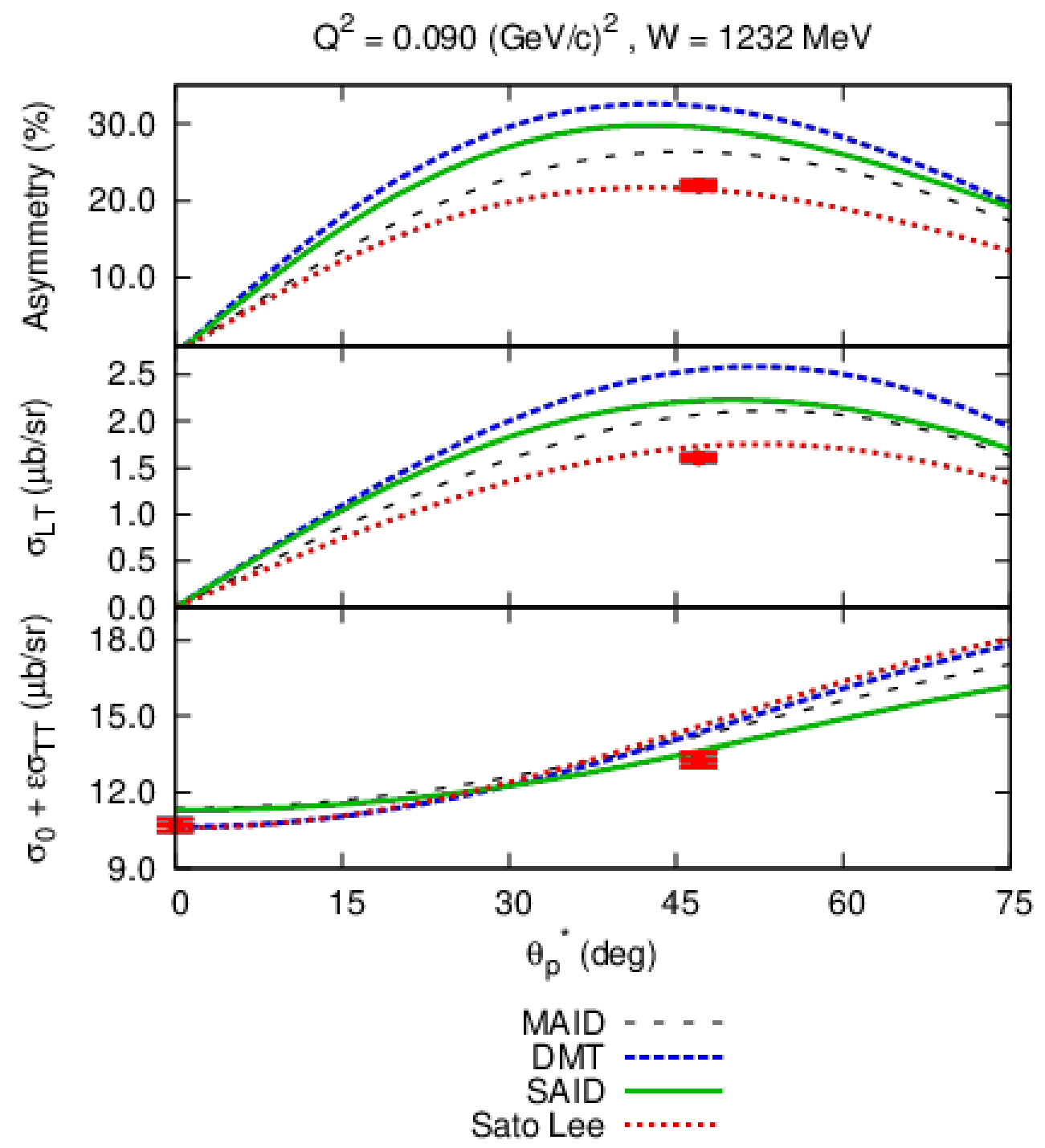

Figure 6.4: Summary plots for algebraic extractions at $\mathrm{Q}^{2}=0.090(\mathrm{GeV} / \mathrm{c})^{2}$. Top panel: Asymmetry vs center of mass proton scattering angle $(\theta)$. Middle Panel: longitudinal-transverse partial cross section vs $\theta$. Bottom panel: $\sigma_{0}+\varepsilon \cdot \sigma_{T T}$ vs $\theta$, note $\sigma_{T T} \rightarrow 0$ as $\theta \rightarrow 0$. Inside errorbars show statistical uncertainty, outside show statistical plus systematic uncertainties added in quadrature. 


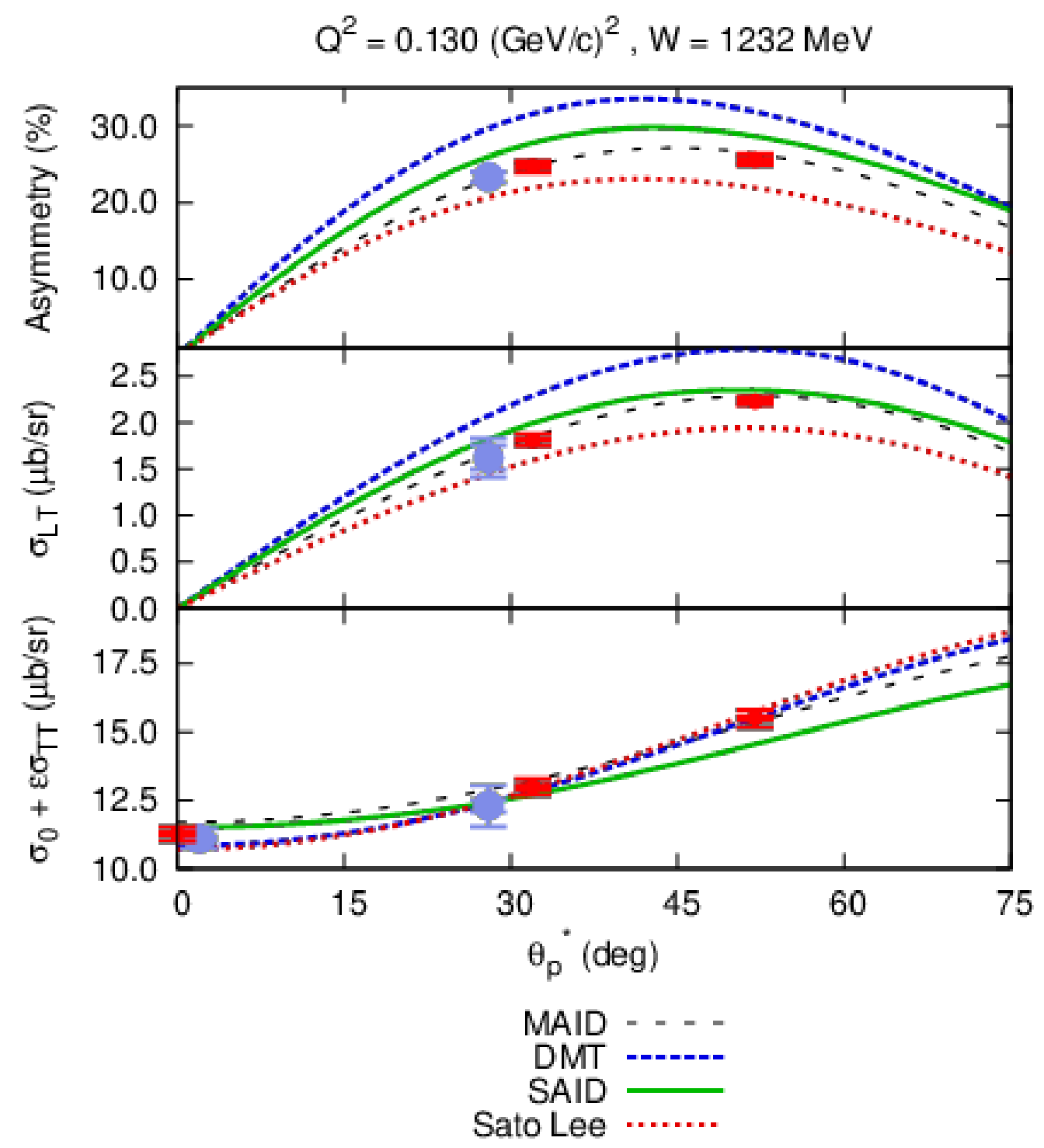

Figure 6.5: Summary plots for algebraic extractions at $\mathrm{Q}^{2}=0.130(\mathrm{GeV} / \mathrm{c})^{2}$. Top panel: Asymmetry vs center of mass proton scattering angle $(\theta)$. Middle Panel: longitudinal-transverse partial cross section vs $\theta$. Bottom panel: $\sigma_{0}+\varepsilon \cdot \sigma_{T T}$ vs $\theta$, note $\sigma_{T T} \rightarrow 0$ as $\theta \rightarrow 0$. Inside errorbars show statistical uncertainty, outside show statistical plus systematic uncertainties added in quadrature. 
Table 6.3: Pion electroproduction asymmetries and partial cross sections from JLab E08-010 measured at the $\Delta$ resonance $(\mathrm{W}=1232 \mathrm{MeV})$. Uncertainties are statistical, model, and systematic.

\begin{tabular}{|c|c|c|}
\hline$Q^{2}(\mathrm{GeV} / \mathrm{c})^{2}$ & $\theta_{p q}^{*}\left({ }^{\circ}\right)$ \\
\hline \multicolumn{3}{|c|}{$d \sigma_{0} / d \Omega+\varepsilon \cdot d \sigma_{T T} / d \Omega(\mu b / s r)$} \\
\hline 0.04 & 35 & $10.7 \pm 0.05 \pm 0.13 \pm 0.19$ \\
\hline 0.09 & 47 & $13.3 \pm 0.06 \pm 0.13 \pm 0.24$ \\
\hline 0.13 & 32 & $13.0 \pm 0.08 \pm 0.13 \pm 0.25$ \\
\hline 0.13 & 52 & $15.5 \pm 0.08 \pm 0.16 \pm 0.28$ \\
\hline \multicolumn{3}{|c|}{$d \sigma_{L T} / d \Omega(\mu b / s r)$} \\
\hline 0.04 & 35 & $1.01 \pm 0.004 \pm 0.01 \pm 0.02$ \\
\hline 0.09 & 47 & $1.61 \pm 0.01 \pm 0.02 \pm 0.03$ \\
\hline 0.13 & 32 & $1.81 \pm 0.01 \pm 0.02 \pm 0.06$ \\
\hline 0.13 & 52 & $2.23 \pm 0.01 \pm 0.02 \pm 0.04$ \\
\hline \multicolumn{3}{|c|}{ Asymmetry $(\%)$} \\
\hline 0.04 & 35 & $17.75 \pm 0.19 \pm 0.58 \pm 0.31$ \\
\hline 0.09 & 47 & $22.05 \pm 0.26 \pm 0.55 \pm 0.14$ \\
\hline 0.13 & 32 & $24.70 \pm 0.28 \pm 0.60 \pm 0.46$ \\
\hline 0.13 & 52 & $25.54 \pm 0.30 \pm 0.66 \pm 0.20$ \\
\hline
\end{tabular}

\subsubsection{Fitted Extractions}

The multipoles are extracted via Minuit [63] fitting of the $M_{1+}^{3 / 2}, E_{1+}^{3 / 2}$, and $S_{1+}^{3 / 2}$ multipoles within the framework of each of the four models. A Monte Carlo fitting method was used to verify that no other multipoles needed fitting. This technique also showed that a two parameter fit was all that was required in the lowest $\mathrm{Q}^{2}$ case, so the $E_{1+}^{3 / 2}$ was fixed at the model prediction for that kinematic. Results of the fits are shown in Table 6.4. For all quantities shown, the statistical uncertainties are calculated in the Migrad routine of Minuit using the cross section statistical uncertainties in the fit. Systematic uncertainties are estimated through the root mean square (RMS) variation of extractions varying each spectrometer cross section independently by its systematic uncertainty. The model uncertainty includes two terms added in quadrature. The first term is calculated according to the systematic uncertainty procedure, and the second is the RMS deviation of the four model fits.

Figure 6.6 shows the $\mathrm{Q}^{2}$ evolution of the $M_{1+}^{3 / 2}$ and $E_{1+}^{3 / 2}$ multipoles measured here along with previous data and theoretical predictions. The $M_{1+}^{3 / 2}$ multipole in particular (bottom plot) is very well known from previous studies [11, 22, 24, 37, 39], and largely determines 
Table 6.4: Pion electroproduction extracted multipoles from JLab E08-010 measured at the $\Delta$ resonance $(\mathrm{W}=1232 \mathrm{MeV})$. Uncertainties are statistical, systematic, and model. The data at $\mathrm{Q}^{2}=0.04(\mathrm{GeV} / \mathrm{c})^{2}$ show insufficient sensitivity to the EMR for a meaningful fit result to be presented.

\begin{tabular}{|c|c|}
\hline$Q^{2}(\mathrm{GeV} / \mathrm{c})^{2}$ & value \pm stat \pm sys \pm model \\
\hline \multicolumn{2}{|c|}{$M_{1+}^{3 / 2}\left(10^{-3} / m_{\pi^{+}}\right)$} \\
\hline 0.04 & $39.40 \pm 0.07 \pm 0.35 \pm 0.54$ \\
\hline 0.09 & $40.40 \pm 0.08 \pm 0.37 \pm 0.41$ \\
\hline 0.13 & $41.50 \pm 0.07 \pm 0.39 \pm 0.46$ \\
\hline \multicolumn{2}{|c|}{ CMR $(\%)$} \\
\hline 0.04 & $-2.9 \pm 0.10 \pm 0.06 \pm 0.62$ \\
\hline 0.09 & $-3.7 \pm 0.10 \pm 0.03 \pm 0.62$ \\
\hline 0.13 & $-4.9 \pm 0.09 \pm 0.08 \pm 0.60$ \\
\hline \multicolumn{2}{|c|}{ EMR $(\%)$} \\
\hline 0.04 & $-1.7 \pm 0.17 \pm 0.03 \pm 0.27$ \\
\hline 0.09 & $-2.4 \pm 0.15 \pm 0.15 \pm 0.24$ \\
\hline 0.13 &
\end{tabular}

the normalization of results. Our data is in good agreement with previous work, and helps solidify the shape taken by the theoretical curves. The $E_{1+}^{3 / 2}$ multipole results (top plot) taken together with previous data indicate a very flat EMR with little structure in the low momentum transfer range shown.

Figure 6.7 shows the extracted CMR results in context with previous world data and theoretical predictions. The highest $\mathrm{Q}^{2}$ data point measured in this experiment at $\mathrm{Q}^{2}=$ $0.130(\mathrm{GeV} / \mathrm{c})^{2}$ agrees well with previous measurements. Moving left from there, our data track the large- $\mathrm{N}_{c}$ [77] and Sato-Lee [30] predictions, showing for the first time a change in the trend of CMR data at momentum transfer below $\mathrm{Q}^{2}=0.1(\mathrm{GeV} / \mathrm{c})^{2}$. During our analysis, we were able to discuss methods with the author of the only other data point in this region [24]. This point was measured at $\mathrm{W}=1221 \mathrm{MeV}$, and scaled to the $1232 \mathrm{MeV}$ of the other values plotted using models. There is significant model variation in this range, which introduces uncertainties not typically plotted in the CMR summary figure. Using the wide acceptance in $\mathrm{W}$, and our large data set, we were able to repeat our analysis of our lowest $\mathrm{Q}^{2}$ point using the methods of [24], and found our point fell approximately $1 \%$ lower across all four models (i.e. at $-4 \%$ ). This value agrees within systematic uncertainties with the point from [24]. Should the data from [24] permit, a new analysis without the scaling in $\mathrm{W}$ may be appropriate. 

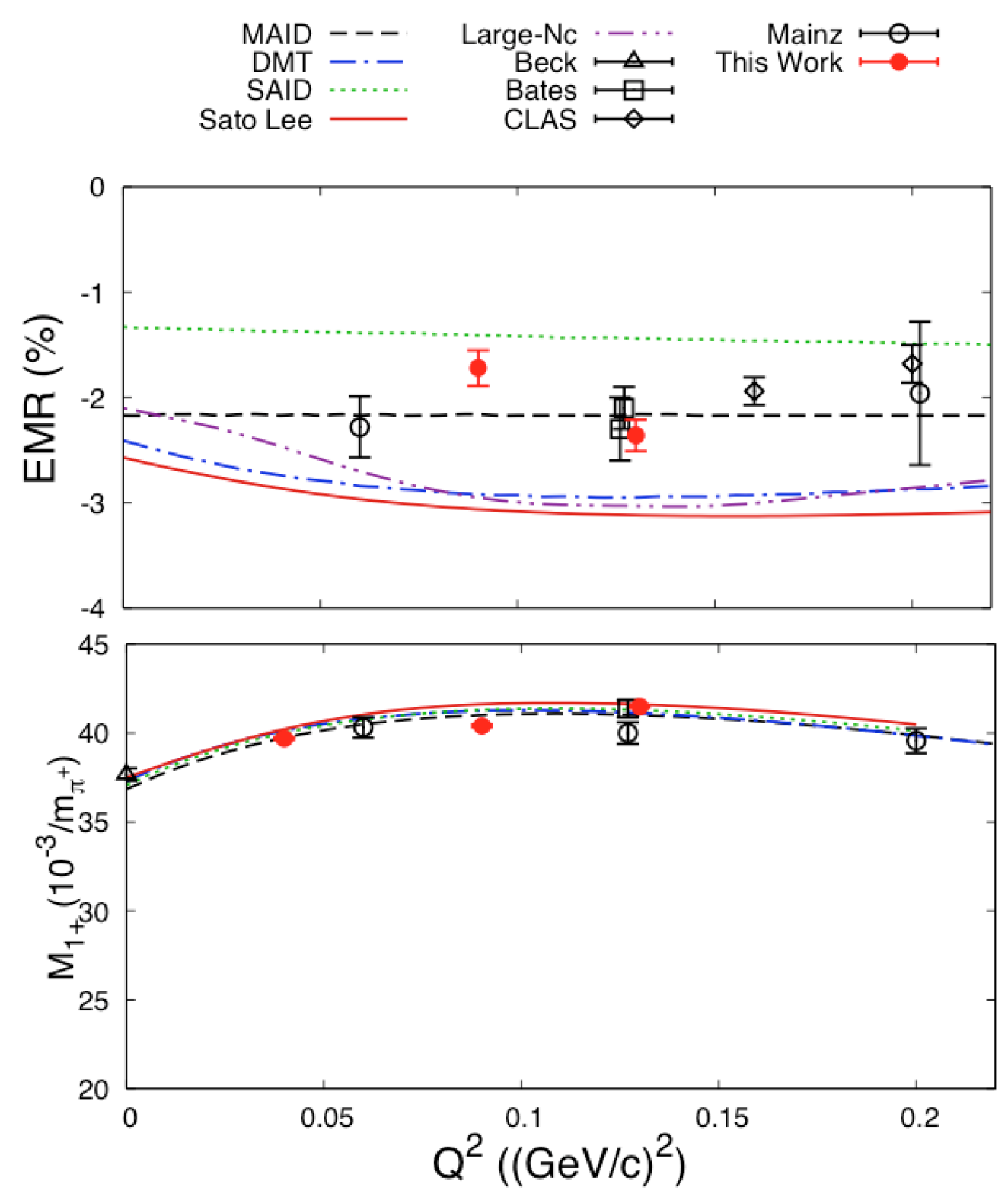

Figure 6.6: $\mathrm{Q}^{2}$ evolution of the $M_{1+}^{3 / 2}$ and $E_{1+}^{3 / 2}$ multipoles. Data from the current work and [11, $22,24,37,39]$ are shown with their statistical and systematic uncertainties added in quadrature. Theoretical predictions from MAID [32, 33], DMT [31], SAID [35], Sato-Lee [30], and the large-Nc calculation [77] are also shown. 
The success of the phenomenological Sato Lee model suggests separation of nucleon dynamics into contributions from the pion cloud and quark-gluon core. Bare (with no pion cloud contributions) Sato Lee model predictions suggest the EMR and CMR should converge as $Q^{2} \rightarrow 0$ due to the long-wave limit. This data shows a trend towards this limit for the first time. The convergence of the CMR and EMR ratios at $Q^{2}=0$ suggests that the meson cloud contribution to both quadrupole amplitudes is similar as we enter the low $Q^{2}$ regime. The bare Sato Lee model also provides a mechanism for comparison of the quark-gluon core dynamics with other models without pion cloud dynamics. In particular, the bare Sato Lee model is qualitatively similar to the prediction of a model based on the Dyson-Schwinger Equation of QCD [78] (DSEM). The agreement between the data and the Sato Lee prediction suggest a possible link between the bare quark-core of a dynamical model and the genuine QCD dynamics.

Despite the success of the Large $\mathrm{N}_{c}$ model in predicting the CMR, this model is known to have issues predicting the two multipoles independently [77]. The constituent quark model from Capstick [8] and the hypercentral quark model (HQM) [49] both fail to describe the data, demonstrating that the color hyperfine models can not explain the dynamics at large distances. Chiral effective field theoretical calculations [47, 48] show promise in a qualitative discussion of the CMR, but are not quite there in magnitude yet. Chiral perturbation theory offers the natural framework to investigate the role of pionic contributions to the nucleon structure through pion loops (the pion cloud) but such contributions are, in general, not scale-independent [79] and thus can not provide a model independent definition. Lattice QCD models [27] have not historically tracked data well. Ongoing Lattice QCD calculations with lighter quark masses of $180 \mathrm{MeV}$ will provide results at lower $Q^{2}$, with reduced uncertainties [80]. These calculations so far indicate [80] that the discrepancy between Lattice QCD and the data gets smaller as the pion mass approaches the physical value, and results calculated with physical masses are expected soon. These data will provide a strong benchmark to measure the new predictions against. 


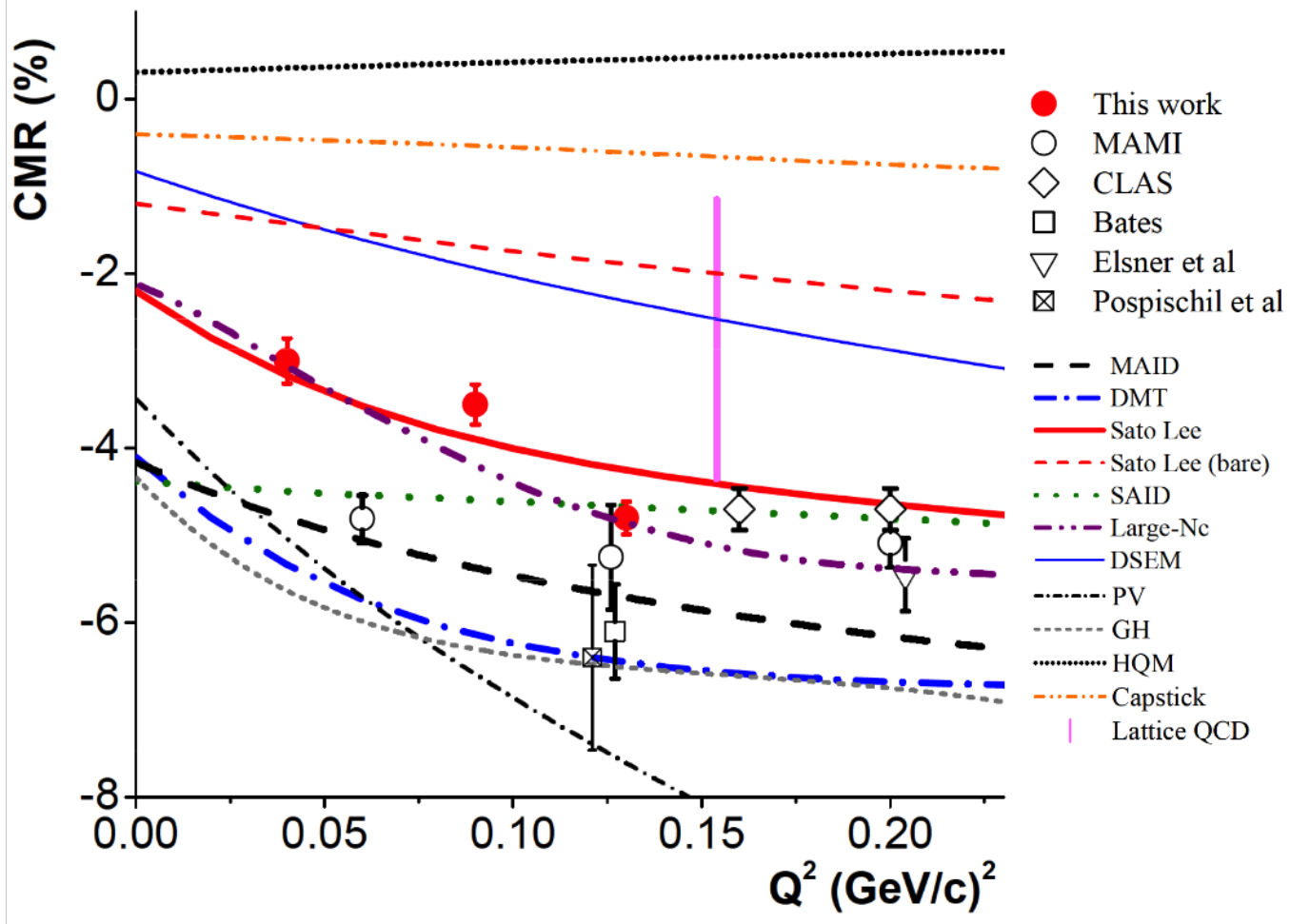

Figure 6.7: $\mathrm{Q}^{2}$ evolution of the $\mathrm{CMR}\left(S_{1+}^{3 / 2} / M_{1+}^{3 / 2}\right)$. Data from the current work, and $[13,22,24,36,37,39]$ are shown with their statistical and systematic uncertainties added in quadrature. The theoretical predictions of MAID [32, 33], DMT [31], SAID [35], Sato-Lee [30], Capstick [8], HQM [49], the Lattice-QCD calculation [27], the large-Nc calculation [77], the DSEM [78], the ChEFT of Pascalutsa-Vanderhaegen (PV) [47] and the Gail-Hemmert (GH) [48] are also shown. 


\subsection{MAMI}

Data were collected in four kinematic settings during the MAMI A1/03-12 experiment. These were broken into two asymmetry pair measurements at $Q^{2}=0.2(\mathrm{GeV} / \mathrm{c})^{2}$ and $\mathrm{W}=$ $1232 \mathrm{MeV}$. Note the model uncertainty could not be calculated in the same fashion as for the JLab experiment since only one model was used. Repeated extractions with the elastic form factors and tunable parameters used in the model varied, leading to an uncertainty in the extraction of $0.1 \%$, which was absorbed into the systematic uncertainties.

\subsubsection{Cross Sections and Asymmetries}

Spectrometer cross sections for each setting are summarized in Table 6.5, and extracted asymmetries are shown in Table 6.6. Statistical uncertainties in the asymmetry are calculated algebraically from the statistical uncertainties of the spectrometer cross sections. Systematic uncertainties are estimated from the RMS deviation of extractions with each spectrometer cross section varying by its systematic uncertainty independently.

Figure 6.8 shows the asymmetry measured at MAMI with DR model predictions for several values of the CMR, calculated by changing only the $S_{1+}^{3 / 2}$ multipole used in the code. The $M_{1+}^{3 / 2}$ multipole is still fixed, and the phase between the imaginary and real parts of the $S_{1+}^{3 / 2}$ multipole are also fixed according to the MAID [32, 33] predictions. The data clearly show a non-zero value for the CMR is appropriate.

Table 6.5: Measured photon electroproduction cross sections from MAMI A1/03-12. Uncertainties are statistical, and systematic.

\begin{tabular}{|c|c|c|c|c|}
\hline$Q^{2}(\mathrm{GeV} / \mathrm{c})^{2}$ & $\mathrm{~W}(\mathrm{MeV})$ & $\theta_{\gamma \gamma}^{*}\left(^{\circ}\right)$ & $\phi_{\gamma \gamma}^{*}\left(^{\circ}\right)$ & $d^{5} \sigma / d k_{\text {elab }}^{\prime} d \Omega_{\text {elab }}^{\prime} d \Omega_{c m}\left(\mathrm{pb} / \mathrm{GeV} / \mathrm{sr}^{2}\right)$ \\
\hline 0.20 & 1232 & 128 & 0 & $497 \pm 11 \pm 13$ \\
\hline 0.20 & 1232 & 128 & 180 & $450 \pm 8 \pm 12$ \\
\hline 0.20 & 1232 & 138 & 0 & $498 \pm 14 \pm 13$ \\
\hline 0.20 & 1232 & 138 & 180 & $412 \pm 12 \pm 11$ \\
\hline
\end{tabular}

Table 6.6: Asymmetries extracted at from VCS cross sections $\mathrm{W}=1232 \mathrm{MeV}$ and $\mathrm{Q}^{2}=$ $0.2(\mathrm{GeV} / \mathrm{c})^{2}$ from MAMI A1/03-12. Uncertainties are statistical, and systematic.

\begin{tabular}{|c|c|}
\hline$\theta_{\gamma \gamma}^{*}\left({ }^{\circ}\right)$ & Asymmetry $(\%)$ \\
\hline 128 & $-5.0 \pm 1.4 \pm 1.1$ \\
\hline 138 & $-9.4 \pm 2.0 \pm 1.1$ \\
\hline
\end{tabular}




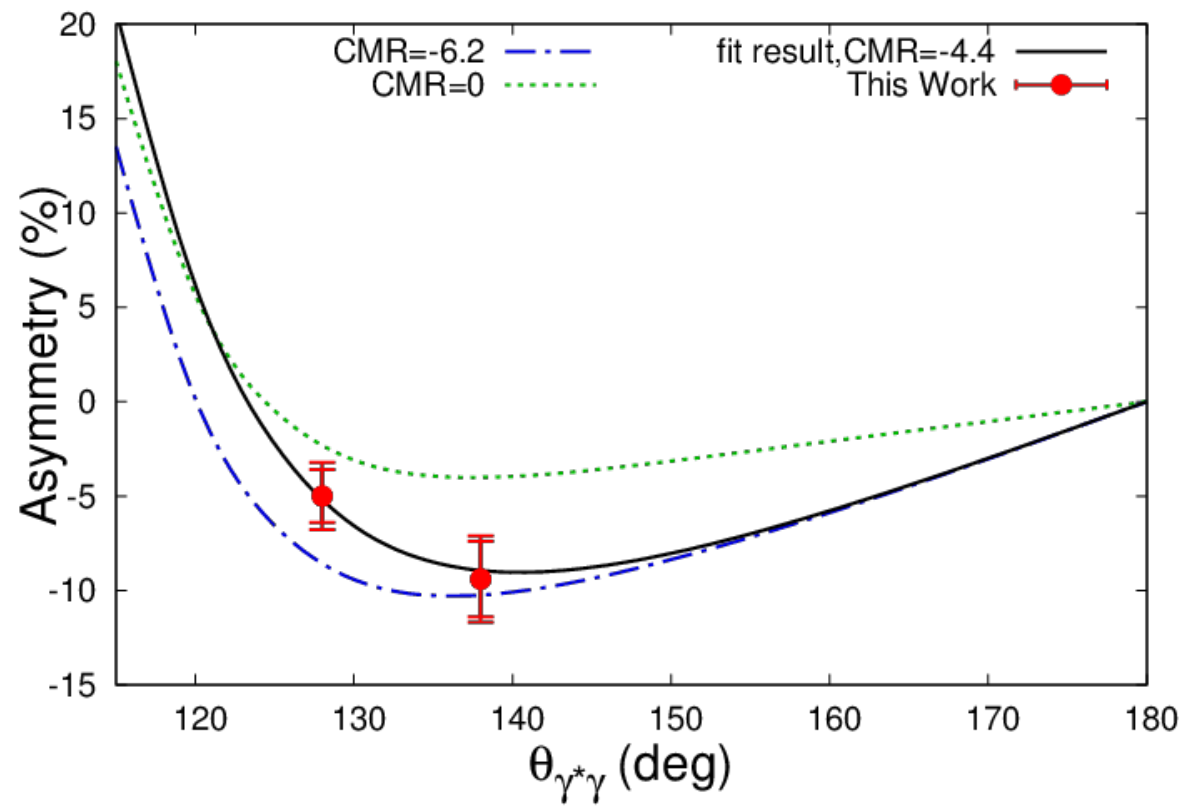

Figure 6.8: Asymmetries extracted at from VCS cross sections $\mathrm{W}=1232 \mathrm{MeV}$ and $\mathrm{Q}^{2}=$ $0.2(\mathrm{GeV} / \mathrm{c})^{2}$ from MAMI A1/03-12. Error bars show statistical uncertainty and statistical plus systematic uncertainty added in quadrature. The lines drawn show the DR model prediction with the MAID $S_{1+}^{3 / 2}$ multipole varied from the standard model prediction (blue dash-dot) to zero (green dash) and the final fit result in solid black. 


\subsubsection{Fitted Extractions}

The CMR and electric generalized polarizability were extracted from the data with the same fitting procedure used in the JLab data utilizing the DR model [60, 61]. Statistical uncertainties are calculated in the Migrad routine of Minuit using the cross section statistical uncertainties in the fit. Systematic uncertainties are estimated through the root mean square (RMS) variation of extractions varying each spectrometer cross section independently by its systematic uncertainty. The results are summarized in Table 6.7 and Figures 6.9 and 6.10 .

Table 6.7: Results extracted at from VCS cross sections $\mathrm{W}=1232 \mathrm{MeV}$ and $\mathrm{Q}^{2}=0.2(\mathrm{GeV} / \mathrm{c})^{2}$ from MAMI A1/03-12. Uncertainties are statistical, and systematic.

\begin{tabular}{|c|c|}
\hline Value & $d^{5} \sigma / d k_{\text {elab }}^{\prime} d \Omega_{\text {elab }}^{\prime} d \Omega_{c m}\left(\mathrm{pb} / \mathrm{GeV} / \mathrm{sr}^{2}\right)$ \\
\hline CMR $(\%)$ & $-4.4 \pm 0.9 \pm 0.6$ \\
\hline$\alpha_{E}\left(10^{-3} \mathrm{fm}^{3}\right)$ & $5.8 \pm 0.6 \pm 1.2$ \\
\hline
\end{tabular}

Figure 6.9 shows the full complement of data addressed in this thesis. The VCS point from MAMI A1/03-12 is in red at $\mathrm{Q}^{2}=0.2(\mathrm{GeV} / \mathrm{c})^{2}$ (furthest right). This point is in good agreement with previous measurements [36, 37, 39] and also in agreement with the Sato Lee model. Our data represents the first VCS channel measurement of the CMR in the low momentum transfer regime, and shows good agreement with the pion channel methods. Since the background terms from VCS are substantially different from those in pion electroproduction, the agreement is strong evidence that the theoretical models used in the extractions are successful in interpreting the signal properly.

The unexplained enhancement in the data compared to models around $\mathrm{Q}^{2}=0.33(\mathrm{GeV} / \mathrm{c})^{2}$ is clearly shown in Figure 6.10. Our data falls between the model predictions, and can not distinguish between the them. More experiments required to fully discern the trend in this region. MAMI A1/03-12 ran as part of a dedicated effort to map this region using VCS both above and below the pion threshold, and results are expected soon. Additionally, the VCS channel can be seen in the JLab E08-010 data discussed in this thesis. VCS in the region $\mathrm{Q}^{2}=0.04-0.13(\mathrm{GeV} / \mathrm{c})^{2}$ may be explored, though with limited statistics. Finally, a proposal has been submitted [57] to Jefferson Laboratory Hall $\mathrm{C}$ to explore the region $\mathrm{Q}^{2}=0.3-0.75(\mathrm{GeV} / \mathrm{c})^{2}$. 


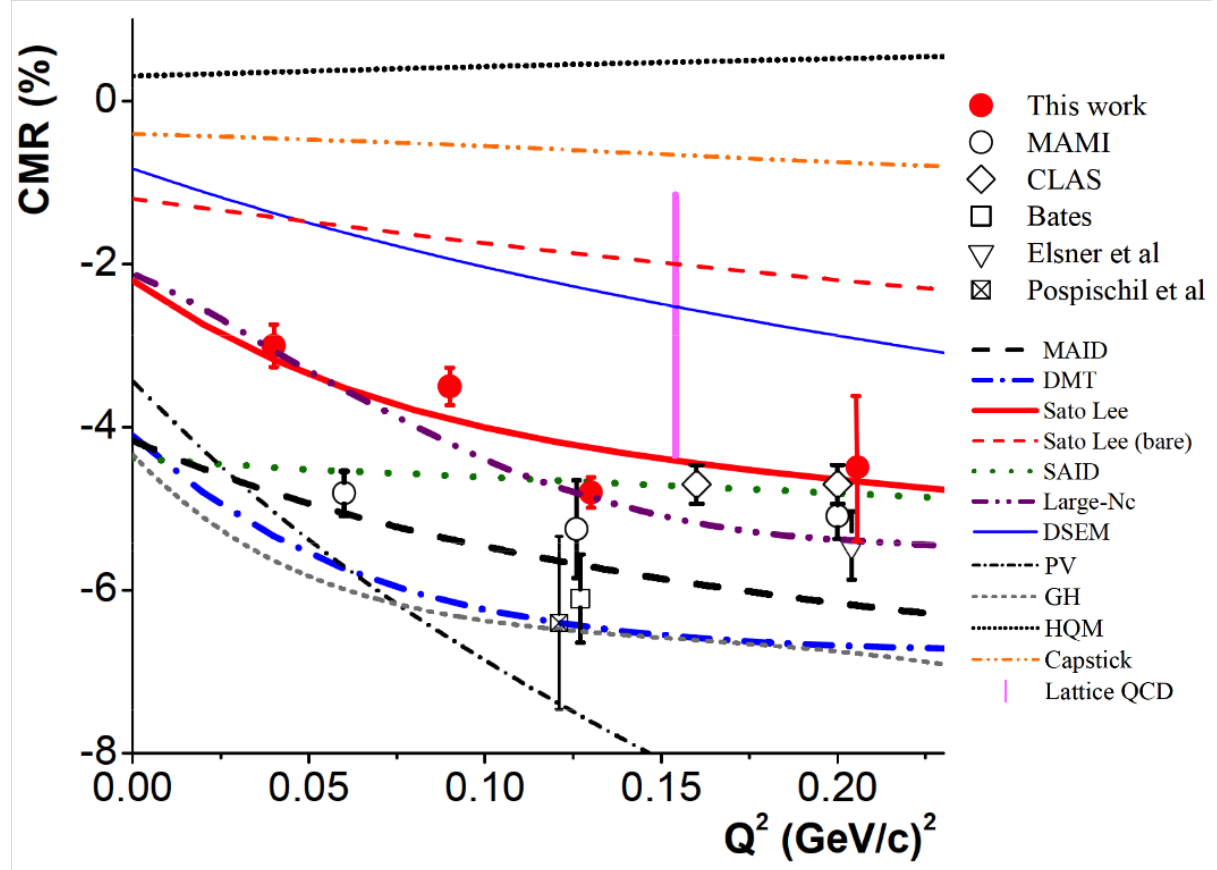

Figure 6.9: $\mathrm{Q}^{2}$ evolution of the $\mathrm{CMR}\left(S_{1+}^{3 / 2} / M_{1+}^{3 / 2}\right)$. Data from the current work, and $[13,22,24,36,37,39]$ are shown with their statistical and systematic uncertainties added in quadrature. The theoretical predictions of MAID [32, 33], DMT [31], SAID [35], Sato-Lee [30], Capstick [8], HQM [49], the Lattice-QCD calculation [27], the large-Nc calculation [77], the DSEM [78], the ChEFT of Pascalutsa-Vanderhaegen (PV) [47] and the Gail-Hemmert (GH) [48] are also shown. 


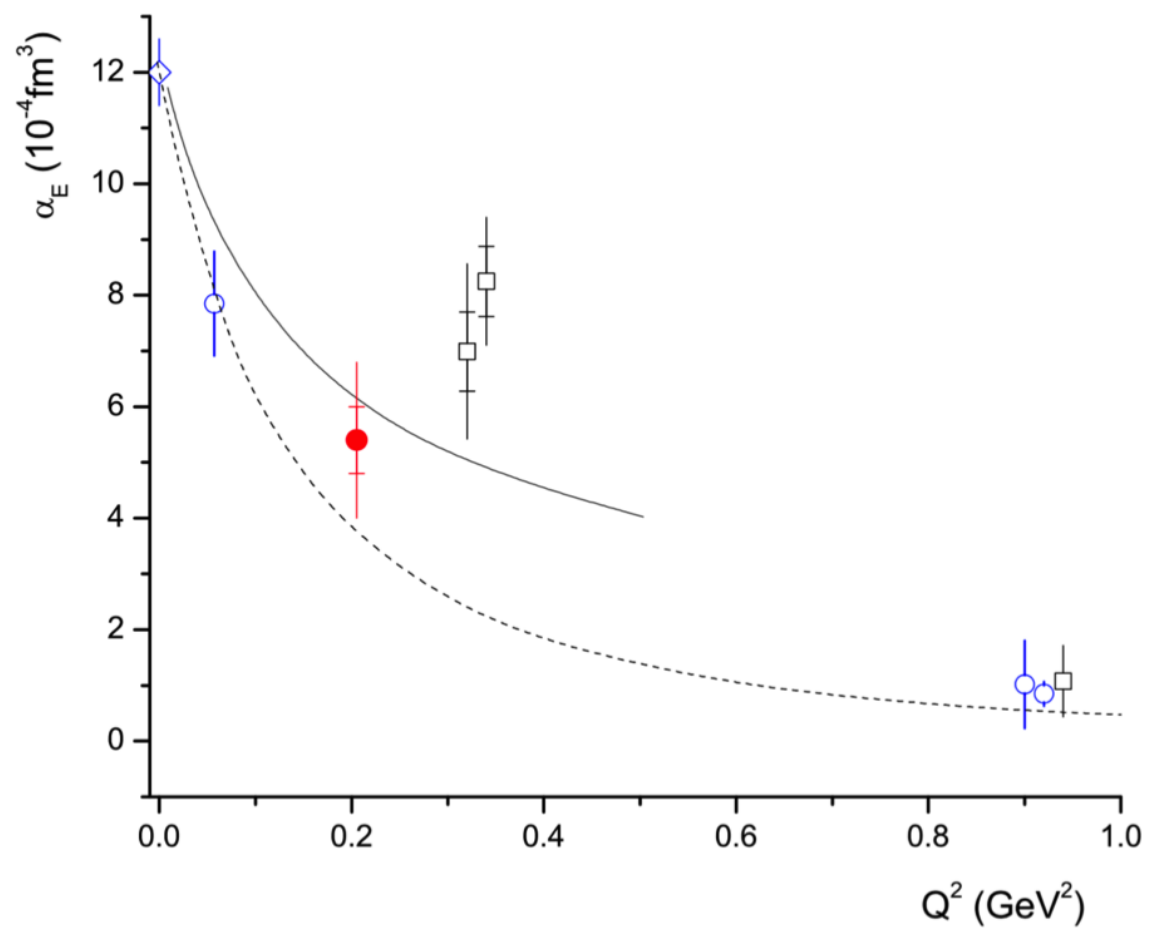

Figure 6.10: Generalized electric polarizability extracted at from VCS cross sections at $\mathrm{W}=$ $1232 \mathrm{MeV}$ and $\mathrm{Q}^{2}=0.2(\mathrm{GeV} / \mathrm{c})^{2}$ from MAMI A1/03-12. World data [51-55] shown with open boxes were extracted with LEX, and open circles from DR. The solid curve corresponds to HBChPT $[81,82]$. The dashed line shows dipole fall off from the DR calculation. 


\section{CHAPTER 7}

\section{CONCLUSIONS}

We discuss pion electroproduction and VCS experiments at the $\Delta$ resonance in the low momentum transfer $\left(Q^{2}=0.04-0.2(\mathrm{GeV} / \mathrm{c})^{2}\right)$ regime. Both experiments discussed utilized kinematic pairs to measure the azimuthal asymmetry and pion electroproduction partial cross sections were extracted. Additionally, direct measurements of the $\mathrm{M}_{1+}^{3 / 2}$ multipole as well as the EMR and CMR are presented. Finally, the electric generalized polarizability was extracted from VCS experiment.

Our data show good agreement with previous results, significantly improving the precision of pion electroproduction data at $\mathrm{Q}^{2}=0.13(\mathrm{GeV} / \mathrm{c})^{2}$, and mapping in high precision the phase space all the way to $\mathrm{Q}^{2}=0.04(\mathrm{GeV} / \mathrm{c})^{2}$. This new mapping has shown dramatic departure from the trend of the data above $\mathrm{Q}^{2}=0.1(\mathrm{GeV} / \mathrm{c})^{2}$. For the first time, our results show the EMR and CMR tend to converge with $\mathrm{Q}^{2} \rightarrow 0$, as predicted by the Sato Lee model [30]. Sato Lee is a dynamical model with quark-gluon core and pion cloud degrees of freedom. The success of the Sato Lee model in describing the data suggests a mechanism to connect with genuine quark core dynamics as predicted by the Dyson-Schwinger Equation of QCD (DSEM) [78]. The full Sato Lee calculation agrees well with data, and the bare model of just the core dynamics shows promising agreement with DSEM. Lattice QCD results are expected soon with lower pion masses [80] around $180 \mathrm{MeV}$. The data presented here will be a key benchmark in assessing the success of new calculations.

Additionally, the CMR was extrapolated from VCS for the first time. This point is in good agreement with high precision measurement via pion electroproduction [38]. The agreement of measurements from two reaction channels with different theoretical underpinnings and background terms suggests the models are able to successfully isolate the detected signal. 
Finally, the electric generalized polarizability was measured in a region where previous data and theoretical models disagree. Our result here can neither confirm nor refute the discrepancy. Other experiments were run at Mainz to attempt to address this issue in detail, and should provide more information soon. Additionally, a new experiment at JLab Hall C has been proposed [57] to map $\mathrm{Q}^{2}=0.33-0.75(\mathrm{GeV} / \mathrm{c})^{2}$.

This work provides key benchmarks to validate theoretical models. Previous results have been verified, and new trends identified. More work is required to fully map the phase space of interest, particularly in the electric GP. 


\section{REFERENCES}

[1] LHCb Collaboration. Phys. Rev. Lett. 115, 072001 (2015).

[2] LHCb Collaboration. Phys. Rev. Lett. 112, 222002 (2014).

[3] A.M. Bernstein, Eur. Phys. J. A 17, 349 (2003).

[4] A.M. Bernstein and C.N. Papanicolas, AIP Conf. Proc. 904, 1 (2007).

[5] A. de Rujula, H. Georgi and S.L. Glashow et al., Phys. Rev D 12, 147 (1975).

[6] S.L. Glashow, Physica A 96, 327 (1979).

[7] N. Isgur, G. Karl and R. Koniuk, Phys. Rev. D 25, 2394 (1982).

[8] S. Capstick and G. Karl, Phys. Rev. D 41, 2767 (1990).

[9] G. Blanpied et al., Phys. Rev. Lett. 79, 4337 (1997).

[10] R. Beck et al., Phys. Rev. Lett. 78, 606 (1997); ibid. 79, 4515 (1997) (Erratum).

[11] R. Beck et al., Phys. Rev. C 61, 035204 (2000).

[12] V.V. Frolov et al., Phys. Rev. Lett. 82, 45 (1999).

[13] T. Pospischil et al., Phys. Rev. Lett. 86, 2959 (2001).

[14] C. Mertz et al., Phys. Rev. Lett. 86, 2963 (2001).

[15] P. Bartsch et al., Phys. Rev. Lett. 88, 142001 (2002).

[16] L.D. van Buuren et al., Phys. Rev. Lett. 89, 012001 (2002).

[17] K. Joo et al., Phys., Rev. Lett. 88, 122001 (2002). 
[18] N.F. Sparveris et al., Phys. Rev. C 67, 058201 (2003).

[19] C. Kunz et al., Phys. Lett. B 564, 21 (2003).

[20] K. Joo et al., Phys. Rev. C 68, 032201 (2003).

[21] K. Joo et al., Phys. Rev. C 70, 042201 (2004).

[22] N.F. Sparveris et al., Phys. Rev. Lett. 94, 022003 (2005).

[23] J.J. Kelly et al., Phys. Rev. Lett. 95, 102001 (2005).

[24] S. Stave et al., Eur. Phys. J. A 30, 471 (2006).

[25] M. Ungaro et al., Phys. Rev. Lett. 97, 112003 (2006).

[26] Z.L. Zhou et al., Nucl. Instrum. Methods A 487, 365 (2002).

[27] C. Alexandrou et al., Phys. Rev. Lett. 94, 021601 (2005).

[28] C. Alexandrou et al., Phys. Rev D 77, 085012 (2008).

[29] C. Alexandrou et al., Phys. Rev D 83, 014501 (2011).

[30] T. Sato and T.-S.H. Lee, Phys. Rev. C 63, 055201 (2001).

[31] S.S. Kamalov and S.N. Yang, Phys. Rev. Lett. 83, 4494 (1999)

[32] S.S. Kamalov et al., Phys. Lett. B 522, 27 (2001).

[33] D. Drechsel et al., Nucl. Phys. A 645, 145 (1999).

[34] D. Drechsel and L. Tiator, J. Phys. G 18, 449 (1992)

[35] R.A. Arndt, et al., Phys. Rev. C 66, 055213 (2002); nucl-th/ 0301068 , http: //gwdac.phys.gwu.edu.

[36] D. Elsner et al., Eur. Phys. J. A 2791 (2006).

[37] N. F. Sparveris et al., Phys. Lett. B 651, 102 (2007).

[38] S. Stave et al., Phys. Rev. C 78, 025209 (2008)

[39] I. G. Aznauryan et al., Phys. Rev. C 80, 055203 (2009) 
[40] A. N. Villano et al., Phys. Rev. C 80, 035203 (2009)

[41] J. Kirkpatrick et al., Phys. Rev. C 84, 028201 (2011).

[42] N. Sparveris et al., Eur. Phys. J. A 49, 136 (2013).

[43] N. Sparveris et al., Phys. Rev. C 78, 018201 (2008).

[44] D.-H. Lu, A. W. Thomas, and A. G. Williams, Phys. Rev. C 55, 3108 (1997).

[45] U. Meyer, E. Hernandez, and A. J. Buchmann, Phys. Rev. C 64, 035203 (2001).

[46] M. Fiolhais, B. Golli, and S. Sirca, Phys. Lett. B 373, 229 (1996).

[47] V. Pascalutsa and M. Vanderhaeghen, Phys. Rev. D 73, 034003 (2006).

[48] T. A. Gail and T. R. Hemmert, Eur. Phys. J. A 28 (1), 91 (2006).

[49] M. De Sanctis et al., Nucl. Phys. A 755, 294 (2005).

[50] J. Mandeville et al., Phys. Rev. Lett. 72, 3325 (1994).

[51] J. Roche, et al., Phys. Rev. Lett. 85, 708 (2000).

[52] P. Janssens, et al., Eur. Phys. J. A 37, 1 (2008).

[53] G. Laveissiere, et al., Phys. Rev. Lett. 93, 122001 (2004).

[54] H. Fonvieille, et al., Phys. Rev. C 86, 015210 (2012).

[55] P. Bourgeois, et al., Phys. Rev. Lett. 97, 212001 (2006).

[56] S. Stave. Ph.D. thesis. Massachusetts Institute of Technology, (2006).

[57] Jefferson Lab proposal PR12-15-001.

[58] D. Anez, Ph.D. thesis, Dalhousie University (2014).

[59] P.A.M. Guichon, G.Q. Liu and A.W. Thomas, Nucl. Phys. A 591, 606 (1995).

[60] B. Pasquini, M. Gorchtein, D. Drechsel, A. Metz, M. Vanderhaeghen, Eur. Phys. J. A 11, 185 (2001).

[61] D. Drechsel, B. Pasquini, M. Vanderhaeghen, Phys. Rept. 378, 99 (2003). 
[62] E. Stiliaris and C. N. Papanicolas, nucl-ex/0703031, (2007).

[63] LHC Computing Grid. Minuit Website, http://seal.web.cern.ch/seal/ snapshot/work-packages/mathlibs/minuit/.

[64] C. W. Leemann, D.R. Douglas, and G.A. Krafft, Annu. Rev.Nucl. Part. Sci. 51 (2001) 413.

[65] J. Alcorn et al., Nucl. Instrum. Methods A 522, 294 (2004).

[66] R. Michaels, et al., Jefferson Lab HALL A Logbook - Full Listing, http:// hallaweb.jlab.org/halog/log/html/logdir.html.

[67] J. Dahlberg, Jefferson Lab Alignment Group Data Transmittal, (2011), https: / / www.jlab.org/accel/survalign/documents/dthalla/A1379.pdf.

[68] B1 Collaboration. Mainz MAMI Website, http://kph.uni-mainz . de/B1/.

[69] W. U. Boeglin. Czech. J. Phys. 45, 295 (1995).

[70] A. Jankowiak, et. al., Proc. EPAC, Paris, France, 1085 (2002).

[71] K. I. Blomqvist, et al., Nucl. Instrum. Methods A 403, 263 (1998).

[72] S. Sirca. Ph.D. thesis. University of Ljubljana, (1999).

[73] A1 Collaboration. Mainz Al Website, http://wwwa1.kph.uni-mainz.de/ A1/.

[74] J. Mougey, E89-044 Analysis Progress Report. Jefferson Lab, (2000).

[75] M. Mazouz, P. Y. Bertin, and E. Voutier, Tech. Rep. Jefferson Lab, (2005), JLABTN05-42.

[76] MCEEP: Monte Carlo for (e,e'p) experiments, http://hallaweb.jlab.org/ software/mceep/mceep.html.

[77] V. Pascalutsa and M. Vanderhaeghen, Phys. Rev. D 76, 111501 (2007).

[78] J. Segovia, I. C. Clot, Craig D. Roberts and Sebastian M. Schmidt, Few Body Syst. 55 (2014) 1185 
[79] U. Meissner, AIP. Conf. Proc. 904, 142 (2007), nucl-th/ 0701094.

[80] C. Alexandrou, private communication, to be published.

[81] T. R. Hemmert, B. R. Holstein, G. Knochlein, S. Scherer, Phys. Rev. Lett. 79, 22 (1997).

[82] T. R. Hemmert, B. R. Holstein, G. Knochlein, D. Drechsel, Phys. Rev. D 62, 014013 (2000). 